\title{
Organocatalyzed Enantioselective Direct Mannich Reaction of $\alpha$-Styrylacetates
}

Jie Guang, Saroj Rout, Manisha Bihani, Ariel J. Larson,

\author{
Hadi D. Arman, and John C.-G. Zhao* \\ Department of Chemistry \\ University of Texas at San Antonio

\section{Supporting Information}

\section{Table of Contents}

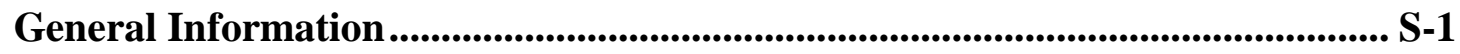

Experimental Procedures ................................................................................. S-1

Proposed Transition States............................................................................................ S-3

Compound Characterization Data ...................................................................... S-5

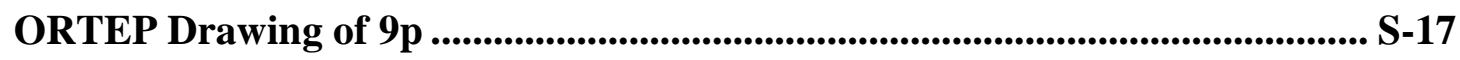

Copy of NMR Spectra .................................................................................... S-18

Copy of HPLC Chromatograms............................................................................... S-38 


\section{General Information}

Unless otherwise noted, ${ }^{1} \mathrm{H}$, and ${ }^{13} \mathrm{C}$ NMR spectra were obtained on a $500 \mathrm{MHz}$ spectrometer using the residual solvent as the standard. The following abbreviations were used to designate chemical shift mutiplicities: $\mathrm{s}=$ singlet, $\mathrm{d}=$ doublet, $\mathrm{t}=$ triplet, $\mathrm{q}=$ quartet, $\mathrm{h}=$ heptet, $\mathrm{m}=$ multiplet, $\mathrm{br}=$ broad . All first-order splitting patterns were assigned on the basis of the appearance of the multiplet. Splitting patterns that could not be easily interpreted are designated as multiplet (m) or broad (br). Optical rotations were recorded on an Autopol IV automatic polarimeter. Melting points were recorded on MEL-TEMP melting point apparatus in open capillaries and uncorrected. TLC was performed with silica gel GF254 precoated on aluminum plates (Dynamic Adsorbents, Inc), and spots were visualized with UV. Flash column chromatography was performed on silica gel. HPLC analysis was performed on a Shimadzu instrument with LC-10AT pump and SPD-10AV UV-Vis detector. ChiralPak IC HPLC column was purchased from Daicel Chemical Industry, Ltd. HRMS were conducted by the Department of Chemistry, Louisiana State University or the RCMI core facilities at UTSA. Compounds used in this study were purchased from Aldrich, AK Scientific, or Fisher and were used as received.

\section{Experimental Procedures}

\section{Representative Experimental Procedure for the Mannich Reaction}

The solution of catalyst 7c $(3.5 \mathrm{mg}, 0.0050 \mathrm{mmol}, 5.0 \mathrm{mmol} \%), N$-tosyl imine 8a (25.9 $\mathrm{mg}, 0.10 \mathrm{mmol})$, and ester $\mathbf{5 d}(60.4 \mathrm{mg}, 0.20 \mathrm{mmol})$ in toluene $(0.5 \mathrm{~mL})$ was stirred under nitrogen at room temperature for 3 days (monitored by TLC). Upon the completion of the reaction, the volatile components were removed under reduced pressure. The residue was purified by column chromatography on silica gel with an eluent (20:20:1 hexanes/ $\left.\mathrm{CH}_{2} \mathrm{Cl}_{2} / \mathrm{EtOAc}\right)$ to afford the desired Mannich adduct 9d 


\section{Tosyl Group Removal}<smiles>Cc1ccc2ccccc2c1OC(=O)C(=Cc1ccccc1)[C@H](NC(=O)c1ccccc1)c1cccc(C(F)(F)F)c1</smiles>

To a solution of the compound $9 \mathbf{d}(112.2 \mathrm{mg}, 0.20 \mathrm{mmol})$, 4-(dimethylamino)pyridine (4.5 mg, $0.040 \mathrm{mmol})$, and benzoyl chloride $(112.5 \mathrm{mg}, 0.80 \mathrm{mmol})$ in THF (4.0 mL) was added $\mathrm{Et}_{3} \mathrm{~N}(81.0 \mathrm{mg}, 0.80 \mathrm{mmol})$. The mixture was stirred for $24 \mathrm{~h}$ at $\mathrm{rt}$. Then the solvent was removed under reduced pressure and the residue was directly transferred to a silica gel column and purified by column chromatography (6:1 hexane/AcOEt) to afford the product $\mathbf{1 0}$ as a colorless oil (121.0 $\mathrm{mg}, 91 \%)$.

To a mixture of the compound $10(66.5 \mathrm{mg}, 0.10 \mathrm{mmol})$ and HMPA $(0.2 \mathrm{~mL})$ in THF $(2.0 \mathrm{~mL})$ under argon at $-78{ }^{\circ} \mathrm{C}$ was added $\mathrm{SmI}_{2}(4.0 \mathrm{~mL}, 0.10 \mathrm{M}$ solution in THF, $0.40 \mathrm{mmol}$ ). The mixture was then stirred at $-78{ }^{\circ} \mathrm{C}$ for $0.5 \mathrm{~h}$. Then the reaction mixture was then quenched by adding aq. $\mathrm{NaHCO}_{3}$ solution $(10 \mathrm{~mL})$ and extracted with AcOEt $(2 \times 10 \mathrm{~mL})$. The combined organic phase was dried over $\mathrm{Na}_{2} \mathrm{SO}_{4}$ and the solvent was then removed under reduced pressure. The resulting crude mixture was purified by column chromatography (5:1 hexane/AcOEt) to afford the product 11 (43.4 mg, 87\%).

\section{Synthesis of Tetrahydrofuran Derivative 13}

\section{Synthesis of $\gamma$-Aminol 12}


A solution of the purified 9j (97\% ee, >99:1 dr, $94.3 \mathrm{mg}, 0.15 \mathrm{mmol})$ in THF (10.0

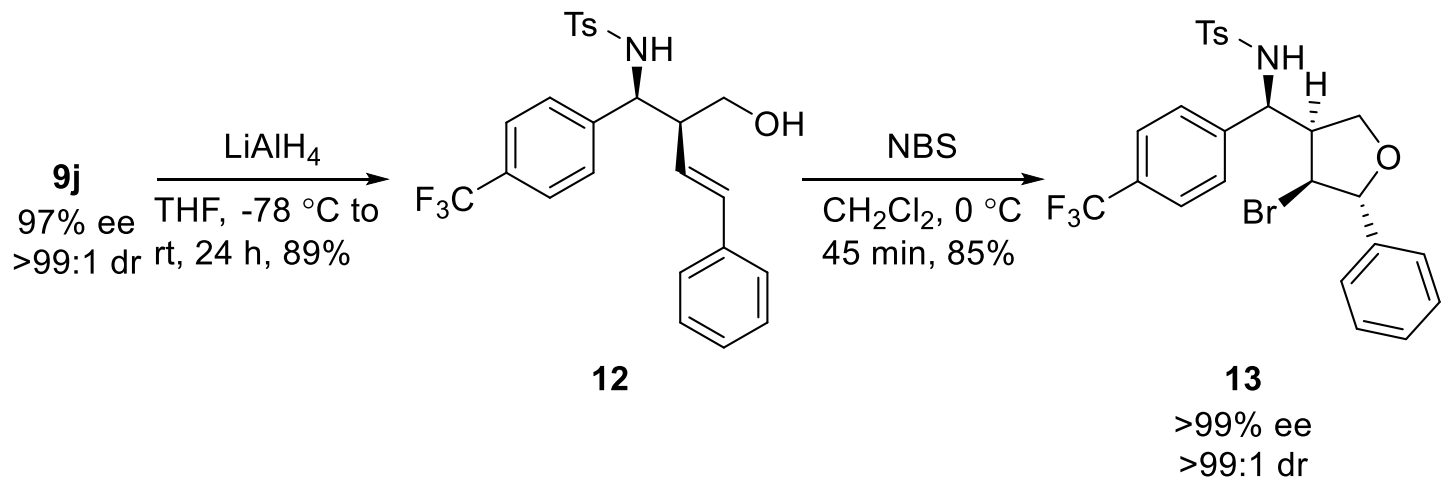

mL) was added to a suspension of $\mathrm{LiAlH}_{4}(16.8 \mathrm{mg}, 0.45 \mathrm{mmol})$ at $-78{ }^{\circ} \mathrm{C}$ under nitrogen. The resulting mixture was then allowed to come to room temperature and further stirred for $24 \mathrm{~h}$. Upon the completion of the reaction (monitored by TLC), the reaction mixture was quenched by adding aq. $\mathrm{NaOH}$ solution $(1 \mathrm{M}, 0.5 \mathrm{~mL})$ and extracted with AcOEt $(2 \times 10 \mathrm{~mL})$. The combined organic phase was dried over $\mathrm{Na}_{2} \mathrm{SO}_{4}$, filtered, and concentrated under reduced pressure. The resulting crude mixture was purified by column chromatography ( $7: 3$ hexane/AcOEt) to afford pure $\gamma$-aminol derivative 12 (63.4 $\mathrm{mg}, 89 \%$ yield).

\section{Synthesis of Tetrahydrofuran Derivative 13}

To a solution of $12(47.5 \mathrm{mg}, 0.1 \mathrm{mmol})$ in $\mathrm{CH}_{2} \mathrm{Cl}_{2}(5.0 \mathrm{~mL})$ at $0{ }^{\circ} \mathrm{C}$ under nitrogen was added NBS (26.5 $\mathrm{mg}, 0.15 \mathrm{mmol})$. The resulting mixture was warmed to room temperature and stirred for $45 \mathrm{~min}$. Upon the completion of the reaction (monitored by TLC), the reaction mixture was extracted with $\mathrm{CH}_{2} \mathrm{Cl}_{2}(15 \mathrm{~mL} \times 2)$ and the combined extracts were washed with water $(10 \mathrm{~mL})$. The organic phase was dried over $\mathrm{Na}_{2} \mathrm{SO}_{4}$, filtered, and concentrated under reduced pressure. The resulting crude mixture was purified by column chromatography (7:3 hexane/AcOEt) to afford the pure tetrahydrofuran derivative $\mathbf{1 3}$ (47 $\mathrm{mg}, 85 \%$ yield).

\section{Proposed Transition States}


On the basis of absolute stereochemistry of the reaction product, plausible transition states were proposed to account for the formation of the major enantiomer in this reaction (Scheme S-1). As shown in Scheme 1, the $\alpha$-styrylacetate is deprotonated by the catalyst to form an enolate. The urea moiety of the catalyst should facilitate the deprotonation through the proximity effects ${ }^{12}$ by forming hydrogen bonds with the ester. After deprotonation, the ester enolate is closely associated with catalyst through ionic interactions. The $R e$ face orientation of this enolate (top equation) is favored over the Si face orientation (bottom equation) because there are less steric interactions between the enolate and the catalyst backbone. On the other hand, the imine forms hydrogen bonds with the urea moiety of the catalyst. These hydrogen bonds not only help fix the orientation of the imine, but also activate the imine for the Mannich reaction. For the orientation of the imine, the $R e$ face (top equation) is also favored due to stronger hydrogen-boding and less steric interference between the tolyl group on the imine and the catalyst. In the favored transition state, the attack of the enolate from back onto the $S i$ face of the imine leads to the formation of the observed $(2 S, 3 S)$-product.

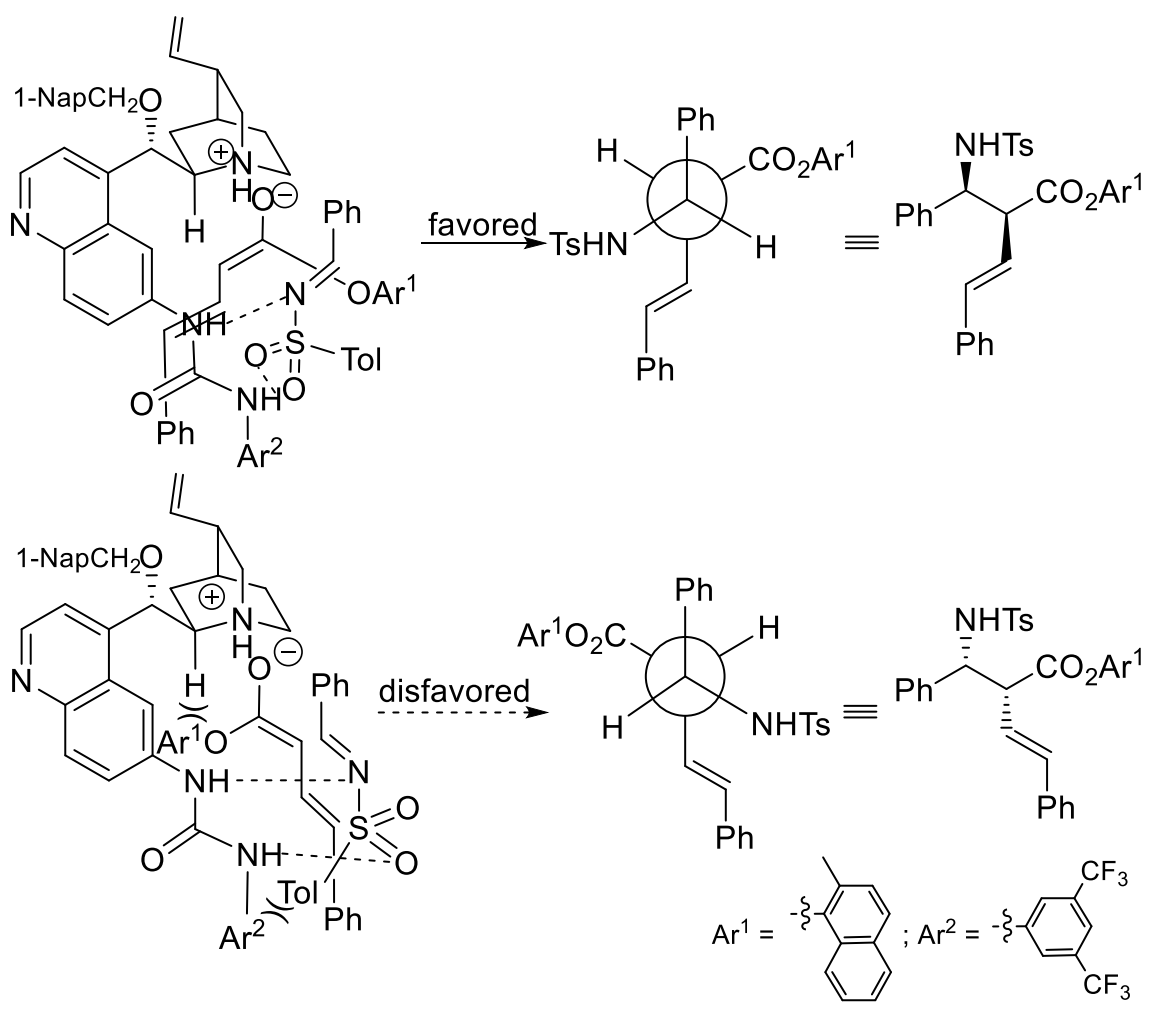

Scheme S-1. Proposed transition states for the ester Mannich reaction 


\section{Compound Characterization Data}

\section{2-Methylnaphthalen-1-yl}

$(S, E)-2-\{(S)-[(4-m e t h y l p h e n y l)$ sulfonamido](phenyl)methyl $\}-4-p h e n y l b u t-3-e n o-$ ate (9d)<smiles>Cc1ccc2ccccc2c1OC(=O)C(/C=C/c1ccccc1)[C@H](NC(F)(F)F)c1ccccc1</smiles>
White solid; $47.6 \mathrm{mg}, 85 \%$ yield m.p. $244-246{ }^{\circ} \mathrm{C}[\alpha]_{\mathrm{D}}{ }^{25}$ $=-168.2$ (c 1.05, THF, 85:15 dr, 90\% ee); ${ }^{1} \mathrm{H}(500 \mathrm{MHz}$, $\left.\left(\mathrm{CD}_{3}\right)_{2} \mathrm{SO}\right): \delta 1.85(\mathrm{~s}, 3 \mathrm{H}), 2.23(\mathrm{~s}, 3 \mathrm{H}), 4.19(\mathrm{t}, J=10.0$ $\mathrm{Hz}, 1 \mathrm{H}), 4.80(\mathrm{t}, J=10.5 \mathrm{~Hz}, 1 \mathrm{H}), 6.24(\mathrm{dd}, J=6.5$, $15.5 \mathrm{~Hz}, 1 \mathrm{H}), 6.89(\mathrm{~d}, J=16.0 \mathrm{~Hz}, 1 \mathrm{H}), 7.04(\mathrm{~d}, \mathrm{~J}=8.0$ $\mathrm{Hz}, 2 \mathrm{H}), 7.10(\mathrm{t}, J=7.5 \mathrm{~Hz}, 1 \mathrm{H}), 7.26-7.46(\mathrm{~m}, 13 \mathrm{H}), 7.67(\mathrm{~d}, J=8.5 \mathrm{~Hz}, 1 \mathrm{H})$, $7.82(\mathrm{~d}, J=8.0 \mathrm{~Hz}, 1 \mathrm{H}) ;{ }^{13} \mathrm{C}\left(125 \mathrm{MHz},\left(\mathrm{CD}_{3}\right)_{2} \mathrm{SO}\right): \delta 14.9,20.6,55.4,58.8,119.8$, $124.4,125.2,125.4,125.9,126.1,127.2,127.4,127.5,127.7,128.0,128.3,128.4$, $128.7,132.2,135.1,135.9,138.4,139.4,141.7,143.0,168.7$; $v_{\max }: 1085,1149,1334$, 1447, 1494, 1600, 1732, $3321 \mathrm{~cm}^{-1}$; Enantiomeric excess of 9d was determined by chiral stationary phase HPLC analysis using a ChiralPak IC column (80:20 hexanes $/ i-\mathrm{PrOH}$ at $1.0 \mathrm{~mL} / \mathrm{min}$ ), major isomer: $\mathrm{t}_{\mathrm{R}}=19.1 \mathrm{~min}$, minor isomer: $\mathrm{t}_{\mathrm{R}}=23.7$ min; HRMS $(\mathrm{m} / z)$ : calcd for $\mathrm{C}_{35} \mathrm{H}_{32} \mathrm{NO}_{4} \mathrm{~S}\left([\mathrm{M}+\mathrm{Na}]^{+}\right)$: 584.1866; found: 584.1859 .

\section{2-Methylnaphthalen-1-yl}

$(S, E)-2-\{(S)-[(4-m e t h y l p h e n y l)$ sulfonamido](p-tolyl)methyl $\}-4-p h e n y l b u t-3-e n o-$ ate $(9 e)$<smiles>Cc1ccc(C(N[As])C(/C=C/c2ccccc2)C(=O)Oc2c(C)ccc3ccccc23)cc1</smiles>

White solid; $38.7 \mathrm{mg}, 67 \%$ yield m.p. $237-239{ }^{\circ} \mathrm{C}$ $[\alpha]_{\mathrm{D}}^{25}=-148.0(\mathrm{c} 1.0, \mathrm{THF}, 86: 14 \mathrm{dr}, 87 \%$ ee $) ;{ }^{1} \mathrm{H}$ $\left(500 \mathrm{MHz},\left(\mathrm{CD}_{3}\right)_{2} \mathrm{SO}\right): \delta 1.97(\mathrm{~s}, 3 \mathrm{H}), 2.23(\mathrm{~s}, 6 \mathrm{H})$, $4.13(\mathrm{t}, J=10.0 \mathrm{~Hz}, 1 \mathrm{H}), 4.69(\mathrm{t}, J=10.5 \mathrm{~Hz}, 1 \mathrm{H})$, $6.21(\mathrm{t}, J=15.0 \mathrm{~Hz}, 1 \mathrm{H}), 6.89(\mathrm{~d}, J=16.0 \mathrm{~Hz}, 1 \mathrm{H})$, 7.01-7.05 (m, 2H), $7.14(\mathrm{t}, J=8.0 \mathrm{~Hz}, 2 \mathrm{H}), 7.27-7.40(\mathrm{~m}, 12 \mathrm{H}), 7.67(\mathrm{~d}, J=8.0 \mathrm{~Hz}$, $1 \mathrm{H}), 7.82(\mathrm{~d}, J=8.0 \mathrm{~Hz}, 1 \mathrm{H}), 8.47(\mathrm{~d}, J=7.5 \mathrm{~Hz}, 1 \mathrm{H}) ;{ }^{13} \mathrm{C}\left(125 \mathrm{MHz},\left(\mathrm{CD}_{3}\right)_{2} \mathrm{SO}\right): \delta$ 
20.6, 21.2, 21.3, 56.2, 59.3, 120.6, 125.1, 125.9, 126.2, 126.6, 126.9, 128.0, 128.2, $128.5,129.1,129.2,129.3,129.5,132.9,133.5,136.6,136.9,137.5,139.0,142.4$, 143.7, 169.9; $v_{\max }: 1070,1145,1330,1442,1493,1598,1731,3317 \mathrm{~cm}^{-1}$; Enantiomeric excess of 9e determined by chiral stationary phase HPLC analysis using a ChiralPak IC column (80:20 hexanes $/ i-\mathrm{PrOH}$ at $1.0 \mathrm{~mL} / \mathrm{min})$, major isomer: $\mathrm{t}_{\mathrm{R}}=$ 27.4 min, minor isomer: $t_{R}=20.8 \mathrm{~min} ; \operatorname{HRMS}(\mathrm{m} / \mathrm{z})$ calcd for $\mathrm{C}_{36} \mathrm{H}_{33} \mathrm{NNaO}_{4} \mathrm{~S}$ $\left([\mathrm{M}+\mathrm{Na}]^{+}\right): 598.2023$; found: 598.2032.

\section{2-Methylnaphthalen-1-yl}

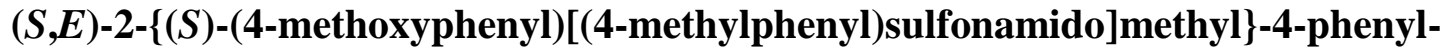
but-3-enoate (9f)<smiles>[CH2]NC(c1ccc(OC)cc1)[C@H](/C=C/c1ccccc1)C(=O)Oc1c(C)ccc2ccccc12</smiles>

White solid; 75\% yield; m.p. 238-240 ${ }^{\circ} \mathrm{C} ;{ }^{1} \mathrm{H}$ NMR $\left(500 \mathrm{MHz},\left(\mathrm{CD}_{3}\right)_{2} \mathrm{SO}\right): \delta 1.91(\mathrm{~s}, 3 \mathrm{H}), 2.23(\mathrm{~s}, 3 \mathrm{H})$, $3.76(\mathrm{~s}, 3 \mathrm{H}), 4.11(\mathrm{t}, J=9.8 \mathrm{~Hz}, 1 \mathrm{H}), 4.68(\mathrm{~s}, 1 \mathrm{H})$, $6.21(\mathrm{t}, J=11 \mathrm{~Hz}, 1 \mathrm{H}), 6.80(\mathrm{~d}, J=7.2 \mathrm{~Hz}, 2 \mathrm{H})$, $6.88(\mathrm{~d}, J=15.7 \mathrm{~Hz}, 1 \mathrm{H}), 7.03(\mathrm{~d}, J=7.4 \mathrm{~Hz}, 3 \mathrm{H})$, $7.19(\mathrm{~d}, J=7.3 \mathrm{~Hz}, 2 \mathrm{H}), 7.48-7.23(\mathrm{~m}, 9 \mathrm{H}), 7.67(\mathrm{~d}, J=8.3 \mathrm{~Hz}, 1 \mathrm{H}), 7.82(\mathrm{~d}, J=$ $7.8 \mathrm{~Hz}, 1 \mathrm{H}), 8.46(\mathrm{~d}, J=9.2 \mathrm{~Hz}, 1 \mathrm{H}) ;{ }^{13} \mathrm{C}\left(125 \mathrm{MHz},\left(\mathrm{CD}_{3}\right)_{2} \mathrm{SO}\right): \delta$ 14.9, 20.5, 55.0, 55.6, 58.3, 113.5, 114.7, 119.9, 124.6, 125.2, 125.4, 125.9, 126.1, 127.2, 127.7, 128.0, $128.3,128.4,128.7,131.4,132.2,134.9,135.9,138.5,139.4,141.6,143.0,158.7$, $168.8 ; v_{\max }: 1029,1073,1148,1251,1330,1447,1514,1611,1730,3316 \mathrm{~cm}^{-1}$; Enantiomeric excess of 9f was determined by chiral stationary phase HPLC analysis using a ChiralPak IC column (80:20 hexanes $/ i-\mathrm{PrOH}$ at $1.0 \mathrm{~mL} / \mathrm{min})$, major isomer: $\mathrm{t}_{\mathrm{R}}$ $=38.3 \mathrm{~min}$, minor isomer: $\mathrm{t}_{\mathrm{R}}=31.0 \mathrm{~min} ; \mathrm{HRMS}(\mathrm{m} / \mathrm{z})$ calcd for $\mathrm{C}_{36} \mathrm{H}_{33} \mathrm{NNaO}_{5} \mathrm{~S}$ $\left([\mathrm{M}+\mathrm{Na}]^{+}\right): 614.1972$; found: 614.1974 .

\section{2-Methylnaphthalen-1-yl}

$(S, E)-2-\{(S)$-(4-fluorophenyl)[(4-methylphenyl)sulfonamido]methyl\}-4-phenylbut

\section{-3-enoate $(9 \mathrm{~g})$}

White solid; $45.7 \mathrm{mg}, 79 \%$ yield; m.p. $259-261^{\circ} \mathrm{C}[\alpha]_{\mathrm{D}}{ }^{25}=-178.0(\mathrm{c} 0.95$, THF, 88:12 
dr, 86\% ee); ${ }^{1} \mathrm{H}$ NMR (500 MHz, $\left.\left(\mathrm{CD}_{3}\right)_{2} \mathrm{SO}\right): \delta 1.90$ (s, 3H), $2.23(\mathrm{~s}, 3 \mathrm{H}), 4.13$ (t, $J=9.8$<smiles>Cc1ccc2ccccc2c1OC(=O)[C@H](/C=C/c1ccccc1)[C@H](NC(F)(F)F)c1ccc(F)cc1</smiles>
$\mathrm{Hz}, 1 \mathrm{H}), 4.76(\mathrm{~s}, 1 \mathrm{H}), 6.24(\mathrm{dd}, J=15.4,9.1 \mathrm{~Hz}, 1 \mathrm{H})$, $6.87(\mathrm{~d}, J=15.8 \mathrm{~Hz}, 1 \mathrm{H}), 7.06(\mathrm{dd}, J=16.5,8.1 \mathrm{~Hz}$, $5 \mathrm{H}), 7.31(\mathrm{dd}, J=13.2,5.7 \mathrm{~Hz}, 4 \mathrm{H}), 7.41(\mathrm{q}, J=7.3$ $\mathrm{Hz}, 7 \mathrm{H}), 7.69$ (d, $J=8.4 \mathrm{~Hz}, 1 \mathrm{H}), 7.82$ (t, $J=13.8 \mathrm{~Hz}$, $1 \mathrm{H}), 8.58(\mathrm{~s}, 1 \mathrm{H}) ;{ }^{13} \mathrm{C} \mathrm{NMR}\left(125 \mathrm{MHz},\left(\mathrm{CD}_{3}\right)_{2} \mathrm{SO}\right): \delta$ 15.7, 21.6, 56.2, 58.7, 115.4, 115.6, 120.3, 126, 126.3, 126.6, 126.7, 126.9, 128.1, $128.6,129.1,129.2,129.5,130.3,130.4,132.9,132.9,136.5,138.8,142.6,143.6$, 169.8; Enantiomeric excess of $\mathbf{9 g}$ was determined by chiral stationary phase HPLC analysis using a ChiralPak IC column (80:20 hexanes/i-PrOH at $1.0 \mathrm{~mL} / \mathrm{min})$, major isomer: $\mathrm{t}_{\mathrm{R}}=17.492 \mathrm{~min}$, minor isomer: $\mathrm{t}_{\mathrm{R}}=10.183 \mathrm{~min}$; HRMS $(\mathrm{m} / \mathrm{z})$ : calcd for $\mathrm{C}_{35} \mathrm{H}_{34} \mathrm{FN}_{2} \mathrm{O}_{4} \mathrm{~S}\left(\left[\mathrm{M}+\mathrm{NH}_{4}\right]^{+}\right): 597.2218$; found: 597.2208 .

\section{2-Methylnaphthalen-1-yl}

$(S, E)-2-\{(S)$-(4-chlorophenyl)[(4-methylphenyl)sulfonamido]methyl\}-4-phenylbut -3-enoate (9h)<smiles>Cc1ccc2ccccc2c1OC(=O)[C@H](/C=C/c1ccccc1)[C@H](NC(F)(F)F)c1ccc(Cl)cc1</smiles>

White solid; $47.6 \mathrm{mg}, 80 \%$ yield; m.p. $220-222{ }^{\circ} \mathrm{C}$ $[\alpha]_{\mathrm{D}}^{25}=-115.0(\mathrm{c} 0.95, \mathrm{THF}, 84: 16 \mathrm{dr}, 83 \%$ ee $) ;{ }^{1} \mathrm{H}$ $\left(500 \mathrm{MHz},\left(\mathrm{CD}_{3}\right)_{2} \mathrm{SO}\right): \delta 1.89(\mathrm{~s}, 3 \mathrm{H}), 2.26(\mathrm{~s}, 3 \mathrm{H})$, $4.15(\mathrm{t}, \mathrm{J}=9.5 \mathrm{~Hz}, 1 \mathrm{H}), 4.78(\mathrm{t}, \mathrm{J}=10.0 \mathrm{~Hz}, 1 \mathrm{H})$, $6.25(\mathrm{dd}, \mathrm{J}=7.0,16.0 \mathrm{~Hz}, 1 \mathrm{H}), 6.88(\mathrm{~d}, \mathrm{~J}=6.0 \mathrm{~Hz}$, 1H), $7.05(\mathrm{~d}, \mathrm{~J}=8.0 \mathrm{~Hz}, 2 \mathrm{H}), 7.14(\mathrm{t}, J=7.5 \mathrm{~Hz}, 1 \mathrm{H}), 7.30-7.44(\mathrm{~m}, 11 \mathrm{H}), 7.69(\mathrm{~d}, \mathrm{~J}$ $=8.5 \mathrm{~Hz}, 1 \mathrm{H}), 7.84(\mathrm{~d}, \mathrm{~J}=8.5 \mathrm{~Hz}, 1 \mathrm{H}), 8.50(\mathrm{~d}, \mathrm{~J}=9.5 \mathrm{~Hz}, 1 \mathrm{H}) ;{ }^{13} \mathrm{C}(125 \mathrm{MHz}$, $\left.\left(\mathrm{CD}_{3}\right)_{2} \mathrm{SO}\right): \delta 14.9,20.6,55.2,58.2,119.6,123.9,125.2,125.5,125.8,125.9,126.0$, $126.1,127.4,127.8,128.0,128.4,128.8,129.4,132.3,135.3,135.8,138.2,141.9$, 143.0, 168.7; $v_{\max }: 1086,1153,1327,1490,1598,1731,3319 \mathrm{~cm}^{-1}$; Enantiomeric excess of 9h was determined by chiral stationary phase HPLC analysis using a ChiralPak IC column (80:20 hexanes $/ i-\mathrm{PrOH}$ at $1.0 \mathrm{~mL} / \mathrm{min})$, major isomer: $\mathrm{t}_{\mathrm{R}}=17.5$ min, minor isomer: $t_{R}=12.6 \mathrm{~min} ; \operatorname{HRMS}(\mathrm{m} / \mathrm{z})$ : calcd for $\mathrm{C}_{35} \mathrm{H}_{34} \mathrm{ClN}_{2} \mathrm{O}_{4} \mathrm{~S}$ $\left(\left[\mathrm{M}+\mathrm{NH}_{4}\right]^{+}\right): 613.1922$; found: 613.1918 . 


\section{2-Methylnaphthalen-1-yl}

$(S, E)-2-\{(S)$-(4-bromophenyl)[(4-methylphenyl)sulfonamido]methyl\}-4-phenylbut -3-enoate (9i)<smiles>Cc1ccc2ccccc2c1OC(=O)[C@H](/C=C/c1ccccc1)[C@H](NC(F)(F)F)c1ccc(Br)cc1</smiles>

White solid; $47.6 \mathrm{mg}, 81 \%$ yield; m.p. $268-270{ }^{\circ} \mathrm{C}$ $[\alpha]_{\mathrm{D}}^{25}=-102.0(\mathrm{c} 0.95$, THF, 85:15 dr, 82\% ee) White solid; 81\% yield; m.p. 268-270 ${ }^{\circ} \mathrm{C} ;{ }^{1} \mathrm{H}(500 \mathrm{MHz}$, $\left.\left(\mathrm{CD}_{3}\right)_{2} \mathrm{SO}\right): \delta 1.89(\mathrm{~s}, 3 \mathrm{H}), 2.27(\mathrm{~s}, 3 \mathrm{H}), 4.15(\mathrm{t}, J=$ $10.0 \mathrm{~Hz}, 1 \mathrm{H}), 4.76(\mathrm{t}, J=9.5 \mathrm{~Hz}, 1 \mathrm{H}), 6.25(\mathrm{dd}, J=$ 6.5, 15.5 Hz, 1H), $6.88(\mathrm{~d}, J=15.5 \mathrm{~Hz}, 1 \mathrm{H}), 7.05(\mathrm{~d}, J=8.0 \mathrm{~Hz}, 2 \mathrm{H}), 7.17$ (t, $J=7.5$ $\mathrm{Hz}, 1 \mathrm{H}), 7.25$ (d, $J=8.5 \mathrm{~Hz}, 2 \mathrm{H}), 7.30-7.45(\mathrm{~m}, 10 \mathrm{H}), 7.69$ (d, $J=8.5 \mathrm{~Hz}, 1 \mathrm{H}), 7.84$ $(\mathrm{d}, J=8.5 \mathrm{~Hz}, 1 \mathrm{H}), 8.49(\mathrm{~d}, J=9.0 \mathrm{~Hz}, 1 \mathrm{H}) ;{ }^{13} \mathrm{C}\left(125 \mathrm{MHz},\left(\mathrm{CD}_{3}\right)_{2} \mathrm{SO}\right): \delta 14.9,20.6$, 55.1, 58.2, 119.6, 120.8, 123.9, 125.2, 125.5, 125.9, 126.0, 126.1, 127.4, 127.8, 128.4, $128.8,129.8,130.9,132.3,135.3,135.8,138.1,138.5,141.9,142.9,168.6 ; v_{\max }: 1074$ $1147,1332,1445,1488,1597,1732,3319 \mathrm{~cm}^{-1}$; Enantiomeric excess of 9i was determined by chiral stationary phase HPLC analysis using a ChiralPak IC column (80:20 hexanes $/ i-\mathrm{PrOH}$ at $1.0 \mathrm{~mL} / \mathrm{min})$, major isomer: $\mathrm{t}_{\mathrm{R}}=18.7 \mathrm{~min}$, minor isomer: $\mathrm{t}_{\mathrm{R}}$ $=13.1$ min. HRMS $(m / z)$ : calcd for $\mathrm{C}_{35} \mathrm{H}_{30} \mathrm{BrNNaO}_{4} \mathrm{~S}\left([\mathrm{M}+\mathrm{Na}]^{+}\right)$: 662.0971; found: 662.0990 .

\section{2-Methylnaphthalen-1-yl}

$(S, E)-2-\{(S)$-[(4-methylphenyl)sulfonamido][4-(trifluoromethyl)phenyl]methyl $\}-4$ -phenylbut-3-enoate (9j)<smiles>Cc1ccc2ccccc2c1OC(=O)C(/C=C/c1ccccc1)C(N[As])c1ccc(C(F)(F)F)cc1</smiles>
White solid; $52.04 \mathrm{mg}, 83 \%$ yield; m.p. $234-236{ }^{\circ} \mathrm{C}$ $[\alpha]_{\mathrm{D}}{ }^{25}=-168.6(\mathrm{c} 1.0, \mathrm{THF}, 96: 4 \mathrm{dr}, 97 \%$ ee $) ;{ }^{1} \mathrm{H}$ $\left(500 \mathrm{MHz},\left(\mathrm{CD}_{3}\right)_{2} \mathrm{SO}\right): \delta 1.83(\mathrm{~s}, 3 \mathrm{H}), 2.21(\mathrm{~s}, 3 \mathrm{H})$, $4.21(\mathrm{t}, J=10.0 \mathrm{~Hz}, 1 \mathrm{H}), 4.88(\mathrm{t}, J=9.5 \mathrm{~Hz}, 1 \mathrm{H})$, $6.31(\mathrm{dd}, J=6.5,15.5 \mathrm{~Hz}, 1 \mathrm{H}), 6.90(\mathrm{~d}, J=16.0 \mathrm{~Hz}$ 1H), 7.00-7.07 (m, 3H), 7.29-7.57 (m, 12H), 7.69 (d, $J=8.0 \mathrm{~Hz}, 1 \mathrm{H}), 7.84$ (d, $J=8.0$ $\mathrm{Hz}, 1 \mathrm{H}), 8.59(\mathrm{~d}, J=9.0 \mathrm{~Hz}, 1 \mathrm{H}) ;{ }^{13} \mathrm{C}\left(125 \mathrm{MHz},\left(\mathrm{CD}_{3}\right)_{2} \mathrm{SO}\right): \delta 14.8,20.4,54.9,58.4$, $119.5,123.7,124.8,125.2,125.6,125.8,125.9,126.0,126.2,127.4,127.9,128.4$, $128.5,128.7,132.3,135.5,135.8,138.0,141.8,142.9,143.5,168.5$; $v_{\max }: 1017,1087$, $1116,1155,1326,1451,1599,1729,3319 \mathrm{~cm}^{-1}$; Enantiomeric excess of $\mathbf{9 j}$ was 
determined by chiral stationary phase HPLC analysis using a ChiralPak IC column (80:20 hexanes $/ i-\mathrm{PrOH}$ at $1.0 \mathrm{~mL} / \mathrm{min})$, major isomer: $\mathrm{t}_{\mathrm{R}}=12.9 \mathrm{~min}$, minor isomer: $\mathrm{t}_{\mathrm{R}}$ $=8.2$ min. HRMS $(\mathrm{m} / z)$ calcd for $\mathrm{C}_{36} \mathrm{H}_{34} \mathrm{~F}_{3} \mathrm{~N}_{2} \mathrm{O}_{4} \mathrm{~S}\left(\left[\mathrm{M}+\mathrm{NH}_{4}\right]^{+}\right)$: 647.2186; found: 647.2191 .

\section{2-Methylnaphthalen-1-yl}

$(S, E)-2-\{(S)$-(2-chlorophenyl)[(4-methylphenyl)sulfonamide]methyl\}-4-phenylbut -3-enoate (9k)<smiles>Cc1ccc2ccccc2c1OC(=O)[C@H](/C=C/c1ccccc1)[C@H](NS)c1ccccc1Cl</smiles>

White solid; $38.5 \mathrm{mg}, 65 \%$ yield; m.p. $162-164{ }^{\circ} \mathrm{C}[\alpha]_{\mathrm{D}}{ }^{25}$ $=-148.2$ (c 1.0, THF, 80:20 dr, 78\% ee); ${ }^{1} \mathrm{H}(500 \mathrm{MHz}$, $\mathrm{CDCl}_{3}: \delta 1.90(\mathrm{~s}, 3 \mathrm{H}), 2.30(\mathrm{~s}, 3 \mathrm{H}), 4.39(\mathrm{~s}, 1 \mathrm{H}), 5.24(\mathrm{~s}$, $1 \mathrm{H}), 5.63(\mathrm{~d}, J=8.0 \mathrm{~Hz}, 1 \mathrm{H}), 6.18(\mathrm{dd}, J=6.5,15.5 \mathrm{~Hz}$, 1H), $6.82(\mathrm{~d}, J=16.0 \mathrm{~Hz}, 1 \mathrm{H}), 7.00(\mathrm{~d}, J=8.0 \mathrm{~Hz}, 2 \mathrm{H})$, 7.13-7.38 (m, 13H), $7.57(\mathrm{t}, J=8.5 \mathrm{~Hz}, 1 \mathrm{H}), 7.72(\mathrm{~d}, J=$ $8.0 \mathrm{~Hz}, 1 \mathrm{H}) ;{ }^{13} \mathrm{C}\left(125 \mathrm{MHz}, \mathrm{CDCl}_{3}\right): \delta 15.8,21.4,54.1,120.3,125.4,126.0,126.4$, $126.5,126.7,126.8,127.1,127.2,127.7,128.4,128.5,128.7,129.3,129.4,130.4$, 133.1, 136.1, 137.0, 143.2, 143.7; v $v_{\max }: 1077,1159,1325,1439,1597,1749,2920$, $3239 \mathrm{~cm}^{-1}$; Enantiomeric excess of 9k was determined by chiral stationary phase HPLC analysis using a ChiralPak IC column (80:20 hexanes/ $i-\mathrm{PrOH}$ at $1.0 \mathrm{~mL} / \mathrm{min}$ ), major isomer: $t_{R}=84.2 \mathrm{~min}$, minor isomer: $t_{R}=21.7 \mathrm{~min}$. HRMS $(\mathrm{m} / \mathrm{z})$ calcd for $\mathrm{C}_{35} \mathrm{H}_{34} \mathrm{ClN}_{2} \mathrm{O}_{4} \mathrm{~S}\left(\left[\mathrm{M}+\mathrm{NH}_{4}\right]^{+}\right): 613.1922$; found: 613.1929 .

\section{2-Methylnaphthalen-1-yl}

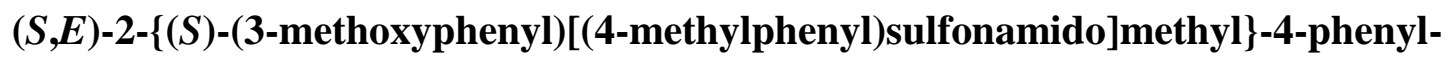
but-3-enoate (9I)<smiles>COc1cccc([C@H](NC(=O)c2ccccc2)[C@H](/C=C/c2ccccc2)C(=O)Oc2c(C)ccc3ccccc23)c1</smiles>

White solid; $41.9 \mathrm{mg}, 71 \%$ yield; m.p. $237-238^{\circ} \mathrm{C}[\alpha]_{\mathrm{D}}{ }^{25}$ $=-129.0(\mathrm{c} 1.0$, THF, 84:16 dr, 86\% ee $) ;{ }^{1} \mathrm{H}(500 \mathrm{MHz}$, $\left(\mathrm{CDCl}_{3}\right): \delta 1.87(\mathrm{~s}, 3 \mathrm{H}), 2.33(\mathrm{~s}, 3 \mathrm{H}), 3.68(\mathrm{~s}, 3 \mathrm{H}), 4.02$ (t, $J=10.0 \mathrm{~Hz}, 1 \mathrm{H}), 4.82(\mathrm{dd}, J=8.0,10.0 \mathrm{~Hz}, 1 \mathrm{H}), 6.20$ $(\mathrm{dd}, J=6.5,15.5 \mathrm{~Hz}, 1 \mathrm{H}), 6.73(\mathrm{~s}, 1 \mathrm{H}), 6.89(\mathrm{t}, J=15.0$ $\mathrm{Hz}, 3 \mathrm{H}), 7.04$ (d, $J=8.0 \mathrm{~Hz}, 3 \mathrm{H}), 7.19$ (d, $J=8.0 \mathrm{~Hz}, 2 \mathrm{H}), 7.26$ (s, 1H), 7.30-7.39 (m, $7 \mathrm{H}), 7.52(\mathrm{~d}, J=8.0 \mathrm{~Hz} 1 \mathrm{H}), 7.57(\mathrm{~d}, J=8.0 \mathrm{~Hz} 1 \mathrm{H}), 7.71(\mathrm{~d}, J=8.0 \mathrm{~Hz} 1 \mathrm{H}) ;{ }^{13} \mathrm{C}$ 
(125 MHz, $\left.\left(\mathrm{CD}_{3}\right)_{2} \mathrm{SO}\right): \delta 15.7,21.3,55.4,56.1,59.4,113.6,113.8,120.5,125.1$, $125.9,126.2$, 126.6, 126.7, 126.9, 128.5, 129.1, 129.2,129.4, 132.9, 135.8, 136.6, $138.9,141.2,142.4,143.7,159.6,169.5$; v $v_{\max }: 1027,1072,1144,1253,1328,1445$, 1511, 1613, 1728, $3312 \mathrm{~cm}^{-1}$; Enantiomeric excess of 91 was determined by chiral stationary phase HPLC analysis using a ChiralPak IC column (80:20 hexanes/i-PrOH at $1.0 \mathrm{~mL} / \mathrm{min}$ ), major isomer: $\mathrm{t}_{\mathrm{R}}=30.2 \mathrm{~min}$, minor isomer: $\mathrm{t}_{\mathrm{R}}=23.7 \mathrm{~min}$; HRMS $(\mathrm{m} / z)$ calcd for $\mathrm{C}_{36} \mathrm{H}_{33} \mathrm{NNaO}_{5} \mathrm{~S}\left([\mathrm{M}+\mathrm{Na}]^{+}\right)$: 614.1972; found: 614.1985 .

\section{2-Methylnaphthalen-1-yl}

$(2 S, 3 R, E)-3-[(4-m e t h y l p h e n y l)$ sulfonamido]-5-phenyl-2-[(E)-styryl)pent-4-enoate $(9 \mathrm{~m})$<smiles>Cc1ccc2ccccc2c1OC(=O)C(/C=C/c1ccccc1)[C@H](/C=C/c1ccccc1)NC(F)(F)F</smiles>

White solid; $46.3 \mathrm{mg}, 72 \%$ yield m.p. $180-182{ }^{\circ} \mathrm{C}$ $[\alpha]_{\mathrm{D}}^{25}=-131.0(\mathrm{c} 0.5, \mathrm{THF}, 80: 20 \mathrm{dr}, 76 \%$ ee $) ;{ }^{1} \mathrm{H}$ $\left(500 \mathrm{MHz},\left(\mathrm{CD}_{3}\right)_{2} \mathrm{SO}\right): \delta 2.10(\mathrm{~s}, 3 \mathrm{H}), 2.14(\mathrm{~s}, 3 \mathrm{H})$, $3.97(\mathrm{t}, J=8.5 \mathrm{~Hz}, 1 \mathrm{H}), 4.42(\mathrm{q}, J=10.0 \mathrm{~Hz}, 1 \mathrm{H})$, $6.01(\mathrm{q}, J=8.5 \mathrm{~Hz}, 1 \mathrm{H}), 6.27(\mathrm{~d}, J=15 \mathrm{~Hz}, 1 \mathrm{H})$, $6.33(\mathrm{q}, J=10.5 \mathrm{~Hz}, 1 \mathrm{H}), 6.84(\mathrm{~d}, J=10.5 \mathrm{~Hz}, 1 \mathrm{H}), 7.14-7.16(\mathrm{~m}, 4 \mathrm{H}), 7.26-7.40(\mathrm{~m}$, 10H), 7.47 (d, $J=8.0 \mathrm{~Hz}, 2 \mathrm{H}), 7.63(\mathrm{~d}, J=10.0 \mathrm{~Hz}, 1 \mathrm{H}), 7.72(\mathrm{~d}, J=8.5 \mathrm{~Hz}, 1 \mathrm{H})$, $7.83(\mathrm{~d}, J=8.5 \mathrm{~Hz}, 1 \mathrm{H}), 8.14(\mathrm{~d}, J=8.5 \mathrm{~Hz}, 1 \mathrm{H}) ;{ }^{13} \mathrm{C}\left(125 \mathrm{MHz},\left(\mathrm{CD}_{3}\right)_{2} \mathrm{SO}\right): \delta 14.9$, 20.6, 55.4, 58.8, 119.8, 124.4, 125.2, 125.4, 125.9, 126.1, 127.2, 127.4, 127.5, 127.7, $128.0,128.3,128.4,128.7,132.2,135.1,135.9,138.4,139.4,141.7,143.0,168.7$; $v_{\max }: 1085,1149,1334,1447,1494,1600,1732,3321 \mathrm{~cm}^{-1}$; Enantiomeric excess of 9m was determined by chiral stationary phase HPLC analysis using a ChiralPak IC column $(80: 20$ hexanes $/ i-\mathrm{PrOH}$ at $1.0 \mathrm{~mL} / \mathrm{min})$, major isomer: $\mathrm{t}_{\mathrm{R}}=19.1 \mathrm{~min}$, minor isomer: $\mathrm{t}_{\mathrm{R}}=23.7 \mathrm{~min}$; HRMS $(\mathrm{m} / z)$ : calcd for $\mathrm{C}_{37} \mathrm{H}_{37} \mathrm{~N}_{2} \mathrm{O}_{4} \mathrm{~S}\left(\left[\mathrm{M}+\mathrm{NH}_{4}\right]^{+}\right): 605.2469$; found: 605.2469 .

\section{2-Methylnaphthalen-1-yl}

$(S, E)-2-\{(S)$-[(4-methylphenyl)sulfonamido](phenyl)methyl $\}-4-(p$-tolyl)but-3-enoate (9n)

White solid; $42.0 \mathrm{mg}, 73 \%$ yield m.p. $239-241{ }^{\circ} \mathrm{C}[\alpha]_{\mathrm{D}}{ }^{25}=-262.8(\mathrm{c} 1.02$, THF, 83:17 dr, 73\% ee) ${ }^{1} \mathrm{H}\left(500 \mathrm{MHz},\left(\mathrm{CD}_{3}\right)_{2} \mathrm{SO}\right): \delta 1.97$ (s, 3H), 2.24 (s, 3H), 2.26 (s, 3H), 4.13 
$(\mathrm{t}, J=10.0 \mathrm{~Hz}, 1 \mathrm{H}), 4.72 \quad(\mathrm{~d}, J=10.5 \mathrm{~Hz}, 1 \mathrm{H}), 6.11(\mathrm{t}, J=15.5 \mathrm{~Hz}, 1 \mathrm{H}), 6.83(\mathrm{~d}, J=$<smiles>Cc1ccc(/C=C/[C@H](C(=O)Oc2c(C)ccc3ccccc23)[C@H](N[As])c2ccccc2)cc1</smiles>
$15.0 \mathrm{~Hz}, 1 \mathrm{H}), 7.02(\mathrm{~d}, J=8.0 \mathrm{~Hz}, 2 \mathrm{H}), 7.20(\mathrm{~d}, \mathrm{~J}=8.0$ $\mathrm{Hz}, 3 \mathrm{H}), 7.24-7.28(\mathrm{~m}, 7 \mathrm{H}), 7.30(\mathrm{t}, J=8.0 \mathrm{~Hz}, 2 \mathrm{H})$, $7.38(\mathrm{~d}, J=10.0 \mathrm{~Hz}, 2 \mathrm{H}), 7.41(\mathrm{~d}, J=8.0 \mathrm{~Hz}, 1 \mathrm{H}), 7.66$ $(\mathrm{d}, J=8.5 \mathrm{~Hz}, 1 \mathrm{H}), 8.56(\mathrm{~d}, J=10.0 \mathrm{~Hz}, 1 \mathrm{H}) ;{ }^{13} \mathrm{C}(125$ $\left.\mathrm{MHz},\left(\mathrm{CD}_{3}\right)_{2} \mathrm{SO}\right): \delta 15.6,21.3,56.2,59.5,120.4,123.9$, $125.9,126.2,126.6,126.8,126.9,128.0,128.1,128.2$, $128.8,129.0,129.5,129.7,132.9,133.8,135.7,137.9,139.0,140.1,142.4,143.7$, 169.6; $v_{\max }: 1086,1146,1330,1454,1511,1600,1731,3320 \mathrm{~cm}^{-1}$; Enantiomeric excess of 9n was determined by chiral stationary phase HPLC analysis using a ChiralPak IC column (80:20 hexanes $/ i-\mathrm{PrOH}$ at $1.0 \mathrm{~mL} / \mathrm{min})$, major isomer: $\mathrm{t}_{\mathrm{R}}=30.3$ min, minor isomer: $t_{R}=19.8$ min; HRMS $(m / z)$ calcd for $\mathrm{C}_{36} \mathrm{H}_{37} \mathrm{~N}_{2} \mathrm{O}_{4} \mathrm{~S}\left(\left[\mathrm{M}+\mathrm{NH}_{4}\right]^{+}\right)$: 593.2469; found: 593.2459 .

\section{2-Methylnaphthalen-1-yl}

$(S, E)-4-(4-m e t h o x y p h e n y l)-2-\{(S)-[(4-m e t h y l p h e n y l) s u l f o n a m i d o](p h e n y l)-$ methyl \}but-3-enoate (9o)<smiles>[CH]N[C@H](c1ccccc1)[C@H](/C=C/c1ccc(OC)cc1)C(=O)Oc1c(C)ccc2ccccc12</smiles>

White solid; $49.6 \mathrm{mg}, 84 \%$ yield m.p. $218-220^{\circ} \mathrm{C}[\alpha]_{\mathrm{D}}{ }^{25}$ $=-255.8$ (c 1.0, THF, 89:11 dr, 86\% ee); ${ }^{1} \mathrm{H}$ NMR $(500$ $\left.\mathrm{MHz},\left(\mathrm{CD}_{3}\right)_{2} \mathrm{SO}\right): \delta 1.90(\mathrm{~s}, 3 \mathrm{H}), 2.24(\mathrm{~s}, 3 \mathrm{H}), 3.80(\mathrm{~s}$, $3 \mathrm{H}), 4.10(\mathrm{t}, J=9.9 \mathrm{~Hz}, 1 \mathrm{H}), 4.72(\mathrm{t}, J=9.4 \mathrm{~Hz}, 1 \mathrm{H})$, $6.03(\mathrm{dd}, J=15.3,9.3 \mathrm{~Hz}, 1 \mathrm{H}), 6.82(\mathrm{t}, J=10.7 \mathrm{~Hz}, 1 \mathrm{H})$, $6.96(\mathrm{~d}, J=8.6 \mathrm{~Hz}, 2 \mathrm{H}), 7.03(\mathrm{~d}, J=8.1 \mathrm{~Hz}, 2 \mathrm{H}), 7.38-$ $7.21(\mathrm{~m}, 10 \mathrm{H}), 7.42(\mathrm{~d}, J=8.1 \mathrm{~Hz}, 2 \mathrm{H}), 7.67(\mathrm{~d}, J=8.4 \mathrm{~Hz}, 1 \mathrm{H}), 7.81(\mathrm{~d}, J=8.2 \mathrm{~Hz}$, $1 \mathrm{H}), 8.54(\mathrm{~d}, J=9.5 \mathrm{~Hz}, 1 \mathrm{H}) ;{ }^{13} \mathrm{C} \mathrm{NMR}\left(125 \mathrm{MHz},\left(\mathrm{CD}_{3}\right)_{2} \mathrm{SO}\right): \delta 20.9,55.2,55.8$, 59.0, 114.1, $120.0122 .1,125.5,125.7,126.2,126.4,127.6,127.7,127.8,128.3,128.6$, $128.7,129.0,132.4,134.9,138.6,139.7,141.9,143.2,159.2,169.2 . v_{\max }: 1085,1149$, 1334, 1447, 1494, 1600, 1732, $3321 \mathrm{~cm}^{-1}$; Enantiomeric excess of 90 was determined by chiral stationary phase HPLC analysis using a ChiralPak IC column (80:20 hexanes $/ i-\mathrm{PrOH}$ at $1.0 \mathrm{~mL} / \mathrm{min}$ ), major isomer: $\mathrm{t}_{\mathrm{R}}=24.2 \mathrm{~min}$, minor isomer: $\mathrm{t}_{\mathrm{R}}=17.1$ min; HRMS $(m / z)$ calcd for $\mathrm{C}_{36} \mathrm{H}_{37} \mathrm{~N}_{2} \mathrm{O}_{5} \mathrm{~S}\left(\left[\mathrm{M}+\mathrm{NH}_{4}\right]^{+}\right)$: 609.2418; found: 609.2426. 


\section{2-Methylnaphthalen-1-yl}

\section{$(S, E)-4-(4-f l u o r o p h e n y l)-2-\{(S)-[(4-m e t h y l p h e n y l) s u l f o n a m i d o](p h e n y l) m e t h y l\}$}

but-3-enoate (9p)<smiles>Cc1ccc2ccccc2c1OC(=O)C(/C=C/c1ccc(F)cc1)[C@H](NS)c1ccccc1</smiles>
White solid; $46.3 \mathrm{mg}, 80 \%$ yield m.p. $259-261^{\circ} \mathrm{C}[\alpha]_{\mathrm{D}}{ }^{25}$ $=-298.2\left(\mathrm{c} 1.0\right.$, THF, 95:5 dr, 92\% ee) ${ }^{1} \mathrm{H}(500 \mathrm{MHz}$, $\left.\left(\mathrm{CD}_{3}\right)_{2} \mathrm{SO}\right): \delta 1.85(\mathrm{~s}, 3 \mathrm{H}), 2.23(\mathrm{~s}, 3 \mathrm{H}), 4.19(\mathrm{t}, J=10.0$ $\mathrm{Hz}, 1 \mathrm{H}), 4.80(\mathrm{t}, J=10.5 \mathrm{~Hz}, 1 \mathrm{H}), 6.24(\mathrm{dd}, J=6.5,15.5$ $\mathrm{Hz}, 1 \mathrm{H}), 6.89(\mathrm{~d}, J=16.0 \mathrm{~Hz}, 1 \mathrm{H}), 7.04(\mathrm{~d}, J=8.0 \mathrm{~Hz}$, 2H), $7.10(\mathrm{t}, J=7.5 \mathrm{~Hz}, 1 \mathrm{H}), 7.26-7.46(\mathrm{~m}, 13 \mathrm{H}), 7.67(\mathrm{~d}$, $J=8.5 \mathrm{~Hz}, 1 \mathrm{H}), 7.82(\mathrm{~d}, J=8.0 \mathrm{~Hz}, 1 \mathrm{H}) ;{ }^{13} \mathrm{C}\left(125 \mathrm{MHz},\left(\mathrm{CD}_{3}\right)_{2} \mathrm{SO}\right): \delta 20.9,55.4$, $58.8,124.3,125.6,125.8,126.3,126.4,128.1,128.7,128.8,129.1,132.5,135.5$, 136.1, 128.4, 142.1, 143.1, 169.1; $v_{\max }$ : 1085, 1149, 1334, 1447, 1494, 1600, 1732, $3321 \mathrm{~cm}^{-1}$; Enantiomeric excess of 9p was determined by chiral stationary phase HPLC analysis using a ChiralPak IC column (80:20 hexanes/i-PrOH at $1.0 \mathrm{~mL} / \mathrm{min})$, major isomer: $t_{R}=19.2 \mathrm{~min}$, minor isomer: $t_{R}=14.2 \mathrm{~min} ; \operatorname{HRMS}(\mathrm{m} / \mathrm{z})$ : calcd for $\mathrm{C}_{35} \mathrm{H}_{34} \mathrm{FN}_{2} \mathrm{O}_{4} \mathrm{~S}\left(\left[\mathrm{M}+\mathrm{NH}_{4}\right]^{+}\right): 597.2218$; found: 597.2234.

\section{2-Methylnaphthalen-1-yl}

$(S, E)-4-(4-c h l o r o p h e n y l)-2-\{[(S)-(4-m e t h y l p h e n y l s u l f o n a m i d o)](p h e n y l) m e t h y l ~\}$ but-3-enoate (9q)<smiles>Cc1ccc2ccccc2c1OC(=O)C(/C=C/c1ccc(Cl)cc1)[C@H](N[Te])c1ccccc1</smiles>

White solid, $49.8 \mathrm{mg}, 84 \%$ yield; $\mathrm{mp}: 231-232{ }^{\circ} \mathrm{C} ;[\alpha]_{\mathrm{D}}^{25}$ $=-221$ (c 1.0, THF, 92:8 dr, 90\% ee); ${ }^{1} \mathrm{H}$ NMR $(500$ $\left.\mathrm{MHz},\left(\mathrm{CD}_{3}\right)_{2} \mathrm{SO}\right): \delta 2.19(\mathrm{~s}, 3 \mathrm{H}), 2.35(\mathrm{~s}, 3 \mathrm{H}), 4.14(\mathrm{t}, J=$ $10.0 \mathrm{~Hz}, 1 \mathrm{H}), 4.76(\mathrm{t}, J=8.5 \mathrm{~Hz}, 1 \mathrm{H}), 6.21-6.25(\mathrm{~m}, 1 \mathrm{H})$, $6.85(\mathrm{~d}, J=15.5 \mathrm{~Hz}, 1 \mathrm{H}), 7.01(\mathrm{~d}, J=8.5 \mathrm{~Hz}, 1 \mathrm{H}), 7.25-$ $7.43(\mathrm{~m}, 12 \mathrm{H}), 7.65-7.70(\mathrm{~m}, 3 \mathrm{H}), 7.80(\mathrm{~d}, J=8.5 \mathrm{~Hz}$, $1 \mathrm{H}), 8.58(\mathrm{~d}, J=9.5 \mathrm{~Hz}, 1 \mathrm{H}) ;{ }^{13} \mathrm{C} \mathrm{NMR}\left(125 \mathrm{MHz},\left(\mathrm{CD}_{3}\right)_{2} \mathrm{SO}\right) \delta 21.3,21.4,56.2,59.5$, $120.4,126.0,126.5,128.2,128.6,129.1,129.5,129.7,132.9,133.0,134.6,135.4$, 139.0, 139.9, 141.8, 142.3, 142.4, 143.6, 169.4; v $v_{\max }: 1013,1066,1089,1156,1184$, $1288,1325,1491,1596,1687,1736,3279 \mathrm{~cm}^{-1}$; Enantiomeric excess of 9q was determined by chiral stationary phase HPLC analysis using a ChiralPak ID column 
(80:20 hexanes $/ i-\mathrm{PrOH}$ at $1.0 \mathrm{~mL} / \mathrm{min}, \lambda=220 \mathrm{~nm}$ ), major enantiomer: $\mathrm{t}_{\mathrm{R}}=44.0 \mathrm{~min}$; minor enantiomer: $t_{R}=34.6$ min.; HRMS $(\mathrm{m} / \mathrm{z})$ calcd for $\mathrm{C}_{35} \mathrm{H}_{30} \mathrm{ClNNaO}_{3} \mathrm{~S}$ $\left([\mathrm{M}+\mathrm{Na}]^{+}\right): 618.1476$; found: 618.1484 .

\section{2-Methylnaphthalen-1-yl}

$(S, E)-4-(4-b r o m o p h e n y l)-2-\{[(S)-((4-m e t h y l p h e n y l)$ sulfonamido $)]($ phenyl)methyl $\}$ but-3-enoate (9r)<smiles>Cc1ccc2ccccc2c1OC(=O)[C@H](/C=C/c1ccc(Br)cc1)[C@H](N[Te])c1ccccc1</smiles>

White solid, $53.1 \mathrm{mg}, 83 \%$ yield; m.p. $229-230^{\circ} \mathrm{C} ;[\alpha]_{\mathrm{D}}{ }^{21}=$ -213.7 (c 1.0, $\mathrm{CHCl}_{3}, 87: 13 \mathrm{dr}, 96 \%$ ee); ${ }^{1} \mathrm{H}$ NMR (500 $\left.\mathrm{MHz},\left(\mathrm{CD}_{3}\right)_{2} \mathrm{SO}\right) \delta 2.20(\mathrm{~s}, 3 \mathrm{H}), 2.49(\mathrm{~s}, 3 \mathrm{H}), 4.15(\mathrm{t}, J=$ $10.0 \mathrm{~Hz}, 1 \mathrm{H}), 4.77$ (t, $J=10 \mathrm{~Hz}, 1 \mathrm{H}), 6.23-6.28(\mathrm{~m}, 1 \mathrm{H})$, $6.84(\mathrm{~d}, J=16.0 \mathrm{~Hz}, 1 \mathrm{H}), 7.02(\mathrm{~d}, J=8.0 \mathrm{~Hz}, 1 \mathrm{H}), 7.27-$ $7.44(\mathrm{~m}, 12 \mathrm{H}), 7.58-7.59(\mathrm{~m}, 1 \mathrm{H}), 7.67-7.72(\mathrm{~m}, 2 \mathrm{H}), 7.82$ $(\mathrm{d}, J=7.0 \mathrm{~Hz}, 1 \mathrm{H}), 8.59(\mathrm{~d}, J=9.5 \mathrm{~Hz}, 1 \mathrm{H}) ;{ }^{13} \mathrm{C} \mathrm{NMR}\left(125 \mathrm{MHz},\left(\mathrm{CD}_{3}\right)_{2} \mathrm{SO}\right) \delta 15.6$, 21.3, 56.2, 59.5, 120.4, 121.6, 125.9, 126.0, 126.1, 126.2, 126.5, 126.6, 126.9, 128.0, 128.2, 128.8, 128.9, 129.1, 129.5, 129.7, 132.1, 132.9, 134.7, 135.8, 139.0, 139.9, $141.9,142.3,142.4,143.6,169.4 ; v_{\max }: 1011,1089,1159,1236,1263,1489,1732$, $3318 \mathrm{~cm}^{-1}$; Enantiomeric excess of 9r was determined by chiral stationary phase HPLC analysis using a ChiralPak IC column (80:20 hexanes $/ i-\mathrm{PrOH}$ at $0.8 \mathrm{~mL} / \mathrm{min}, \lambda=220$ $\mathrm{nm}$ ), major enantiomer: $\mathrm{t}_{\mathrm{R}}=26.8 \mathrm{~min}$; minor enantiomer: $\mathrm{t}_{\mathrm{R}}=18.5 \mathrm{~min}$. HRMS (ESI): $m / z$ calcd for $\mathrm{C}_{35} \mathrm{H}_{30} \mathrm{BrNNaO}_{4} \mathrm{~S}\left([\mathrm{M}+\mathrm{Na}]^{+}\right) 662.0971$, found 662.0963 .

\section{2-Methylnaphthalen-1-yl}

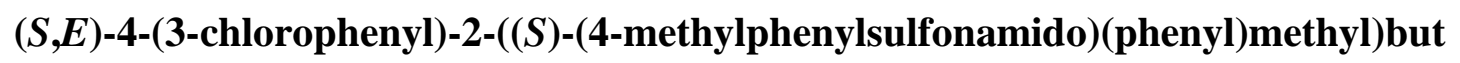
-3-enoate (9s)<smiles>Cc1ccc2ccccc2c1OC(=O)C(/C=C/c1cccc(Cl)c1)[C@H](NS)c1ccccc1</smiles>

White solid, $48.6 \mathrm{mg}, 82 \%$ yield; mp: $227-229{ }^{\circ} \mathrm{C} ;[\alpha]_{\mathrm{D}}{ }^{25}$ $=-262$ (c 1.0, THF, 94: 6 dr, 92\% ee); ${ }^{1} \mathrm{H}$ NMR $(300$ $\left.\mathrm{MHz},\left(\mathrm{CD}_{3}\right)_{2} \mathrm{CO}\right) \delta 1.91(\mathrm{~s}, 3 \mathrm{H}), 2.29(\mathrm{~s}, 3 \mathrm{H}), 4.23-4.36$ $(\mathrm{m}, 1 \mathrm{H}), 4.95(\mathrm{t}, J=10.0 \mathrm{~Hz}, 1 \mathrm{H}), 6.35(\mathrm{dd}, J=9.4,15.8$ $\mathrm{Hz}, 1 \mathrm{H}), 6.93(\mathrm{~d}, J=15.8 \mathrm{~Hz}, 1 \mathrm{H}), 7.05-7.11(\mathrm{~m}$, 3H), $7.22-7.59(\mathrm{~m}, 19 \mathrm{H}), 7.66(\mathrm{~d}, J=8.4 \mathrm{~Hz}, 1 \mathrm{H}), 7.80(\mathrm{~d}, J=8.2 \mathrm{~Hz}, 1 \mathrm{H}) ;{ }^{13} \mathrm{C}$ NMR $\left(75 \mathrm{MHz},\left(\mathrm{CD}_{3}\right)_{2} \mathrm{CO}\right) \delta 15,20.4,56.4,59.5,120.5,125.2,125.4,125.8,126.3$, 
$126.4,126.6,126.7,127.5,127.9,128.1,128.5,129.2,130.3,133,134.1,134.5$, 138.7, 138.9, 139.9 142.5, 143.8, 168.8; v $v_{\max }$ : 1013, 1064, 1091, 1152, 1182, 1288, 1325, 1492, 1594, 1685, 1737, $3271 \mathrm{~cm}^{-1}$; Enantiomeric excess of 9s was determined by chiral stationary phase HPLC analysis using a ChiralPak IC column (80: 20 hexane $/ i-\mathrm{PrOH}$ at $1.0 \mathrm{~mL} / \mathrm{min}, \lambda=254 \mathrm{~nm}$ ), major enantiomer: $t_{\mathrm{R}}=22.7 \mathrm{~min}$, minor enantiomer: $t_{R}=15.7 \mathrm{~min} ; \operatorname{HRMS}(\mathrm{m} / \mathrm{z})$ calcd for $\mathrm{C}_{35} \mathrm{H}_{30} \mathrm{ClNNaO}_{4} \mathrm{~S}\left([\mathrm{M}+\mathrm{Na}]^{+}\right)$: 618.1476; found: 618.1491.

\section{2-Methylnaphthalen-1-yl}

$(S, E)-4-p h e n y l-2-[(S)$-phenyl( $N$-tosylbenzamido)methyl]but-3-enoate (10)

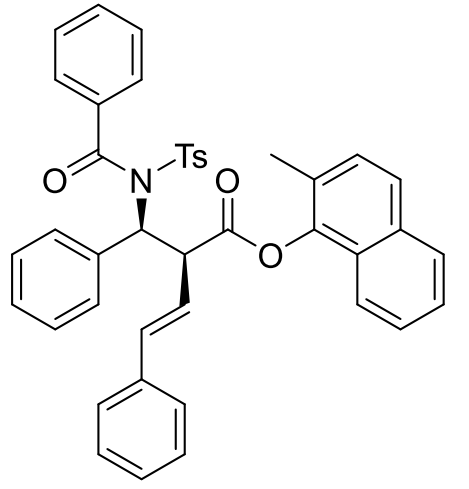

Colorless oil, $121.0 \mathrm{mg}, 91 \%$ yield; ${ }^{1} \mathrm{H}$ NMR (500 MHz, $\left.\mathrm{CDCl}_{3}\right) \delta 1.99(\mathrm{~s}, 3 \mathrm{H}), 2.32(\mathrm{~s}, 3 \mathrm{H}), 5.38(\mathrm{t}, J=10.5 \mathrm{~Hz}$, $1 \mathrm{H}), 6.32-6.33(\mathrm{~m}, 1 \mathrm{H}), 6.56(\mathrm{dd}, J=15.0,10 \mathrm{~Hz}, 1 \mathrm{H})$, 6.90-6.92 (m, 2H), 7.05-7.15 (m, 8H), $7.23(\mathrm{~d}, J=8.0 \mathrm{~Hz}$, 1H), 7.33-7.48 (m, 10H), $7.60(\mathrm{~d}, J=8.5 \mathrm{~Hz}, 1 \mathrm{H}), 7.75$ (d, $J=8.0 \mathrm{~Hz}, 1 \mathrm{H}), 7.97-7.99(\mathrm{~m}, 2 \mathrm{H}) ;{ }^{13} \mathrm{C} \mathrm{NMR}(125 \mathrm{MHz}$, $\left.\mathrm{CDCl}_{3}\right) \delta 15.9,21.5,53.1,120.5,124.4,125.3,125.9$, $126.5,126.8,127.4,127.6,128.2,128.3,128.5,128.6,128.7,128.8,129.2,130.6$, $130.7,133.0,136.0,136.1,136.2,136.9,143.8,144.5,169.8,172.1 ; v \max : 1085$, 1121, 1173, 1241, 1287, 1443, 1730,1756, $3354 \mathrm{~cm}^{-1}$; HRMS $(\mathrm{m} / \mathrm{z})$ calcd for $\mathrm{C}_{42} \mathrm{H}_{35} \mathrm{NNaO}_{5} \mathrm{~S}\left([\mathrm{M}+\mathrm{Na}]^{+}\right) 688.2128$, found 688.2136.

\section{2-Methylnaphthalen-1-yl}

(S,E)-2-[(S)-benzamido(phenyl)methyl]-4-phenylbut-3-enoate (11)

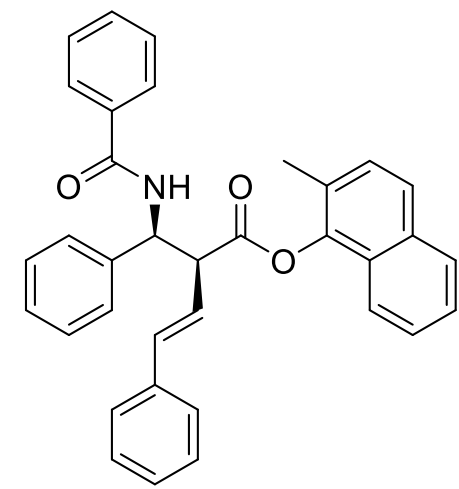

White solid, $43.4 \mathrm{mg}, 87 \%$ yield; $[\alpha]_{\mathrm{D}}^{23}=-83.9$ (c 1.0, $\left.\mathrm{CHCl}_{3}\right) ;{ }^{1} \mathrm{H} \mathrm{NMR}\left(500 \mathrm{MHz}, \mathrm{CDCl}_{3}\right) \delta 2.01$ (s, 3H), 4.35 $(\mathrm{t}, J=9.0 \mathrm{~Hz}, 1 \mathrm{H}), 5.93(\mathrm{t}, J=9 \mathrm{~Hz}, 1 \mathrm{H}), 6.57(\mathrm{dd}, J=$ 16.0, 9.5 Hz, 1H), $6.94(\mathrm{~d}, J=16.0 \mathrm{~Hz}, 1 \mathrm{H}), 7.09-7.19$ (m, $3 \mathrm{H}), 7.24-7.38(\mathrm{~m}, 6 \mathrm{H}), 7.42-7.46(\mathrm{~m}, 6 \mathrm{H}), 7.61-7.63(\mathrm{~m}$, 3H), 7.72-7.77 (m, 3H); ${ }^{13} \mathrm{C}$ NMR (125 MHz, $\left.\mathrm{CDCl}_{3}\right) \delta$ 
$16.0,55.4,55.8,120.5,124.3,125.4,126.0,126.4,126.5,126.6,126.7,126.9,127.6$, $127.9,128.2,128.3,128.5,128.6,128.7,128.8,129.1,131.5,133.0,134.4,135.9$, 136.2, 139.4, 143.8, 167.0, 169.7; $v$ max $: 1034,1081,1174,1259,1298,1501,1721$, 1757, $3331 \mathrm{~cm}^{-1}$; HRMS (ESI): $\mathrm{m} / z$ calcd for $\mathrm{C}_{35} \mathrm{H}_{29} \mathrm{NNaO}_{3}\left([\mathrm{M}+\mathrm{Na}]^{+}\right)$534.2040, found 534.2029.

$N$-[(1S,2S,E)-2-(Hydroxymethyl)-4-phenyl-1-(4-(trifluoromethyl)phenyl)but-3-en -1-yl]-4-methylbenzenesulfonamide (12)<smiles>OC[C@H](N[TeH])[C@H](/C=C/c1ccccc1)c1ccc(C(F)(F)F)cc1</smiles>

White solid, $63.4 \mathrm{mg}, 89 \%$ yield; mp: $186-187^{\circ} \mathrm{C} ;{ }^{1} \mathrm{H}$ NMR (500 MHz, $\left.\left(\mathrm{CD}_{3}\right)_{2} \mathrm{CO}\right): \delta 2.19(\mathrm{~s}, 3 \mathrm{H}), 2.62(\mathrm{~s}, 1 \mathrm{H})$, $3.28-3.41(\mathrm{~m}, 1 \mathrm{H}), 3.44-3.58(\mathrm{~m}, 1 \mathrm{H}), 3.80(\mathrm{~s}, 1 \mathrm{H}), 4.74$ (t, $J=6.8 \mathrm{~Hz}, 1 \mathrm{H}), 6.06(\mathrm{dd}, J=9.1,15.9 \mathrm{~Hz}, 1 \mathrm{H}), 6.29$ (d, $J=15.9 \mathrm{~Hz}, 1 \mathrm{H}), 6.96-7.27(\mathrm{~m}, 8 \mathrm{H}), 7.36(\mathrm{~s}, 5 \mathrm{H}), 7.69$ $(\mathrm{d}, J=6.7 \mathrm{~Hz}, 1 \mathrm{H}) ;{ }^{13} \mathrm{C}$ NMR $\left(125 \mathrm{MHz},\left(\mathrm{CD}_{3}\right)_{2} \mathrm{CO}\right): \delta$ 20.1, 51.5, 58.1, 62.2, 124.4, 125.9, 126.5, 126.9, 127.9, 128.1, 128.8, 133.2, 137.1, 138, 138.8, 142.2, 145.1; $v_{\max }$ : 1017, 1068, 1093, 1121, 1159, 1196, 1325, 1495, 1597, 1619, $3519 \mathrm{~cm}^{-1}$; HRMS $(\mathrm{m} / z)$ calcd for $\mathrm{C}_{25} \mathrm{H}_{24} \mathrm{~F}_{3} \mathrm{NNaO}_{3} \mathrm{~S}\left([\mathrm{M}+\mathrm{Na}]^{+}\right)$: 498.1321; found: 498.1312 .

(2R,3S,4S)-3-Bromo-2-phenyl-4-\{(S)-(4-toluenesulfonamido)-[4-(trifluoromethyl) phenyl]methyl\}tetrahydrofuran (13)

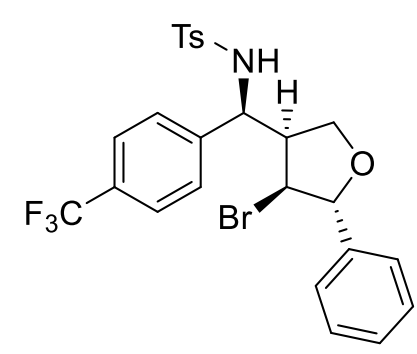

White solid, $47 \mathrm{mg}, 85 \%$ yield; $\mathrm{mp}: 209-211^{\circ} \mathrm{C} ;[\alpha]_{\mathrm{D}}^{25}=$ -124 (c 1.0, $\mathrm{CHCl}_{3},>99: 1 \mathrm{dr},>99 \%$ ee) ${ }^{1} \mathrm{H}$ NMR (500 $\left.\mathrm{MHz}, \mathrm{CDCl}_{3}\right): \delta 2.34(\mathrm{~s}, 3 \mathrm{H}), 2.71-2.75(\mathrm{~m}, 1 \mathrm{H}), 3.56$ $(\mathrm{dd}, J=4.4,11.0 \mathrm{~Hz}, 1 \mathrm{H}), 3.76(\mathrm{dd}, J=4.8,11.0 \mathrm{~Hz}, 1 \mathrm{H})$, $4.45(\mathrm{t}, J=7.2 \mathrm{~Hz}, 1 \mathrm{H}), 5.13(\mathrm{~d}, J=7.4 \mathrm{~Hz}, 1 \mathrm{H}), 5.27(\mathrm{~d}$, $J=6.8 \mathrm{~Hz}, 1 \mathrm{H}), 6.96(\mathrm{q}, J=8.3 \mathrm{~Hz}, 4 \mathrm{H}), 7.27-7.46(\mathrm{~m}, 9 \mathrm{H}) ;{ }^{13} \mathrm{C} \mathrm{NMR}(125 \mathrm{MHz}$, $\left.\mathrm{CDCl}_{3}\right): \delta 21.3,52.5,59.4,66,74.3,125.2,125.2,126.8,128.2,128.4,128.5,128.8$, 128.9, 137.1, 138.3, 142.7, 143.6; v $\max : 1045,1069,1094,1116,1156,1196,1326$, 1474, 1495, 1598, 1618, 3047, $3579 \mathrm{~cm}^{-1}$; Enantiomeric excess of 12 was determined by chiral stationary phase HPLC analysis using a ChiralPak IC column (80: 20 
hexane $/ i-\mathrm{PrOH}$ at $1.0 \mathrm{~mL} / \mathrm{min}, \lambda=254 \mathrm{~nm}$ ), major enantiomer: $\mathrm{t}_{\mathrm{R}}=6.34 \mathrm{~min}$, minor enantiomer: $t_{R}=8.7 \mathrm{~min}$; HRMS $(\mathrm{m} / \mathrm{z})$ calcd for $\mathrm{C}_{25} \mathrm{H}_{23} \mathrm{BrF}_{3} \mathrm{NNaO}_{3} \mathrm{~S}\left([\mathrm{M}+\mathrm{Na}]^{+}\right)$: 576.0426; found: 576.0437 . 


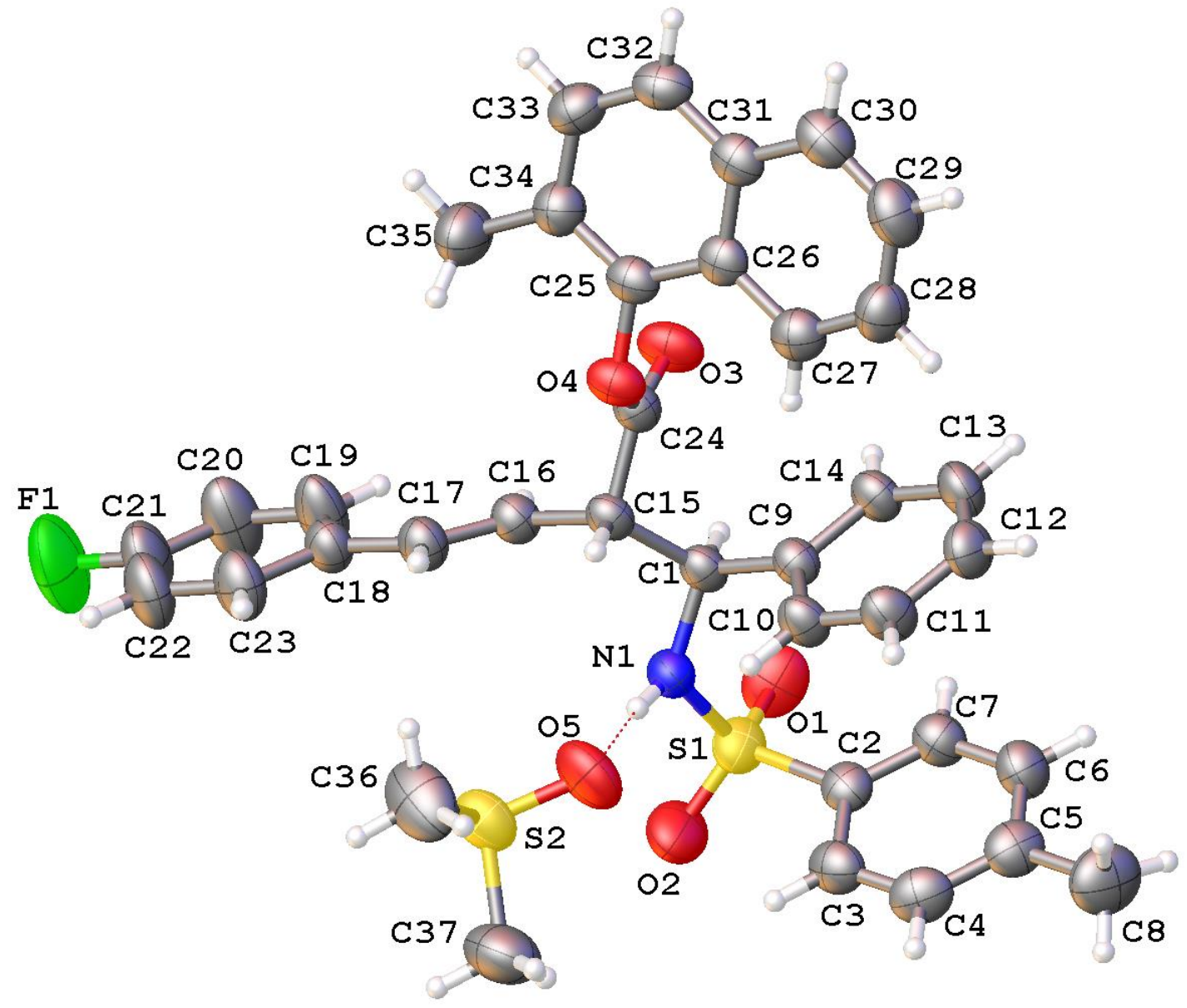

Figure S-1. ORTEP Drawing of 9p (DMSO solveate) [CCDC No. 1408220] 


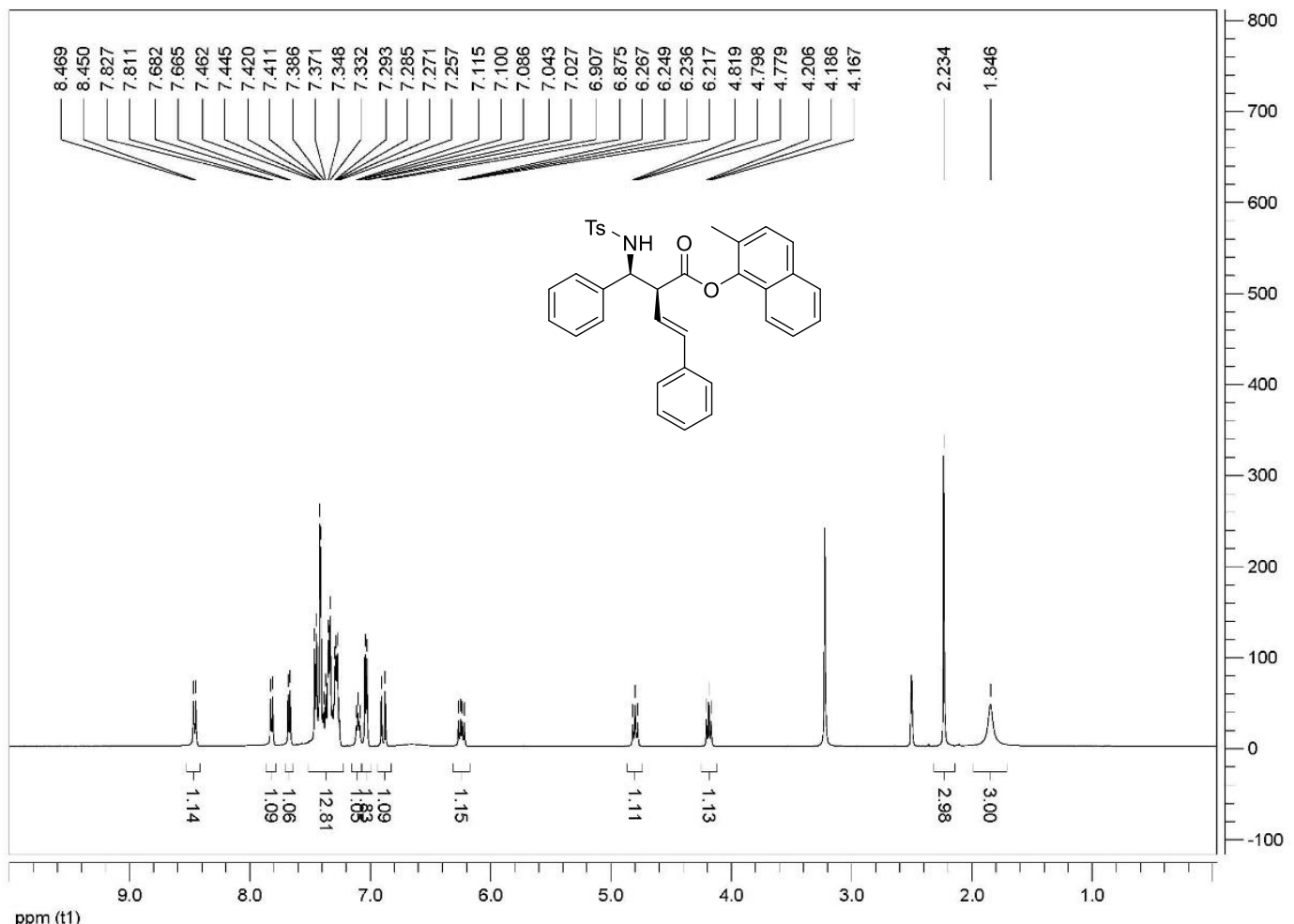

${ }^{1} \mathrm{H}$ NMR of 9d

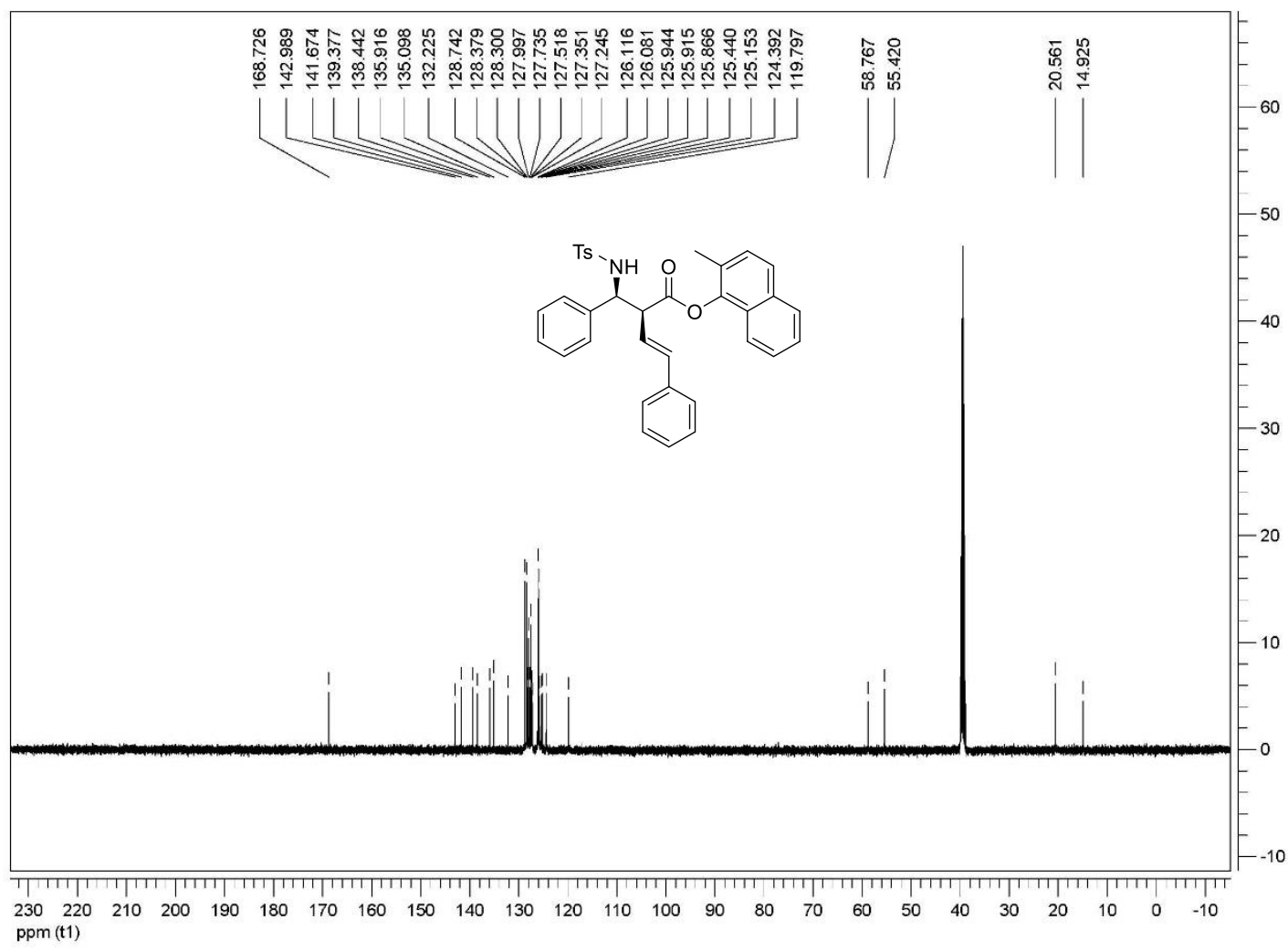

${ }^{13} \mathrm{C}$ NMR of 9d 

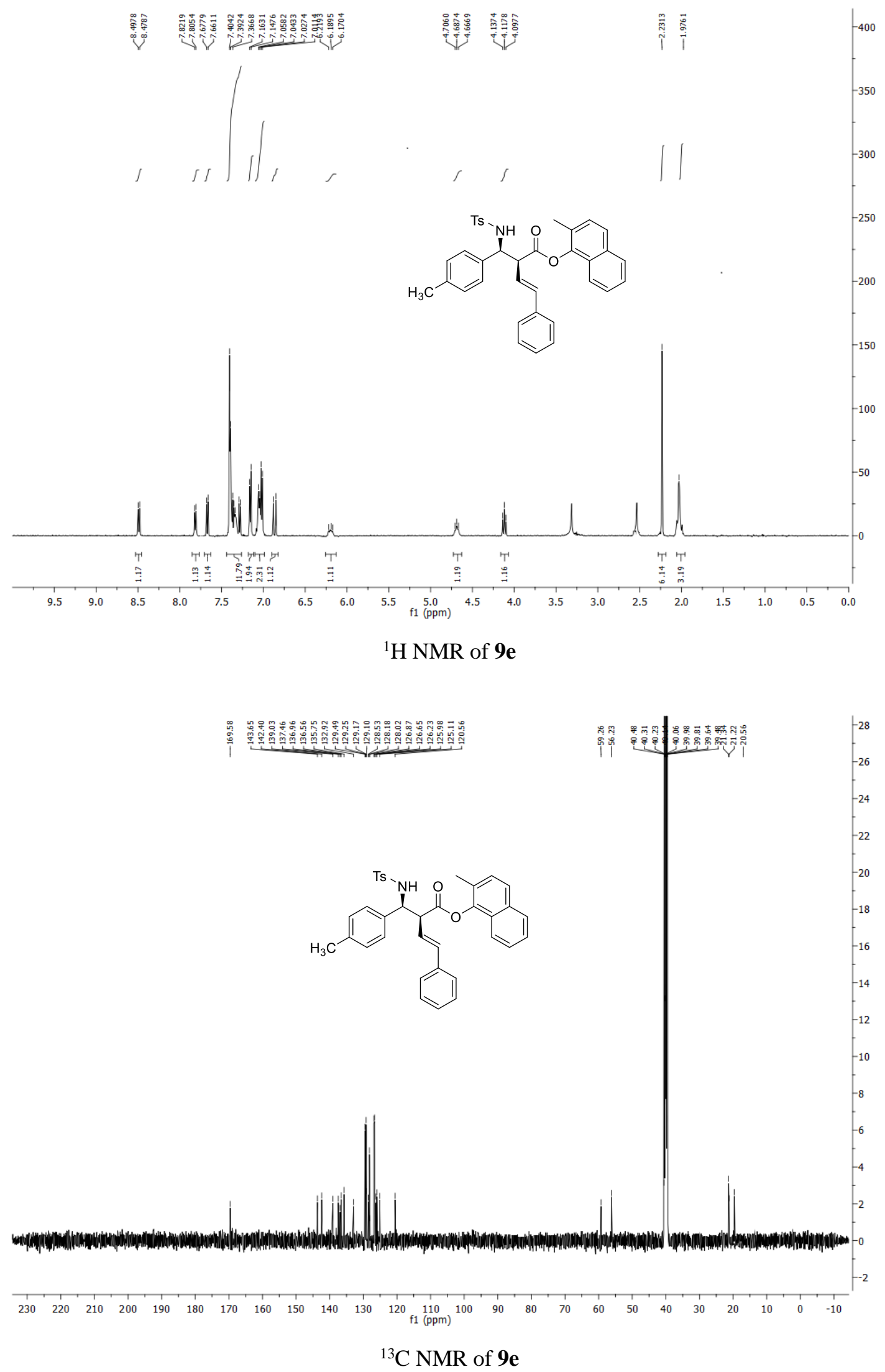
<smiles>CNC(c1ccc(OC)cc1)C(C(=O)OCc1ccccc1)C(=O)Oc1c(C)ccc2ccccc12</smiles>
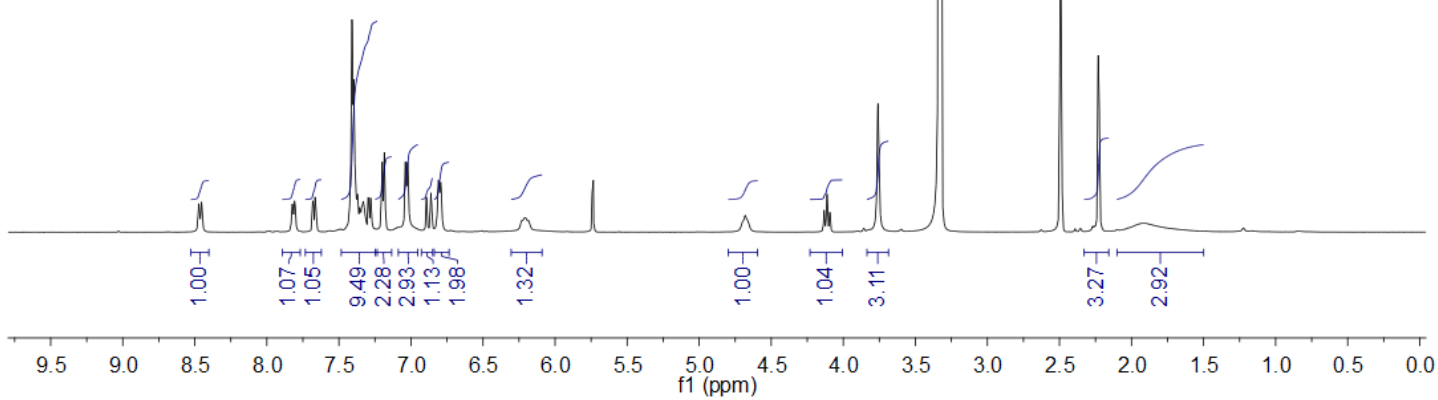

${ }^{1} \mathrm{H}$ NMR of $9 \mathbf{f}$

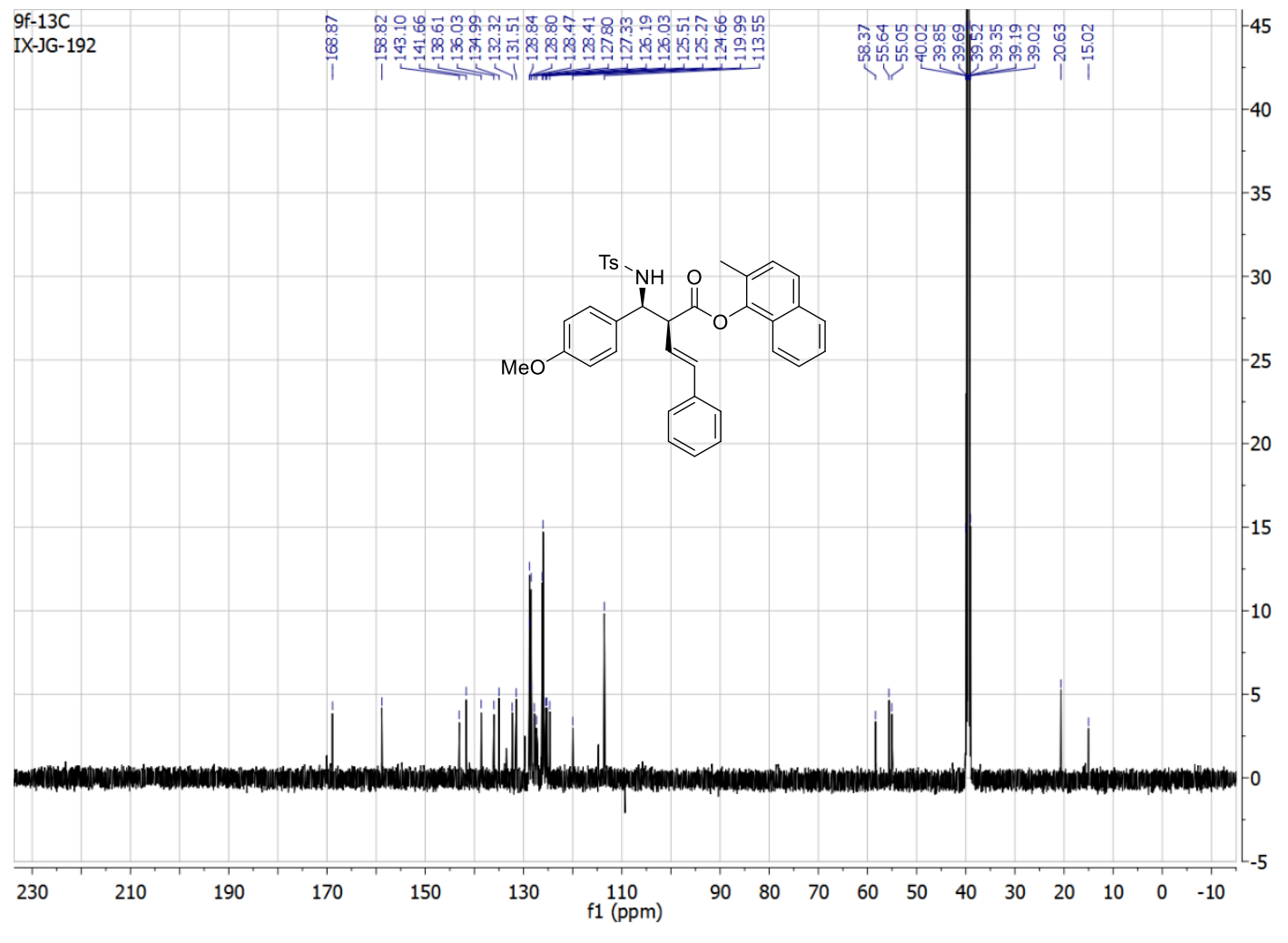

${ }^{13} \mathrm{C}$ NMR of 9f 


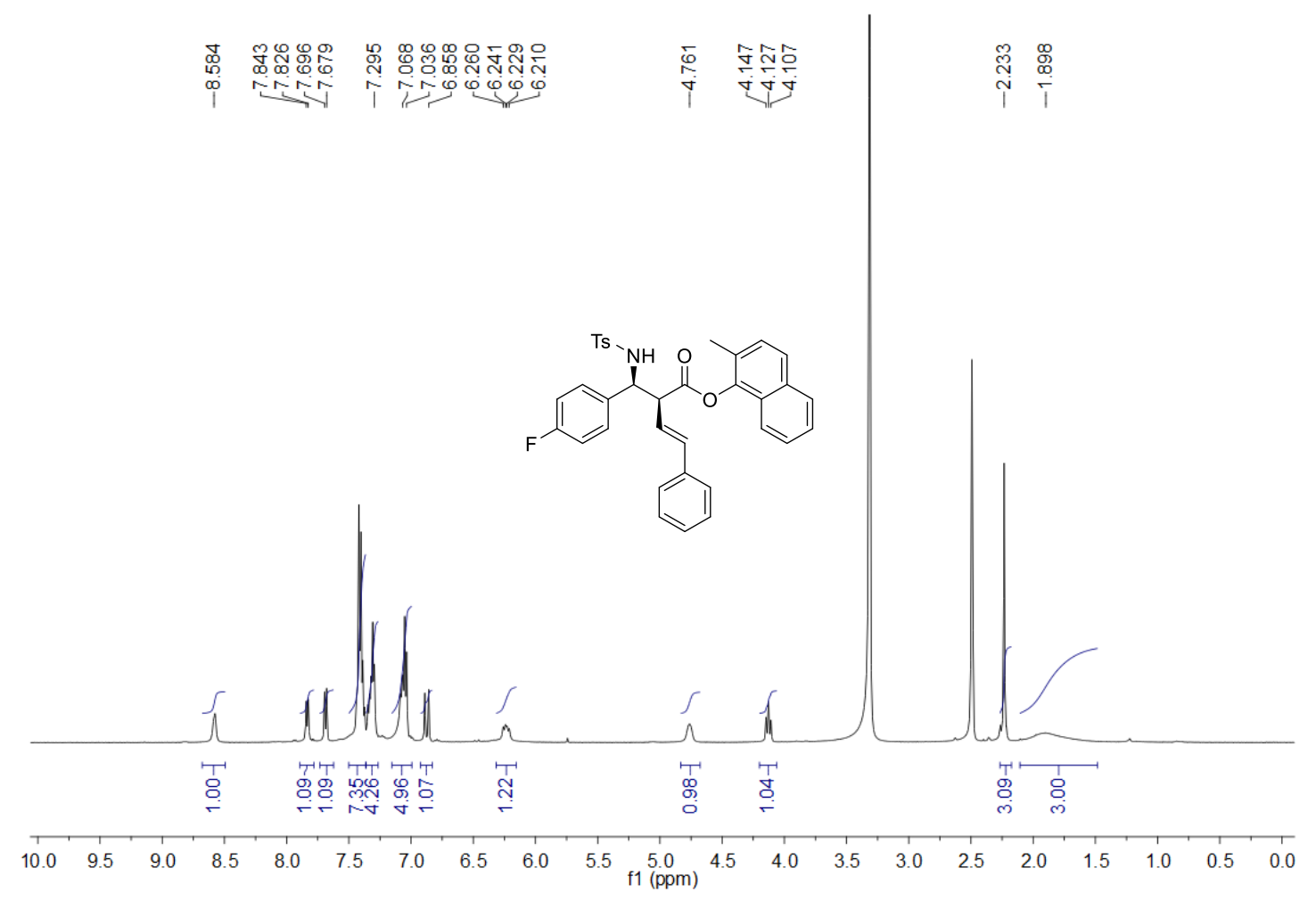

${ }^{1} \mathrm{H}$ NMR of $9 \mathrm{~g}$

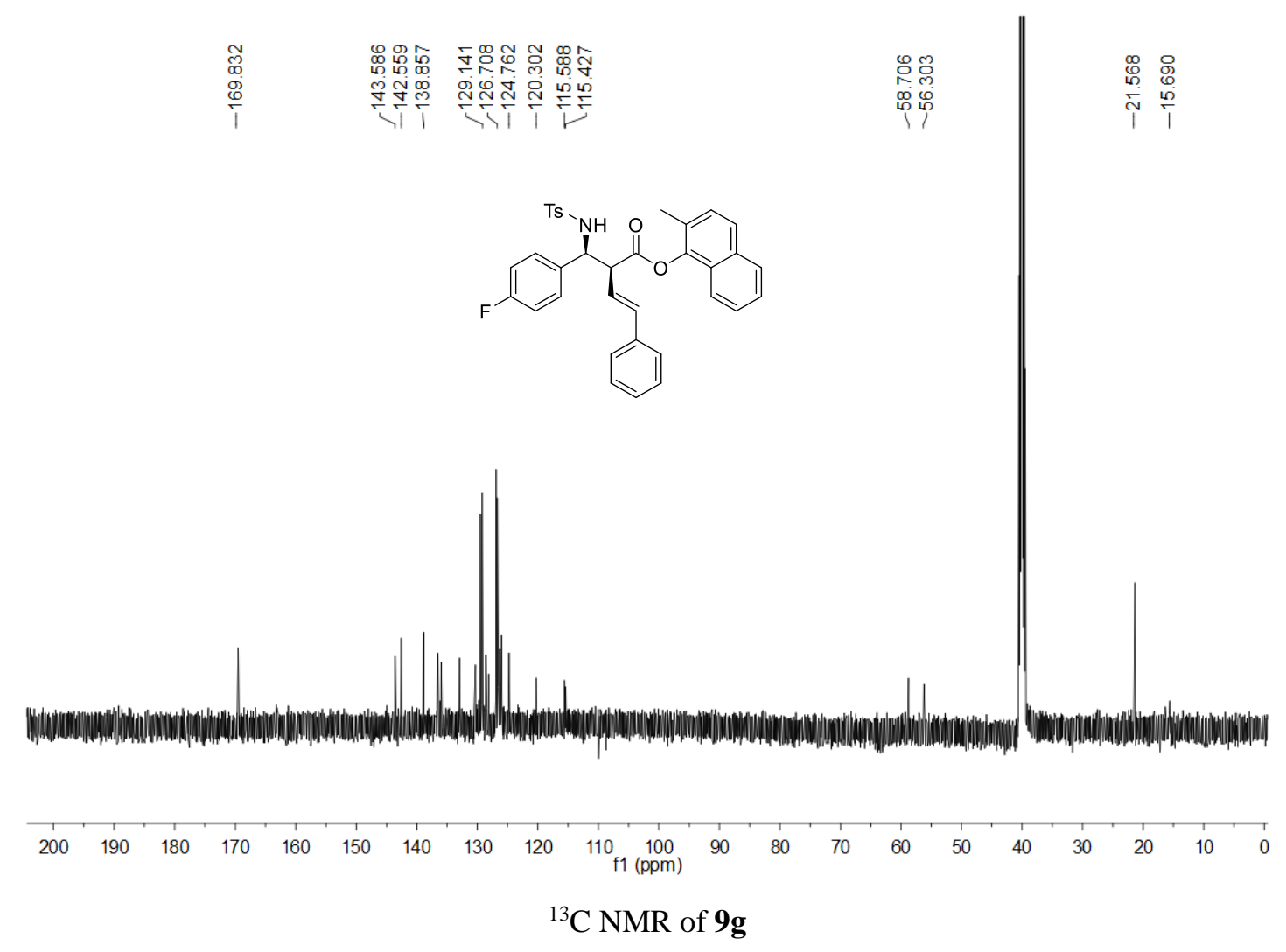




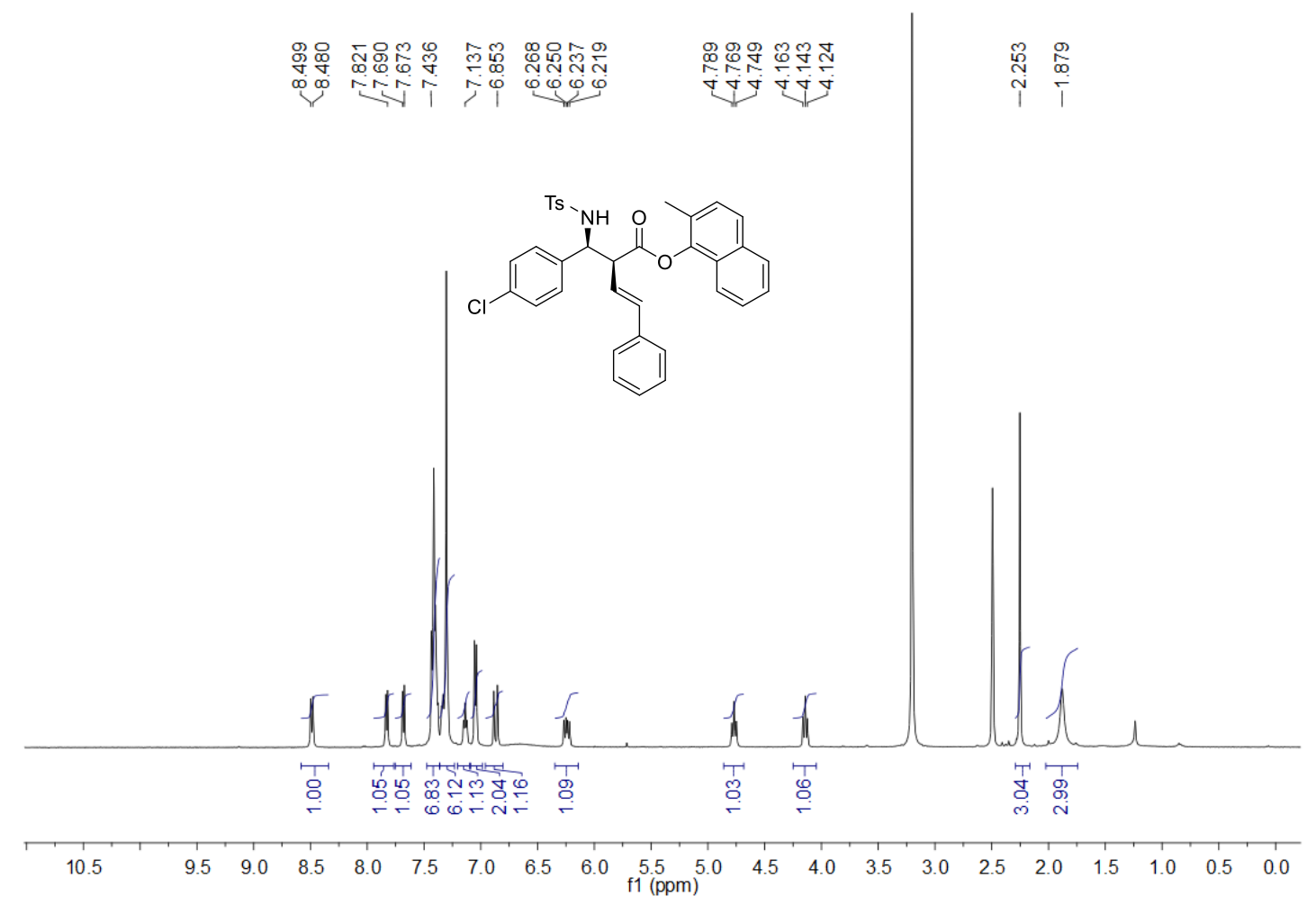

${ }^{1} \mathrm{H}$ NMR of $\mathbf{9 h}$

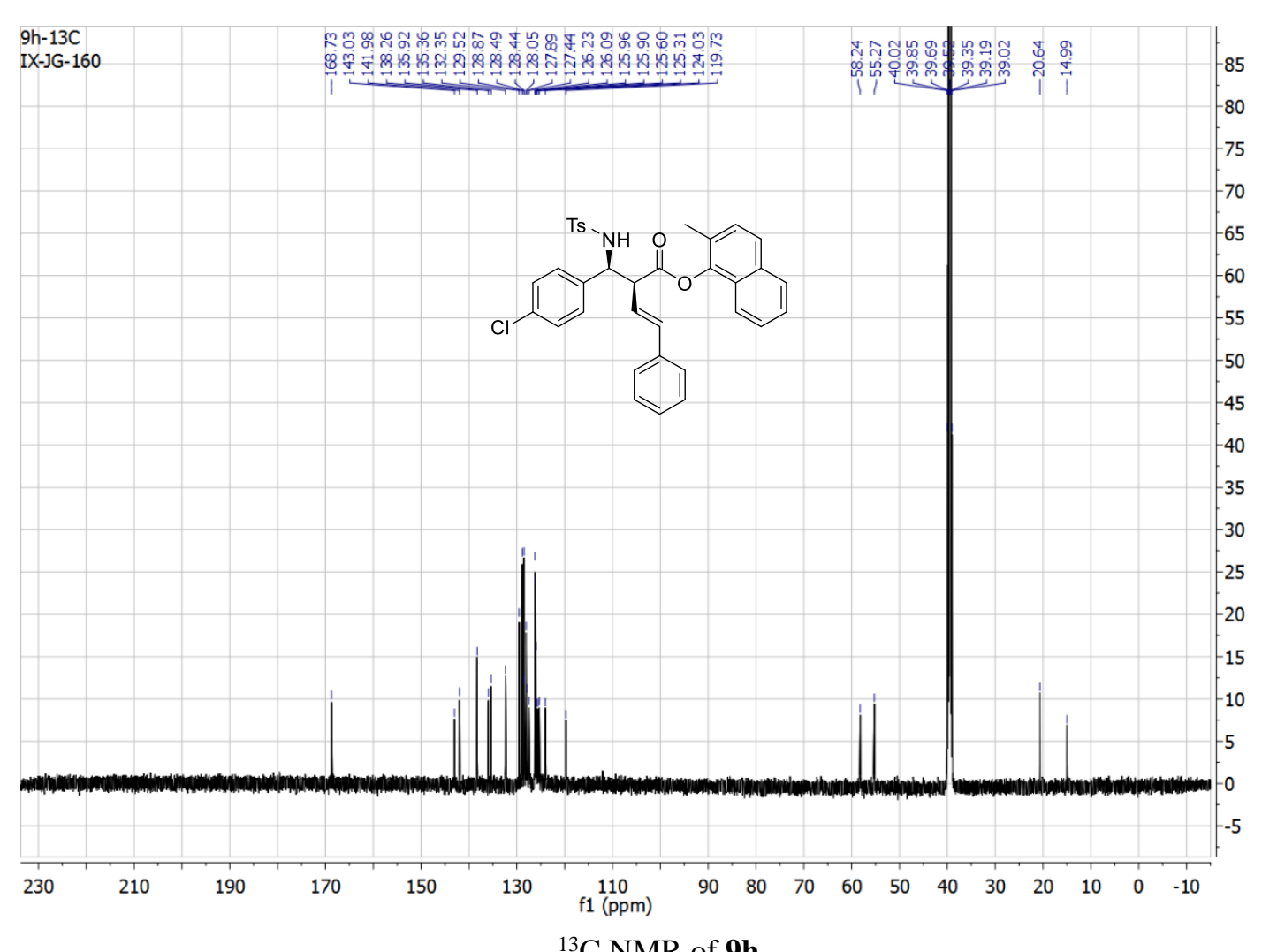

${ }^{13} \mathrm{C}$ NMR of $9 h$ 


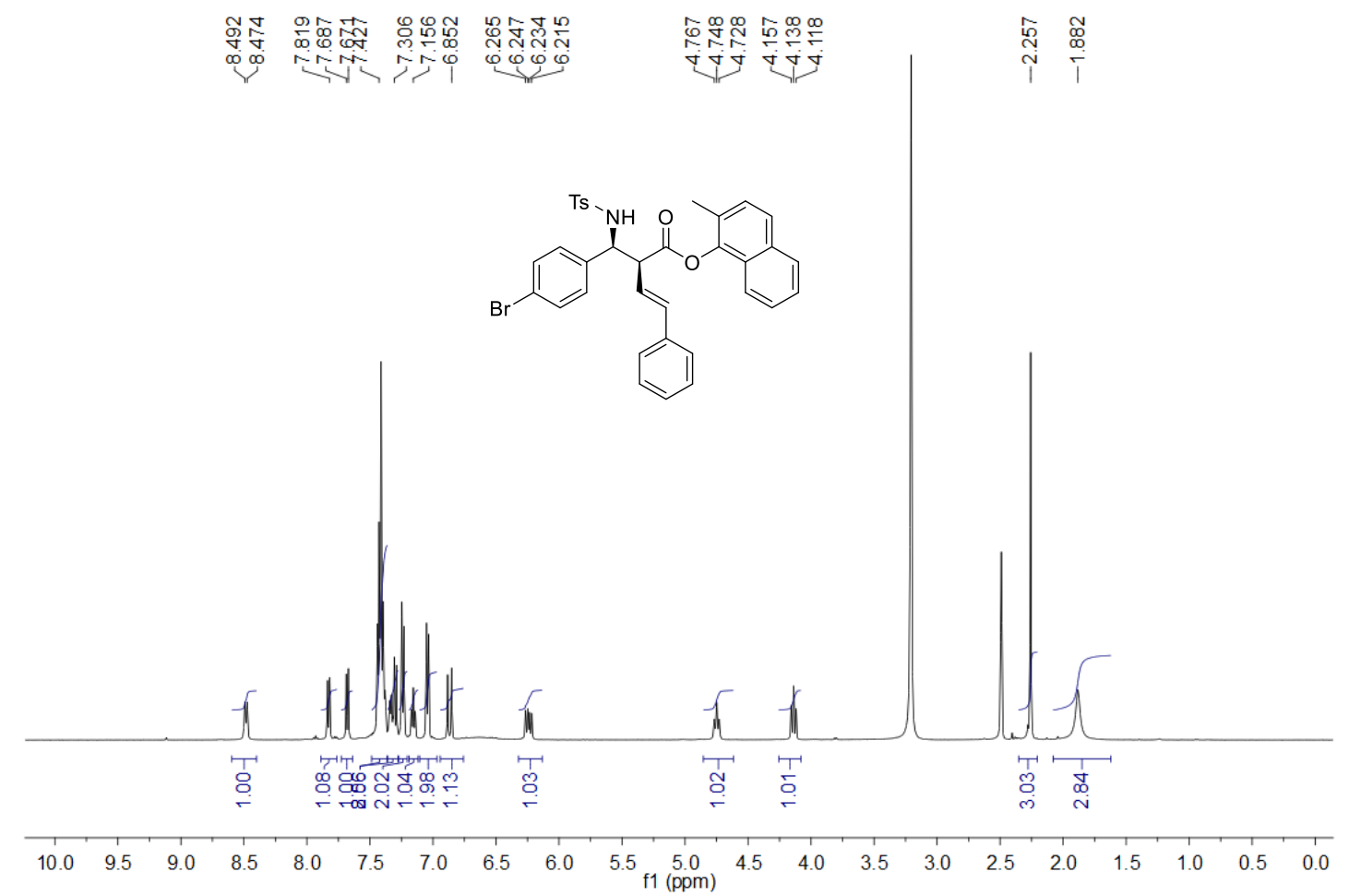

${ }^{1} \mathrm{H}$ NMR of $9 \mathbf{i}$

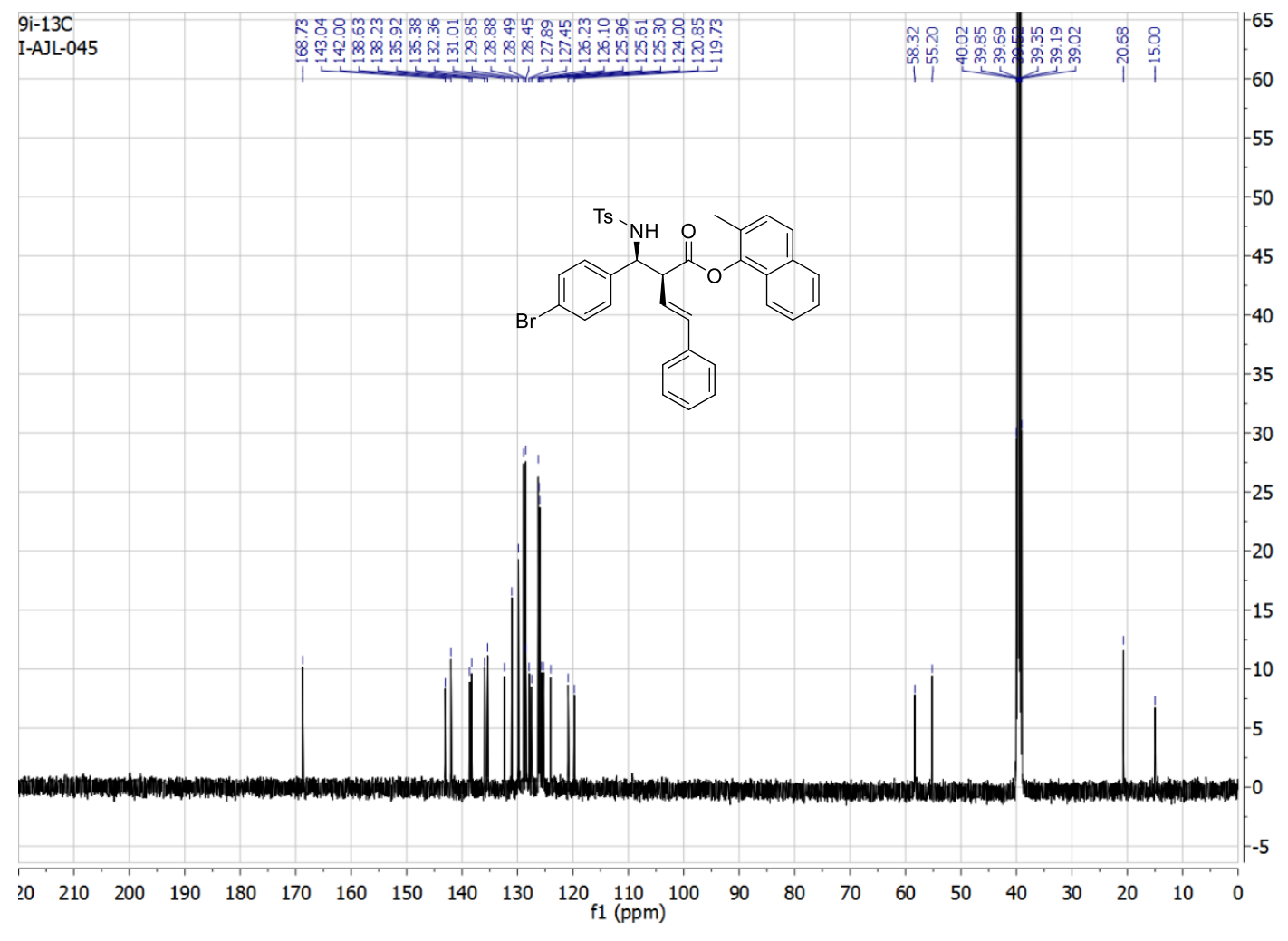

${ }^{13} \mathrm{C}$ NMR of $9 \mathbf{i}$ 


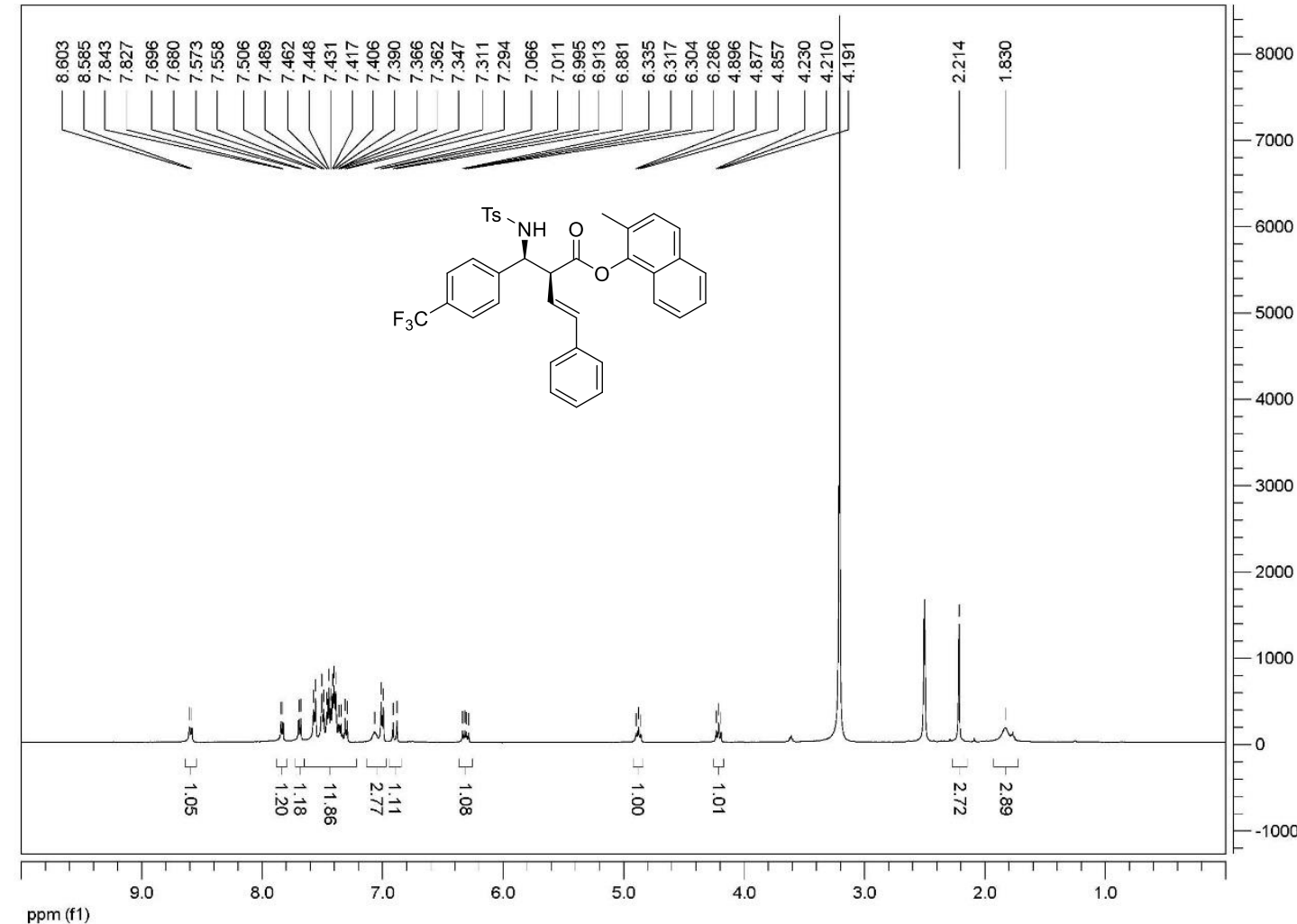

${ }^{1} \mathrm{H}$ NMR of $\mathbf{9 j}$

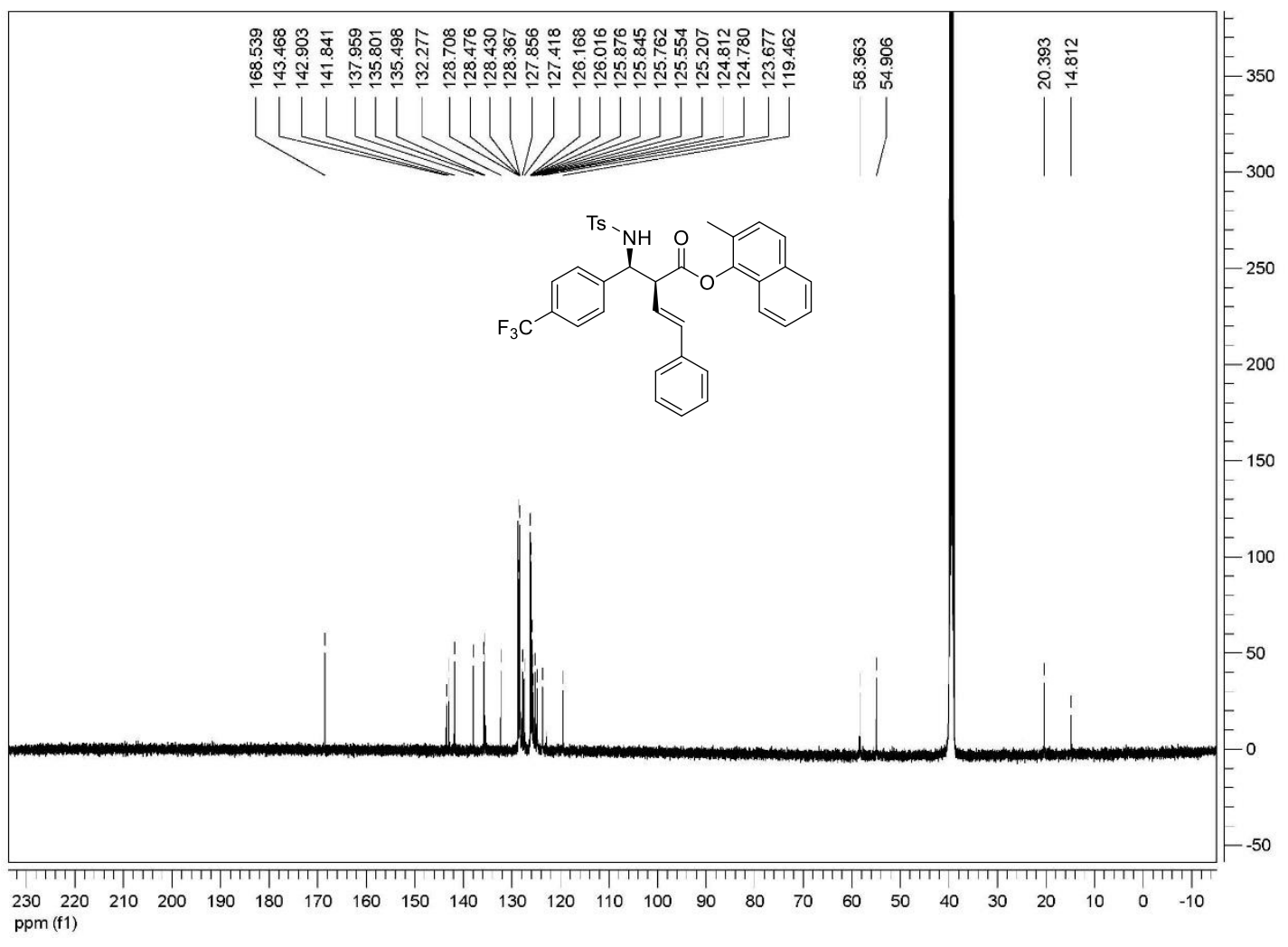

${ }^{13}$ C NMR of $9 j$ 


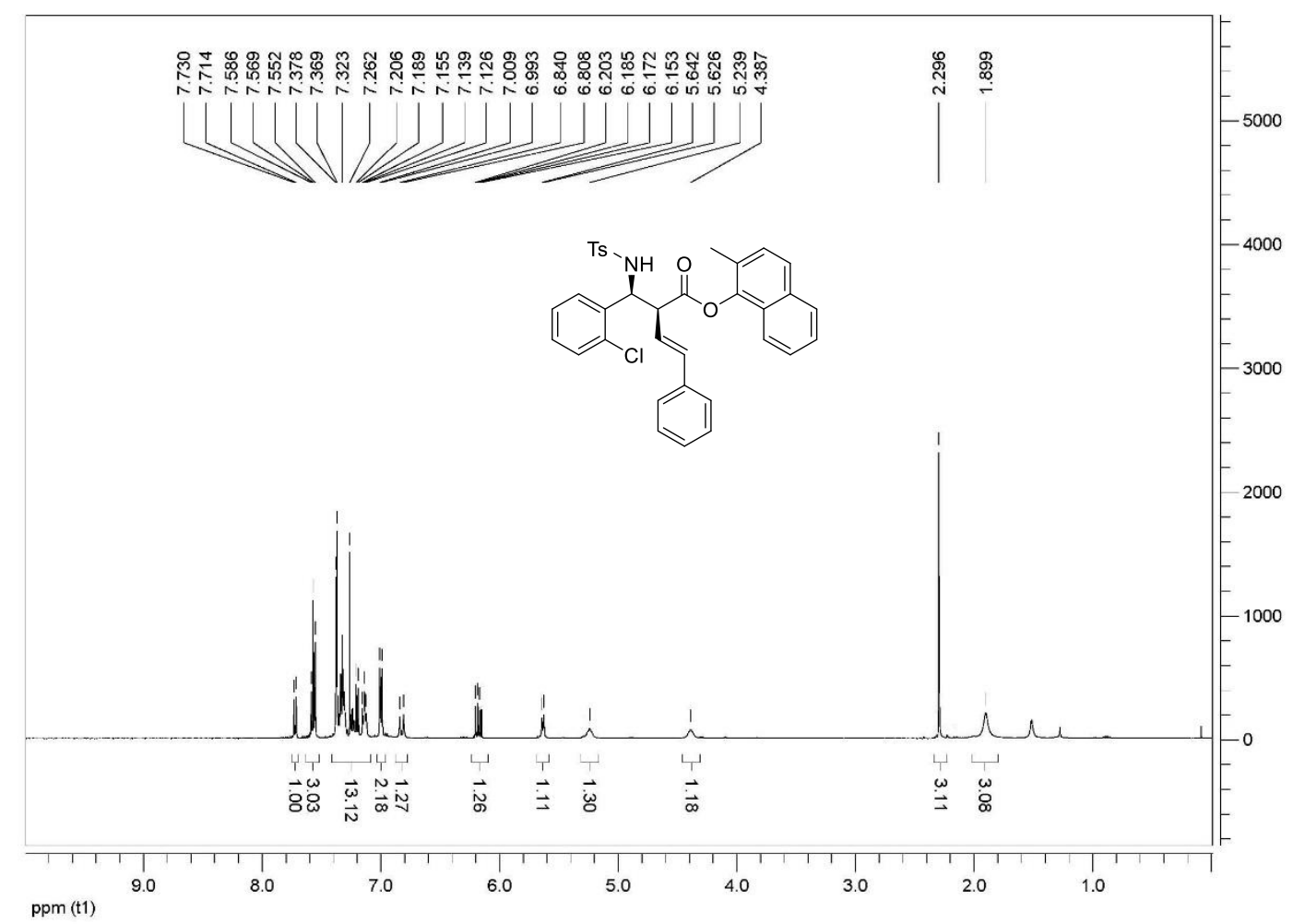

${ }^{1} \mathrm{H}$ NMR of $9 k$

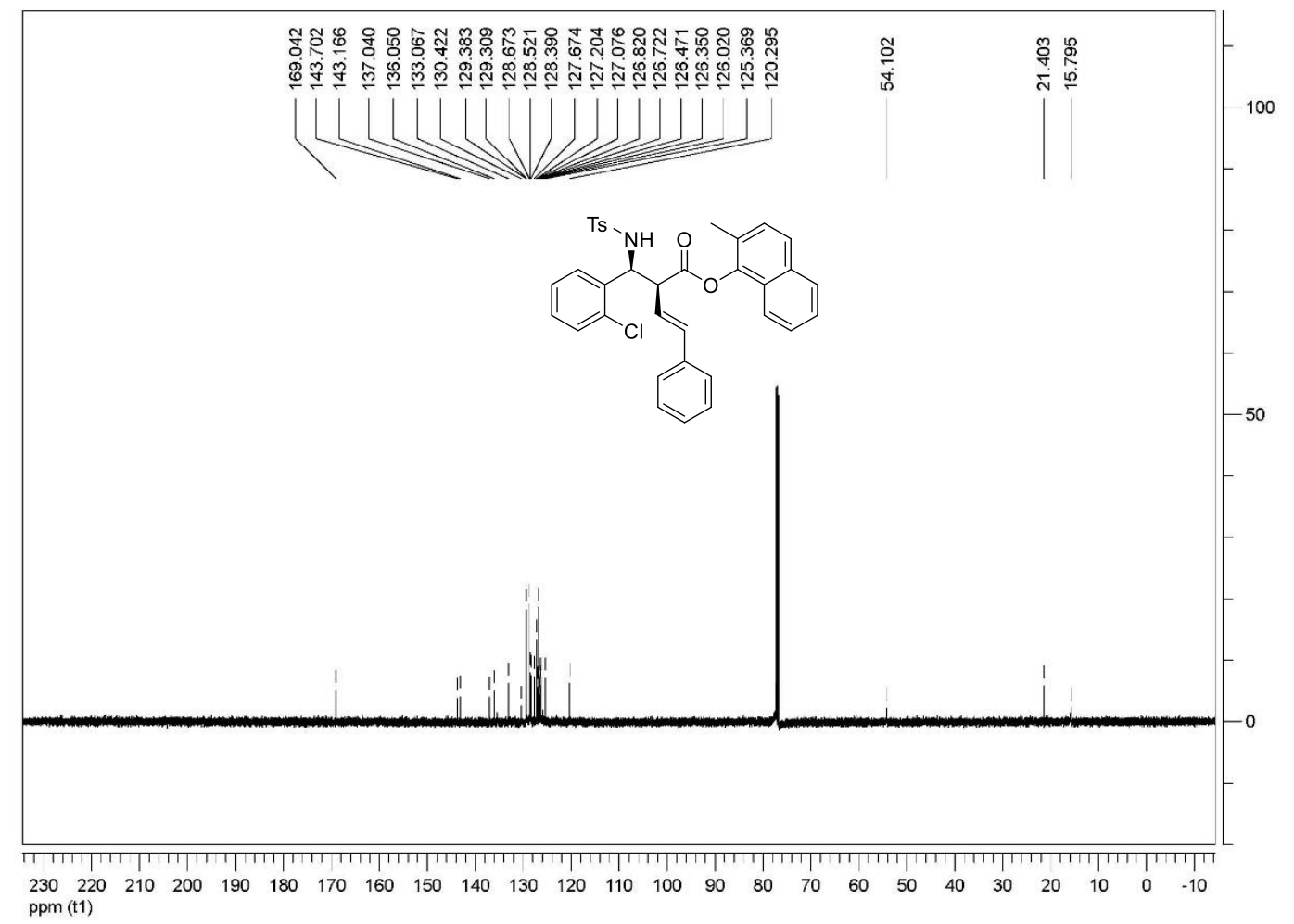

${ }^{13} \mathrm{C}$ NMR of $9 k$ 


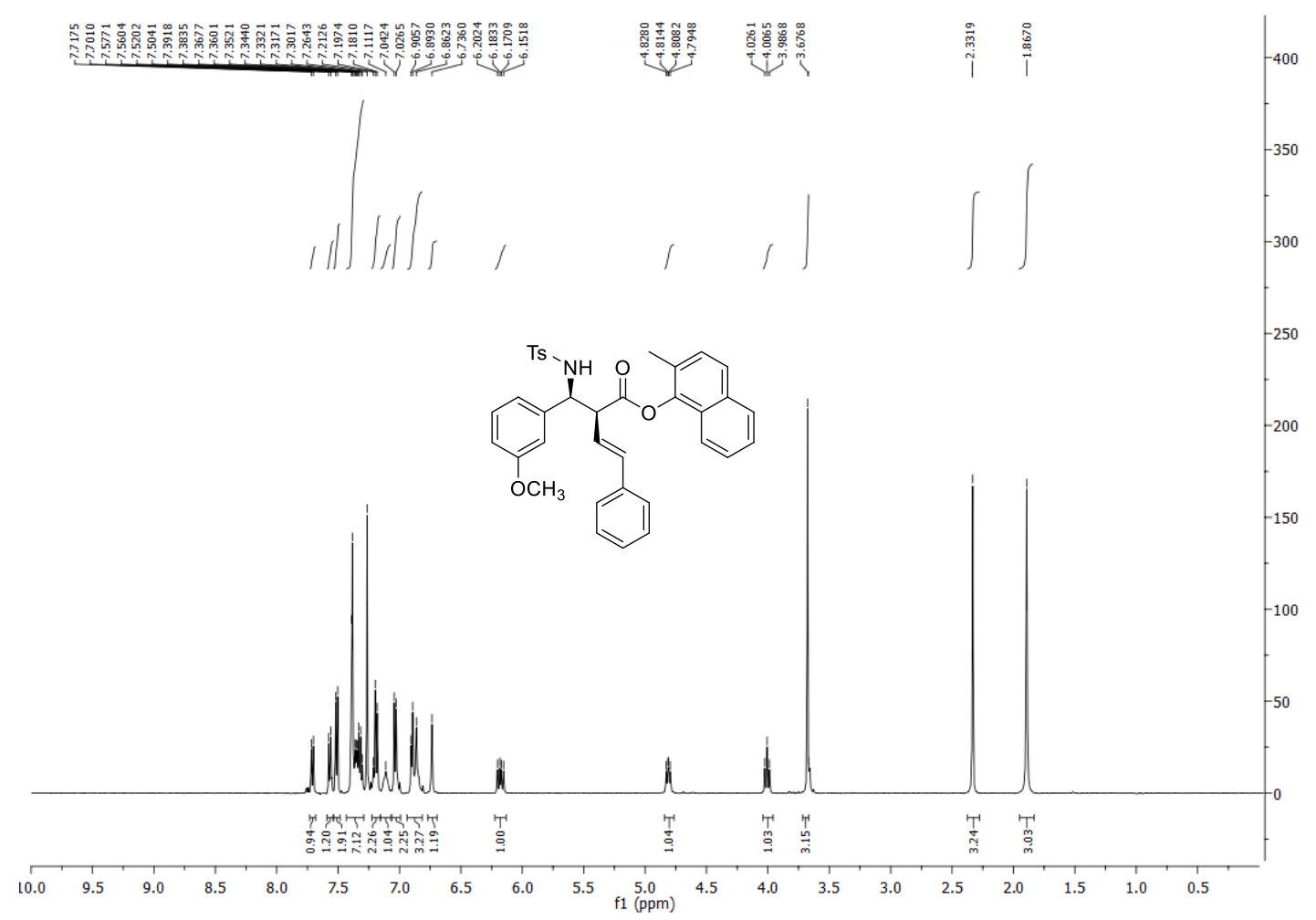

${ }^{1} \mathrm{H}$ NMR of 91

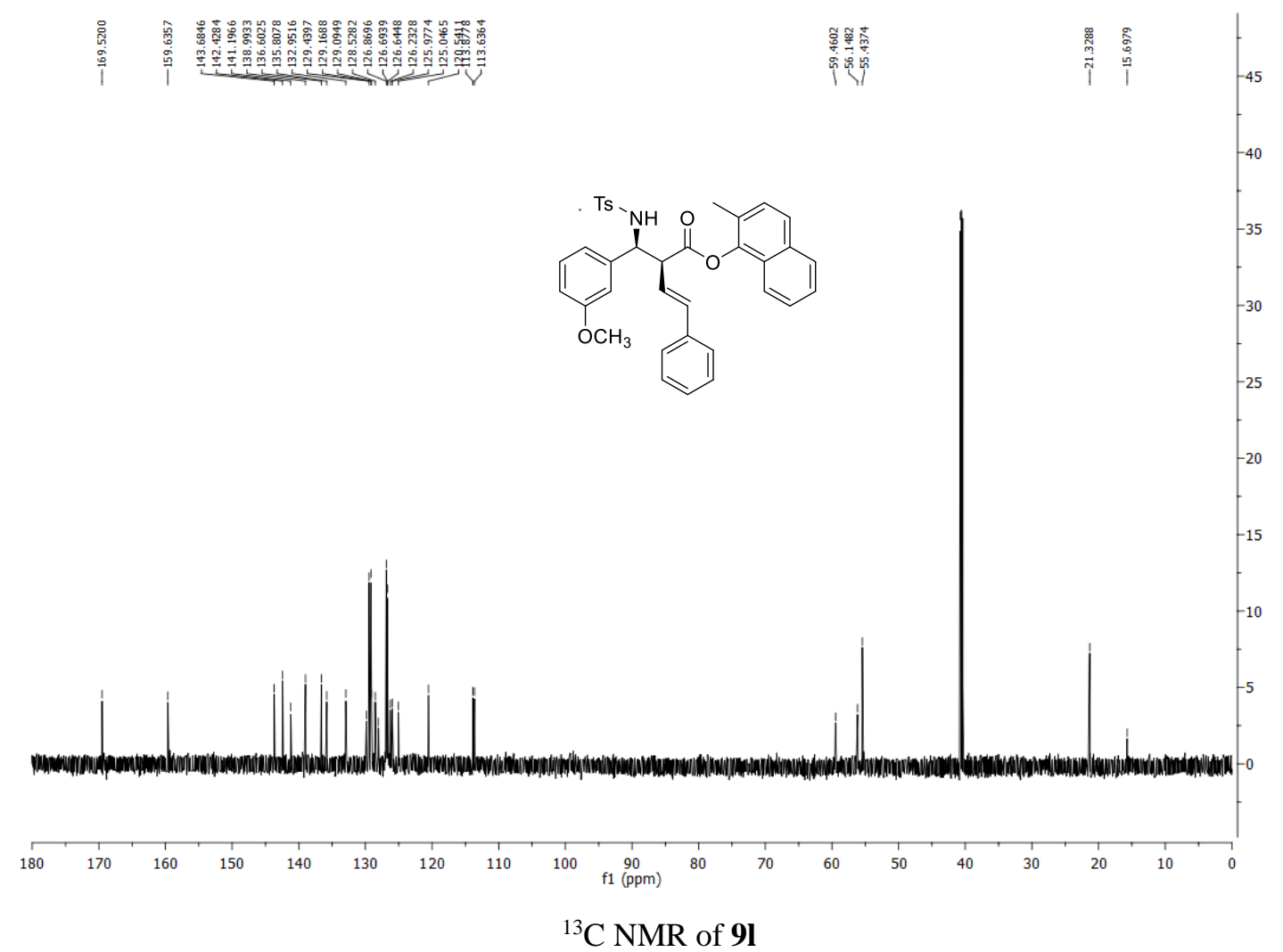




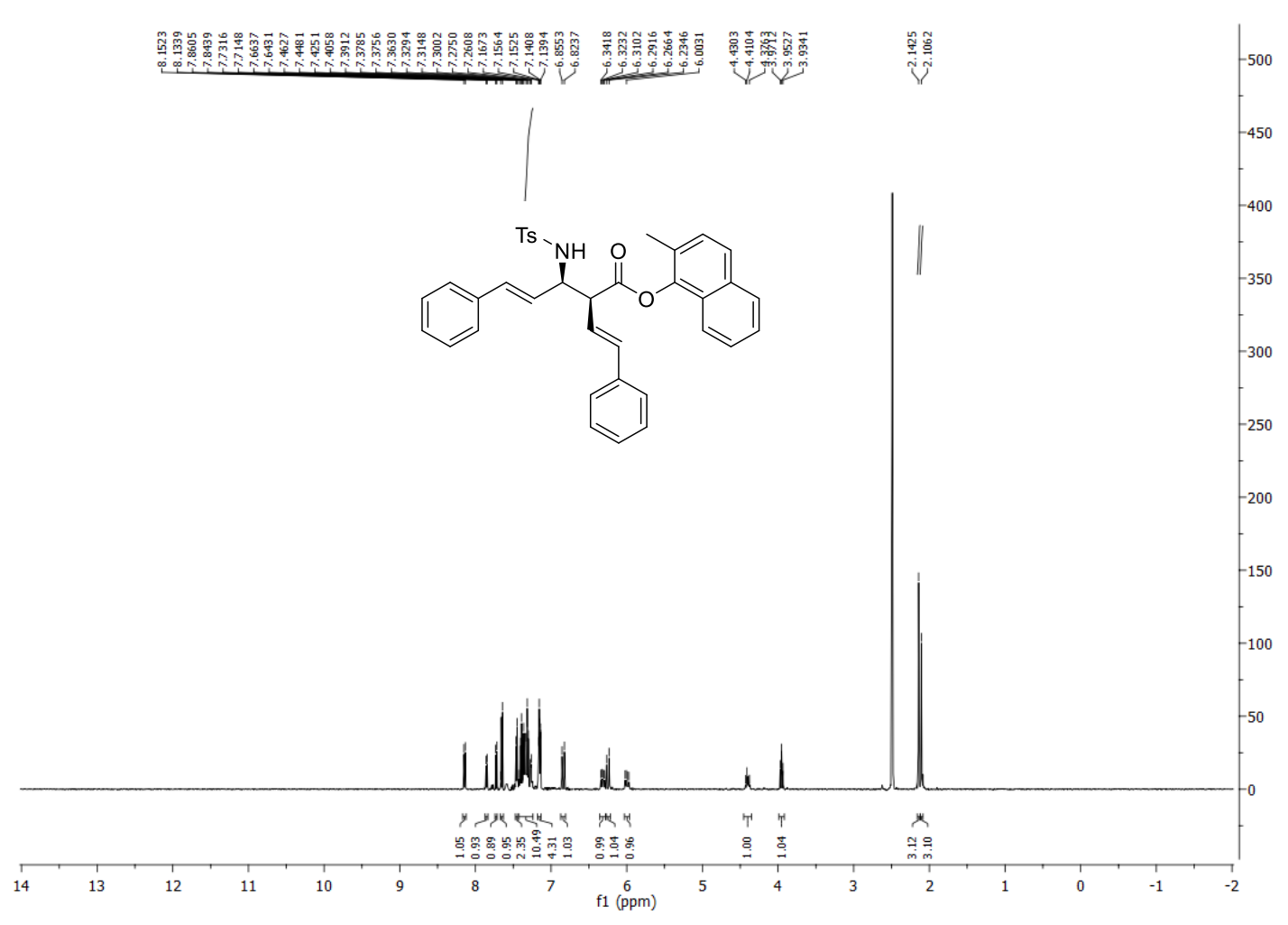

${ }^{1} \mathrm{H}$ NMR of $\mathbf{9 m}$

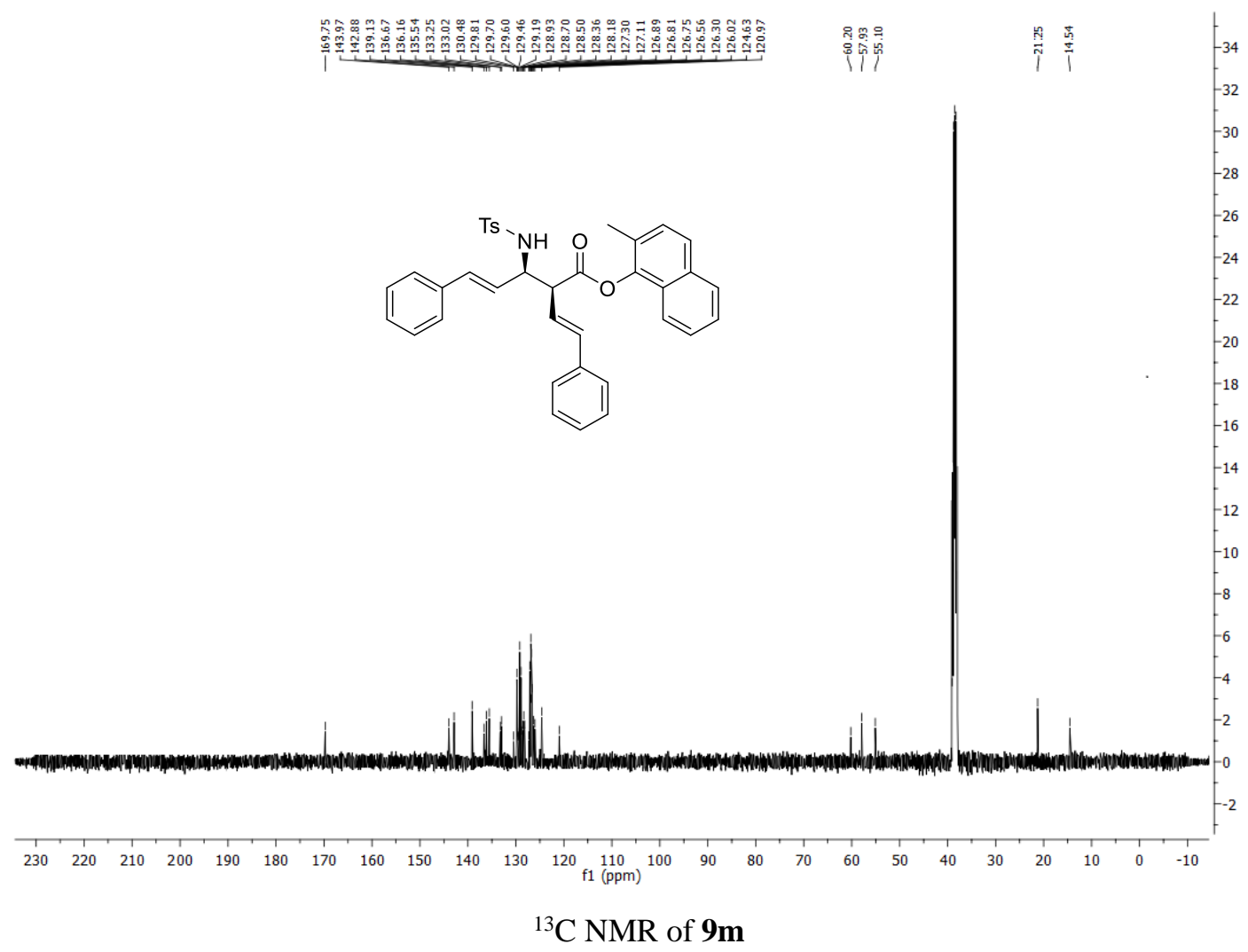




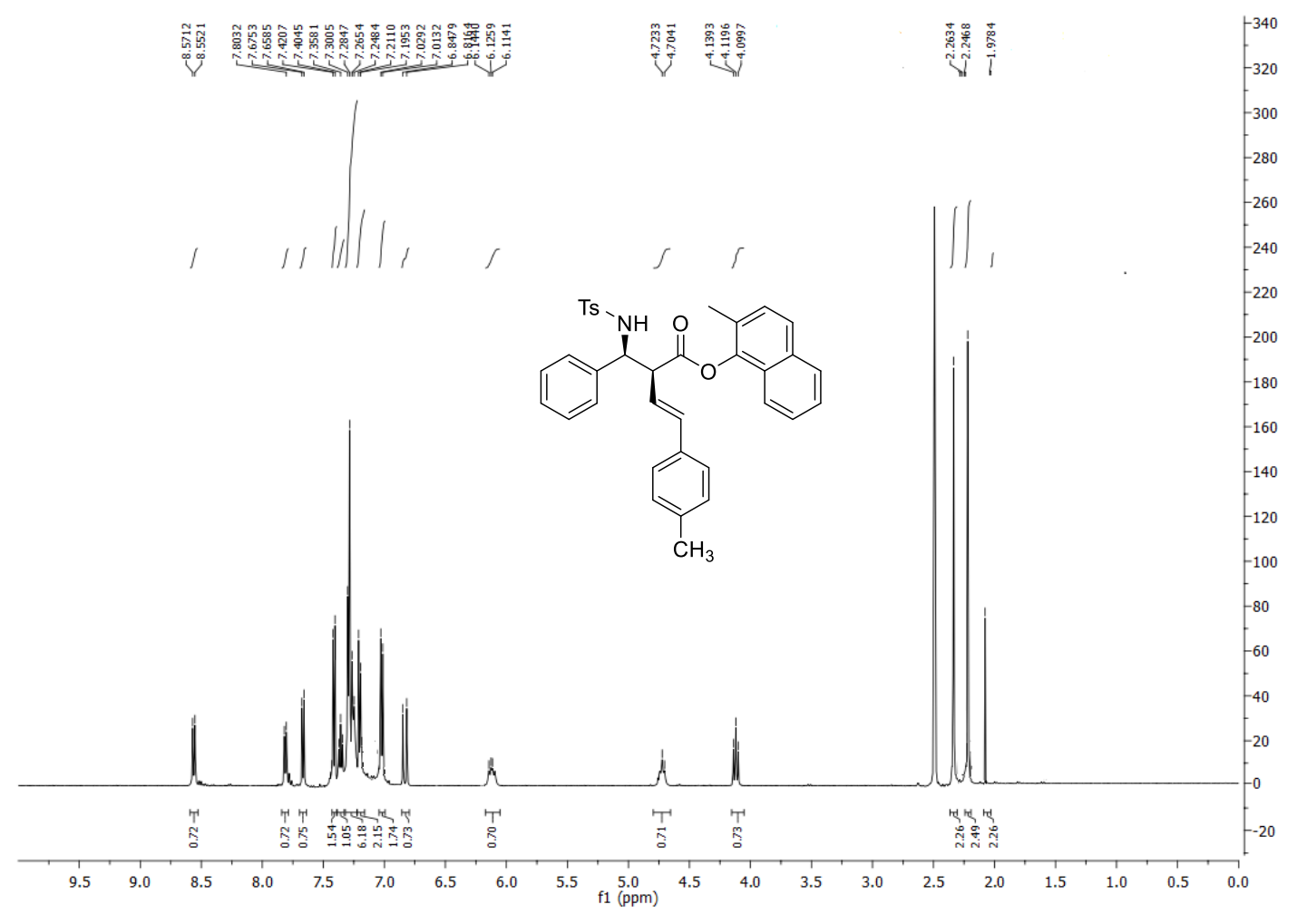

${ }^{1} \mathrm{H}$ NMR of 9n

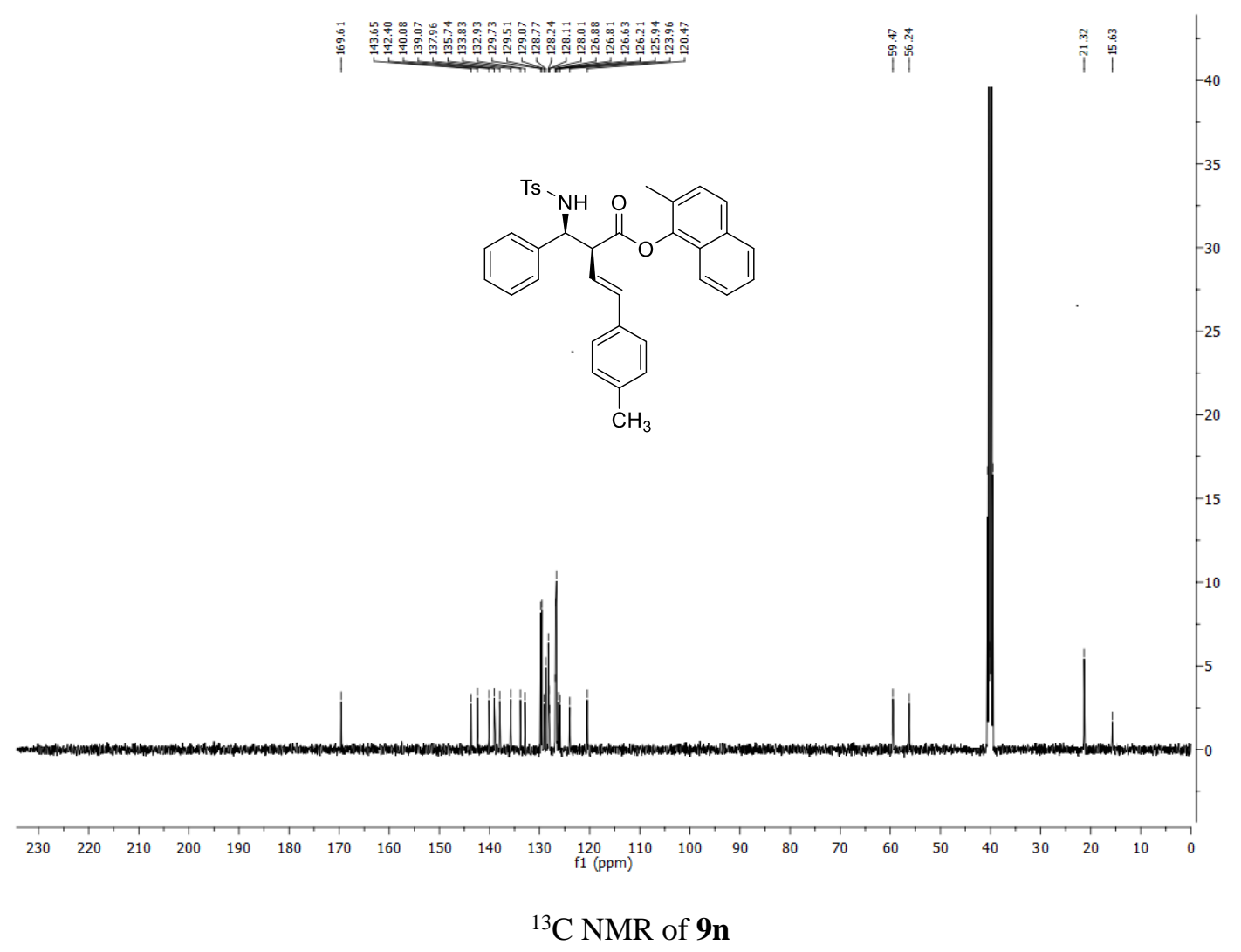




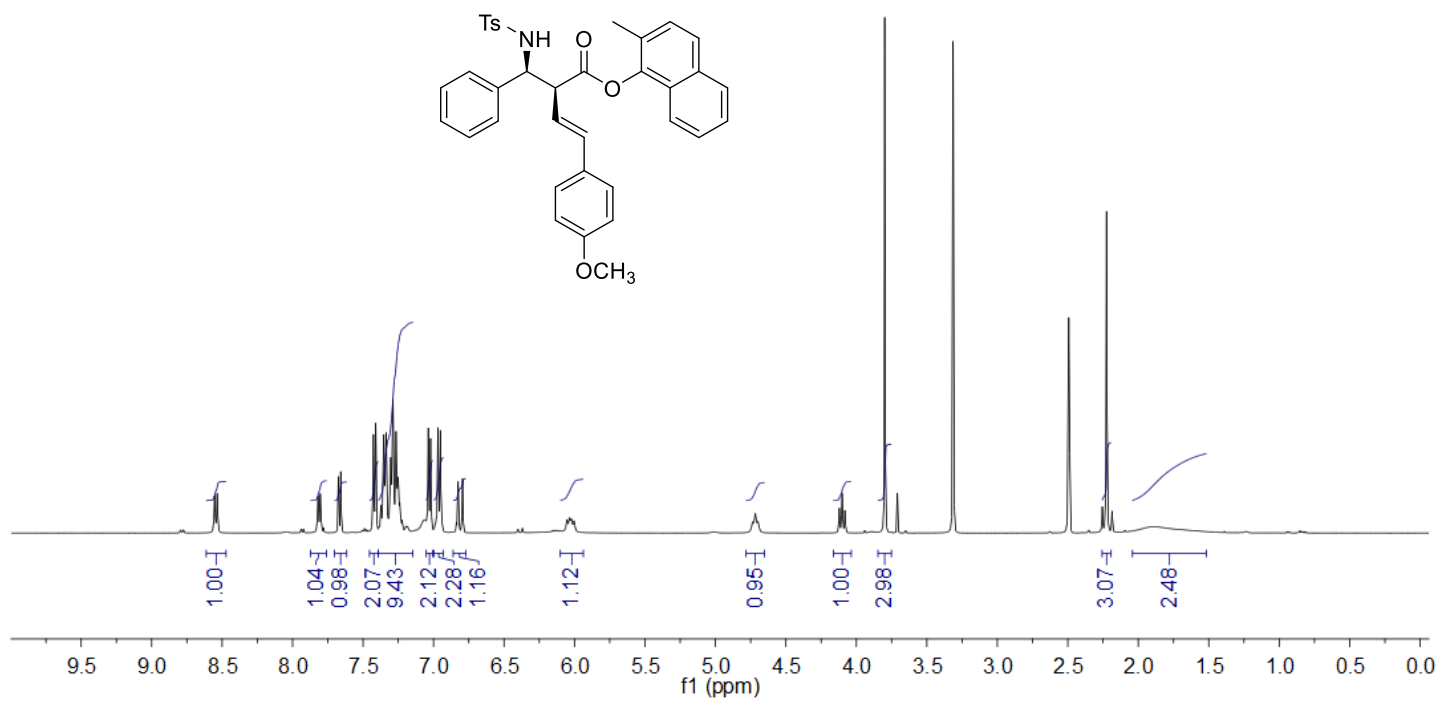

${ }^{1} \mathrm{H}$ NMR of 90

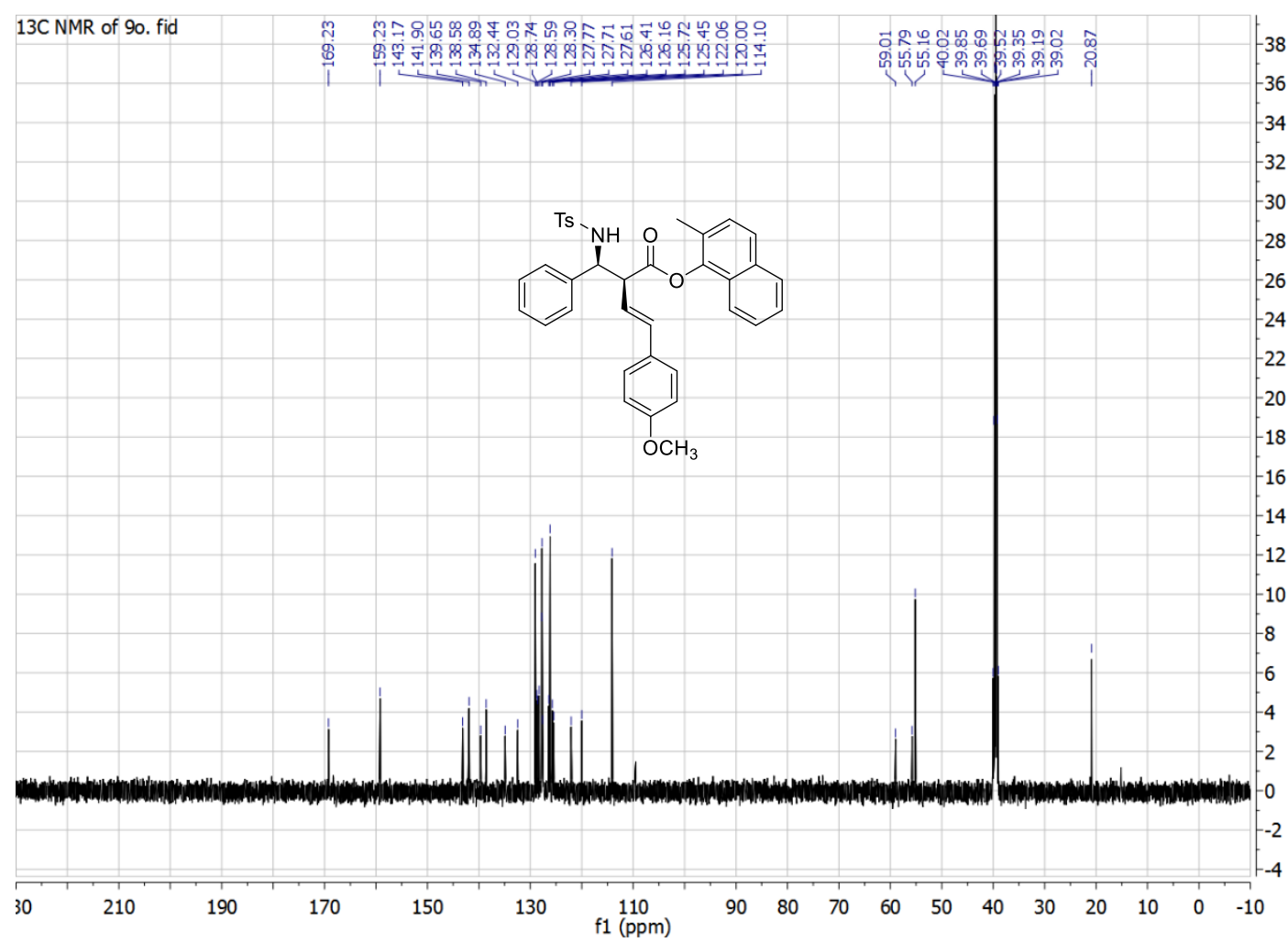

${ }^{13} \mathrm{C}$ NMR of 90 


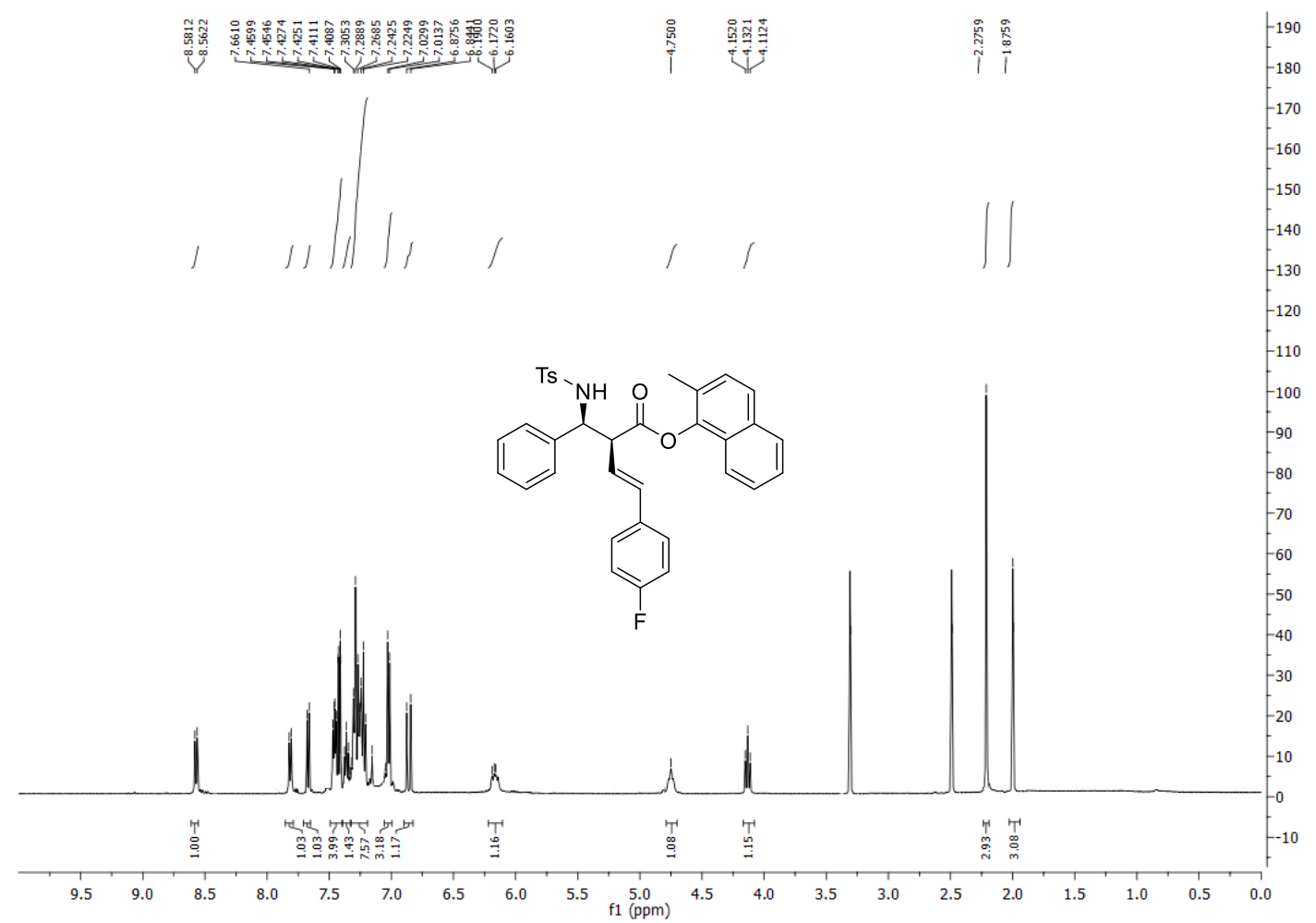

${ }^{1} \mathrm{H}$ NMR of 9p

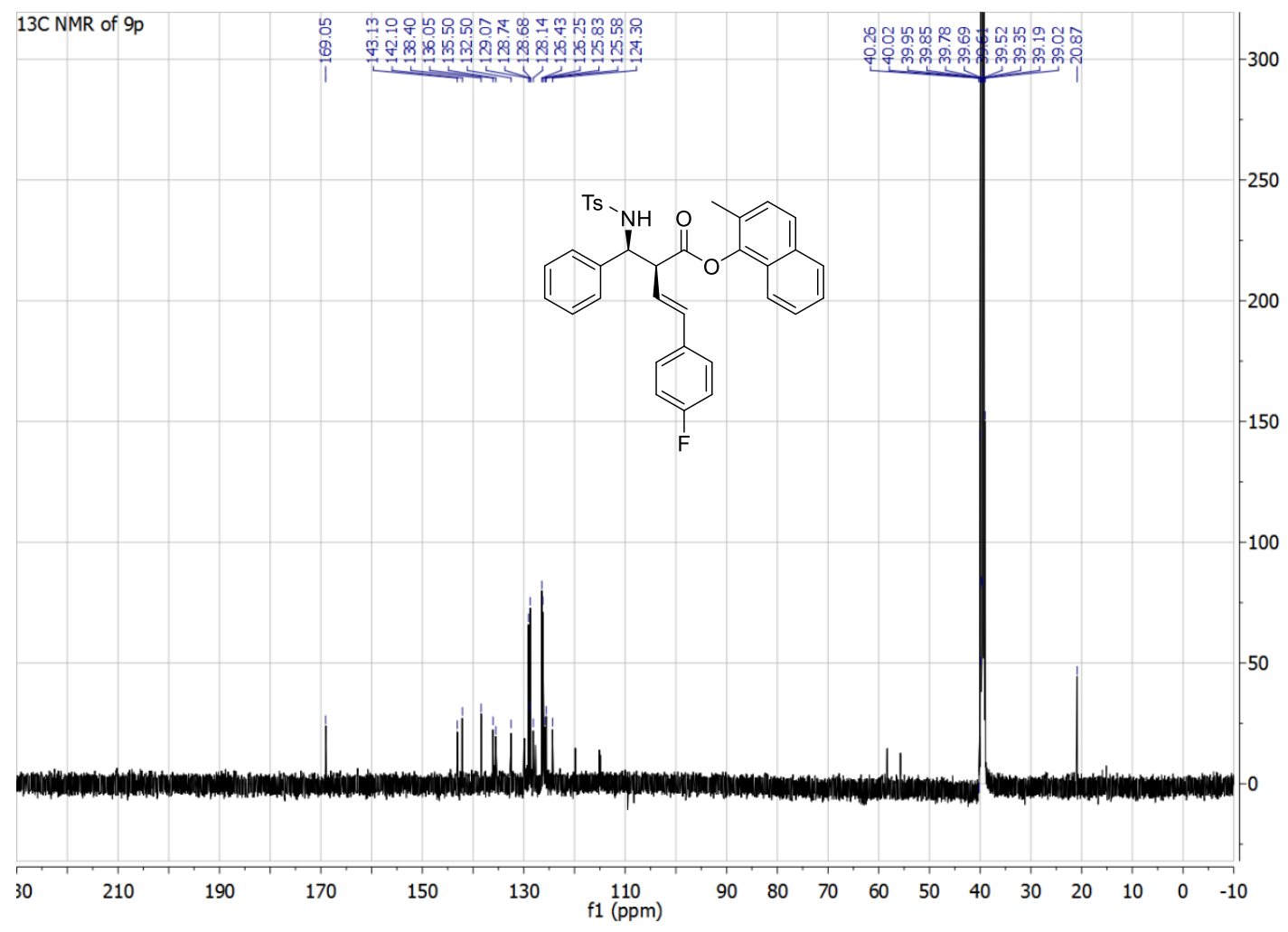

${ }^{13} \mathrm{C}$ NMR of $9 p$ 


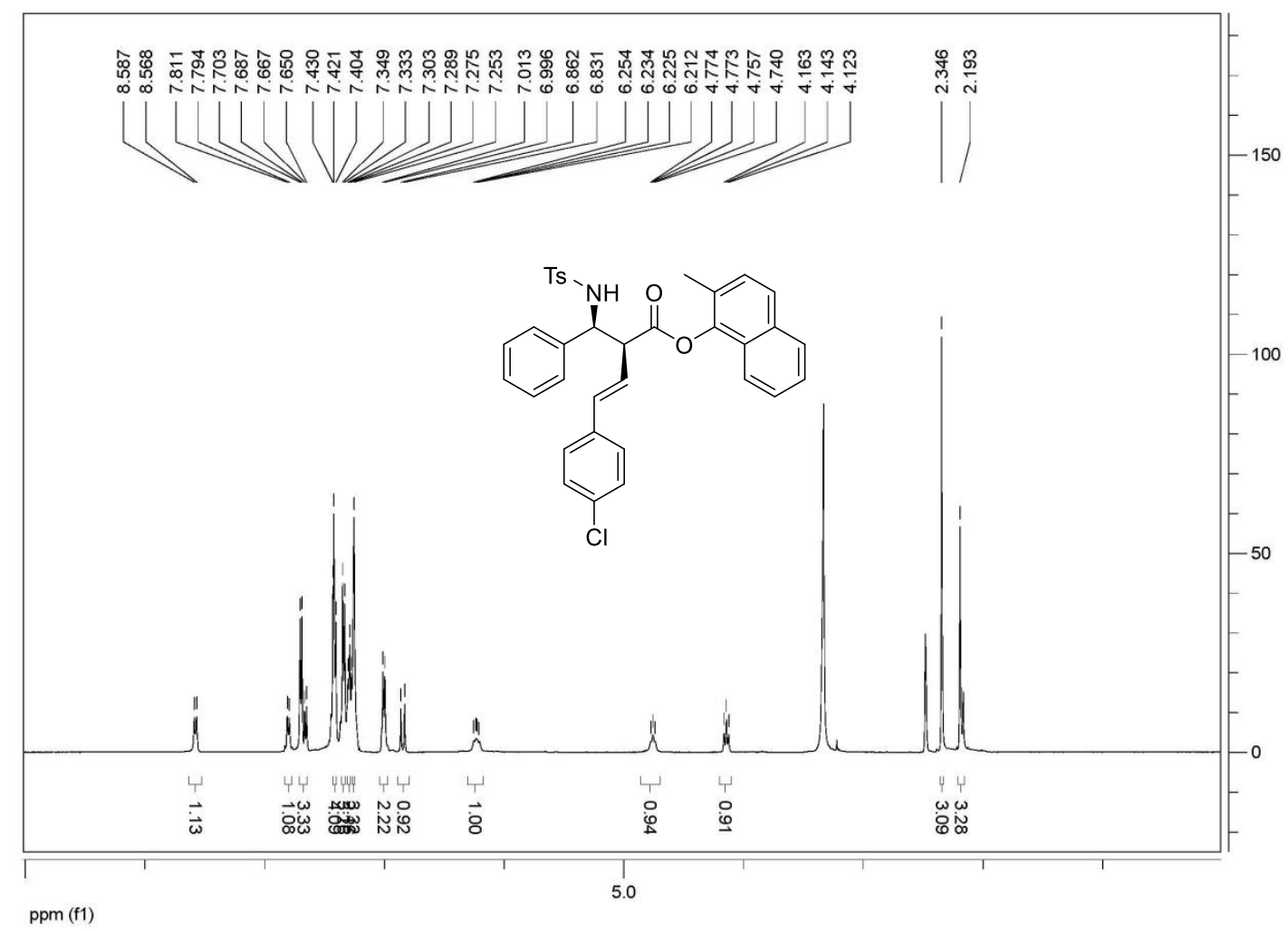

${ }^{1} \mathrm{H}$ NMR of $\mathbf{9 q}$

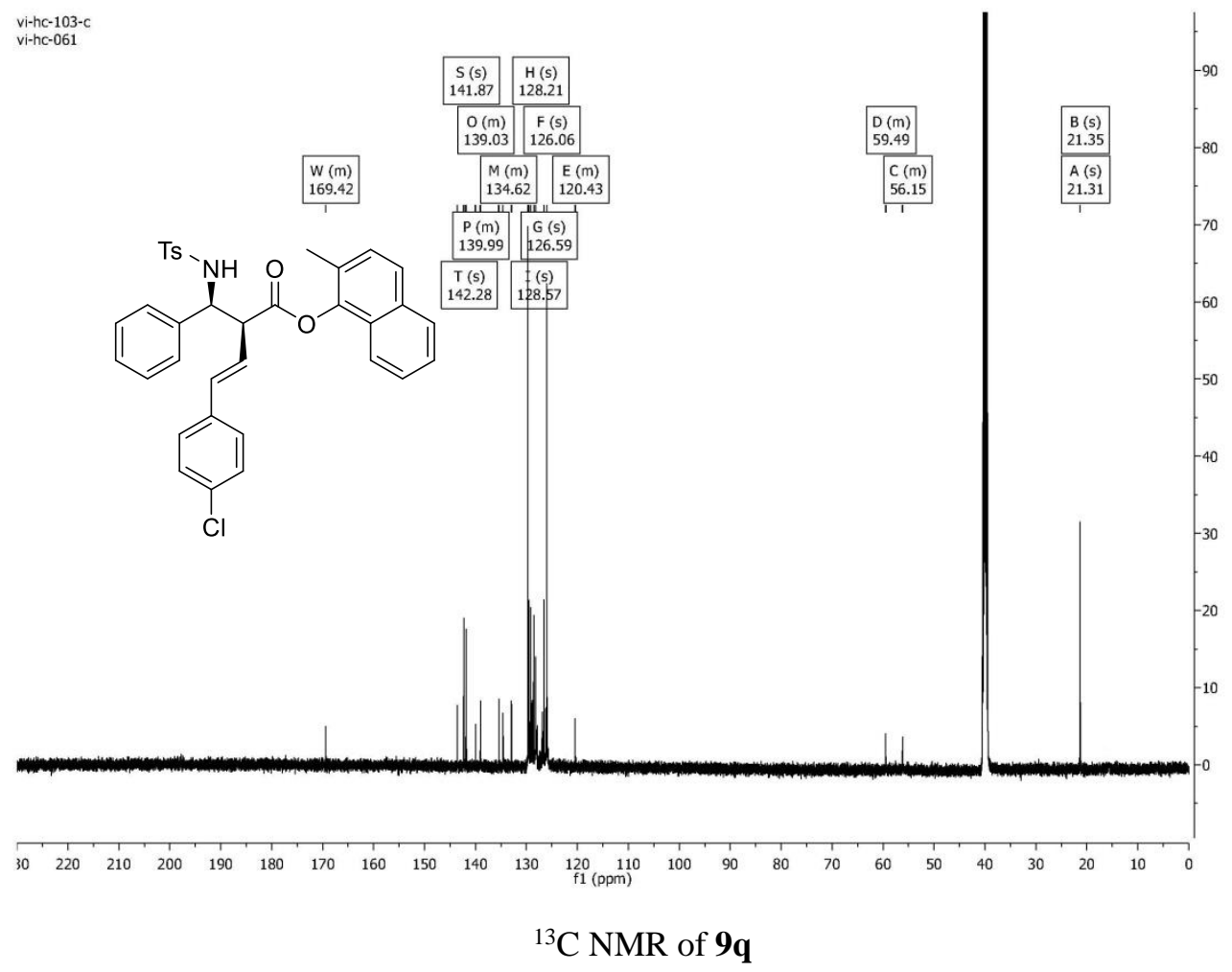




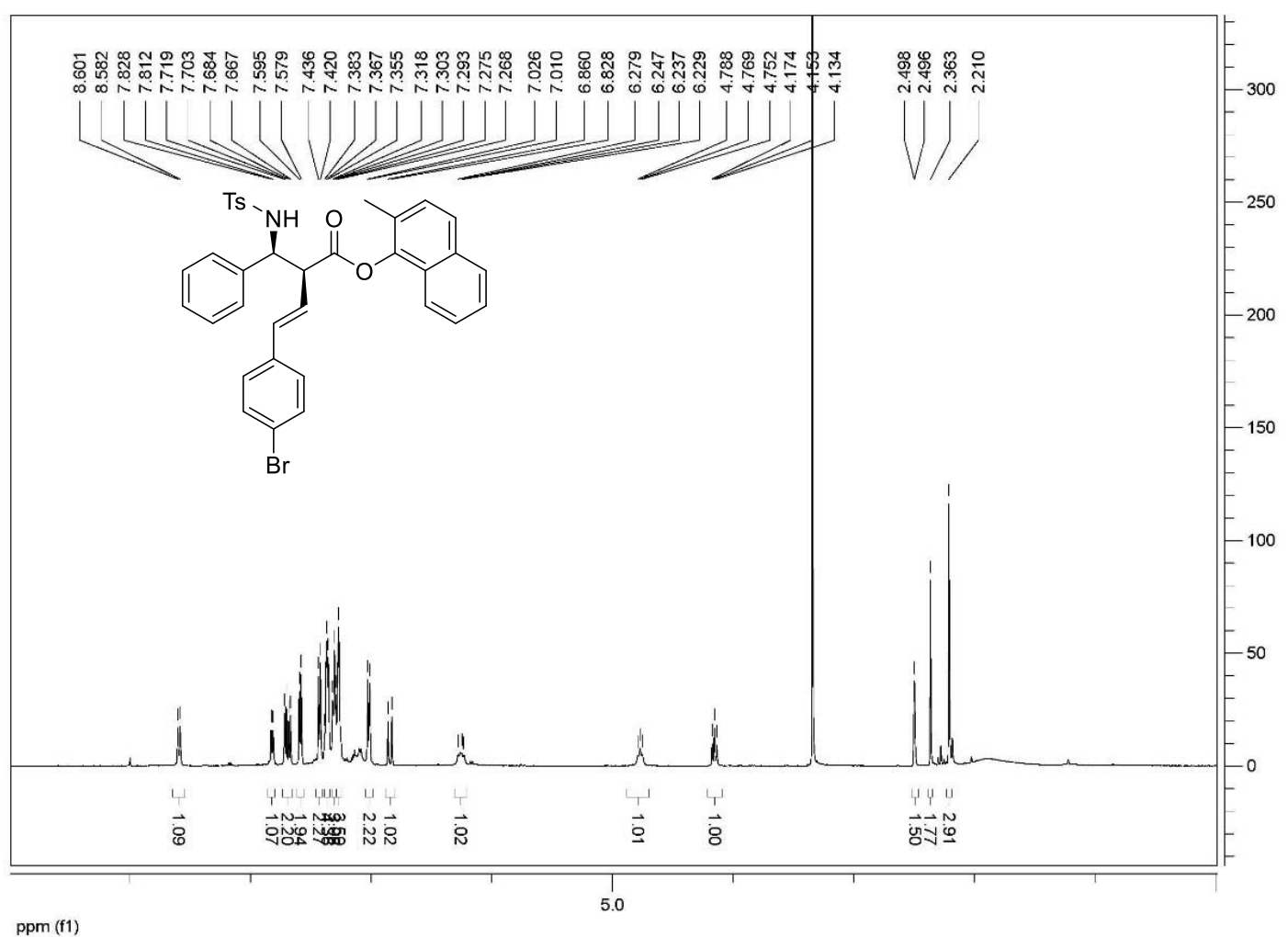

${ }^{1} \mathrm{H}$ NMR of $9 \mathbf{r}$

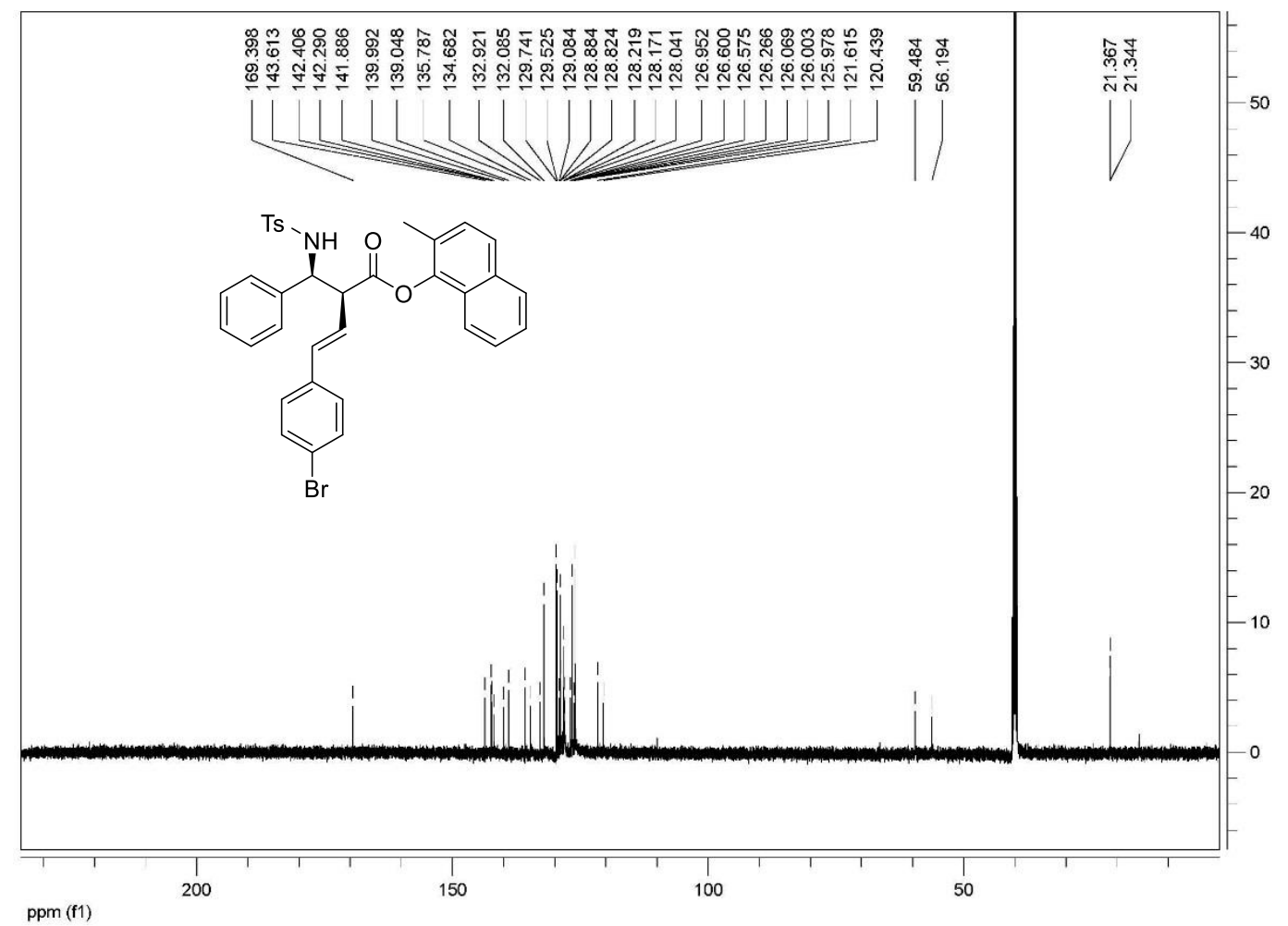

${ }^{13} \mathrm{C}$ NMR of $9 \mathrm{r}$ 


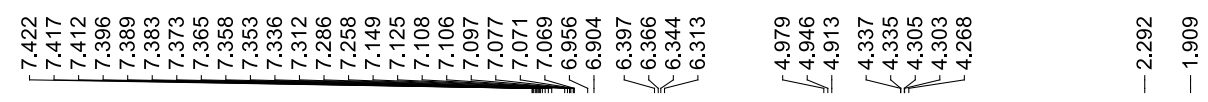

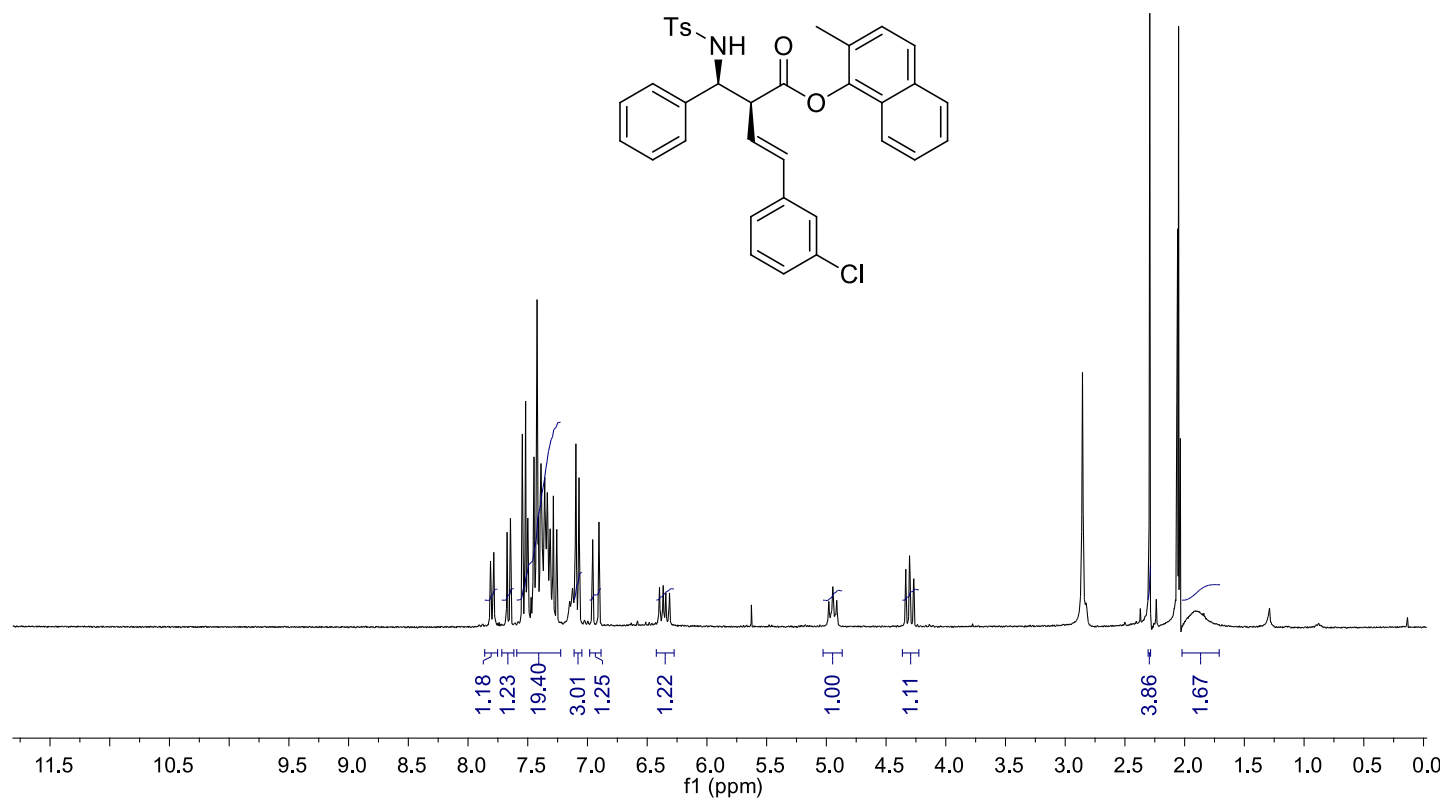

${ }^{1}$ H NMR of 9s

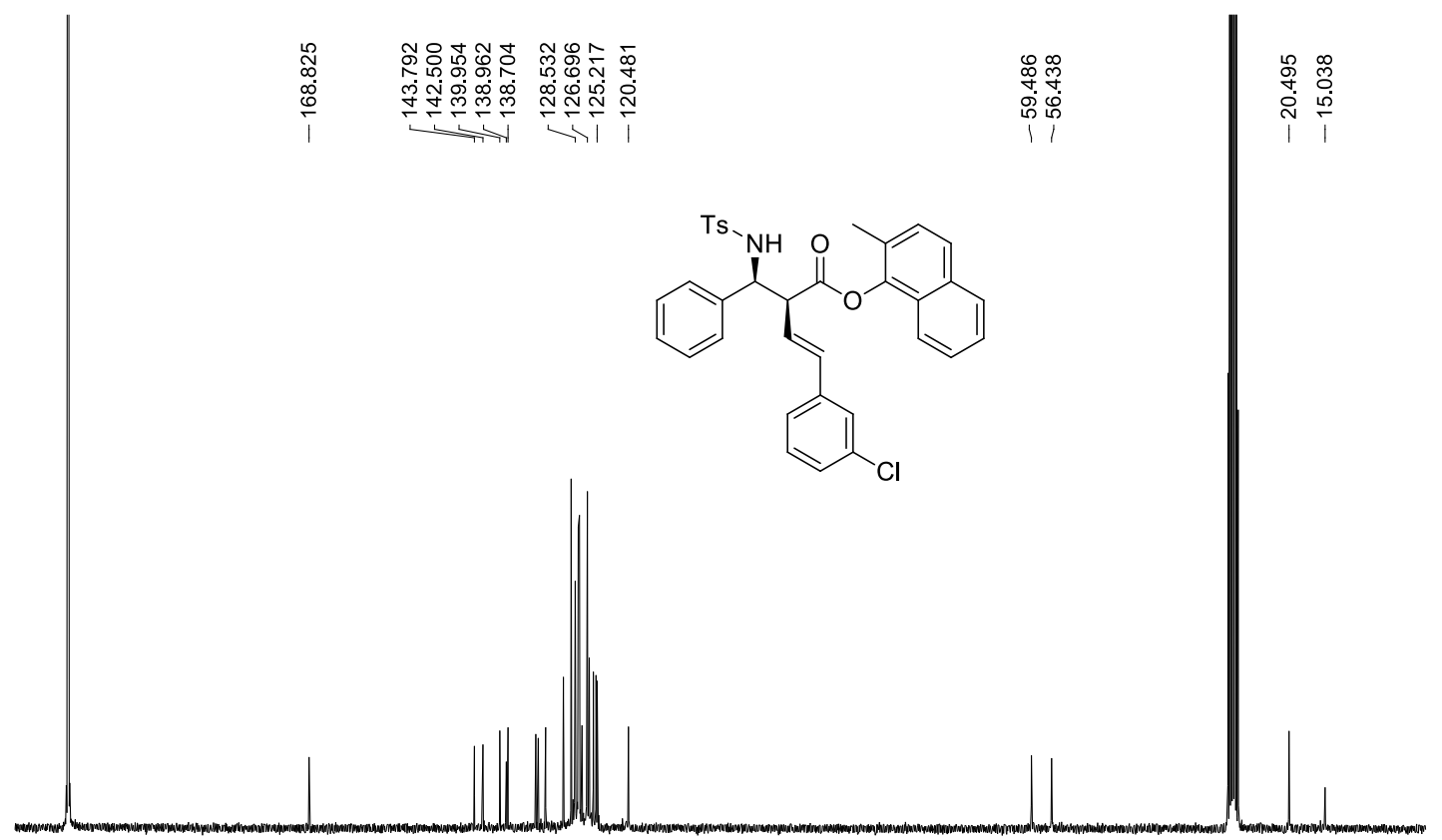

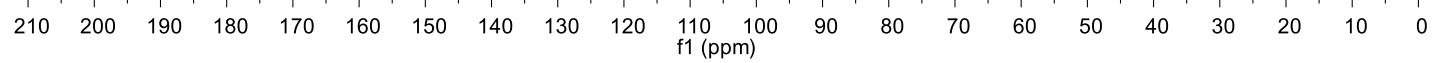

${ }^{13} \mathrm{C}$ NMR of $9 \mathrm{~s}$ 


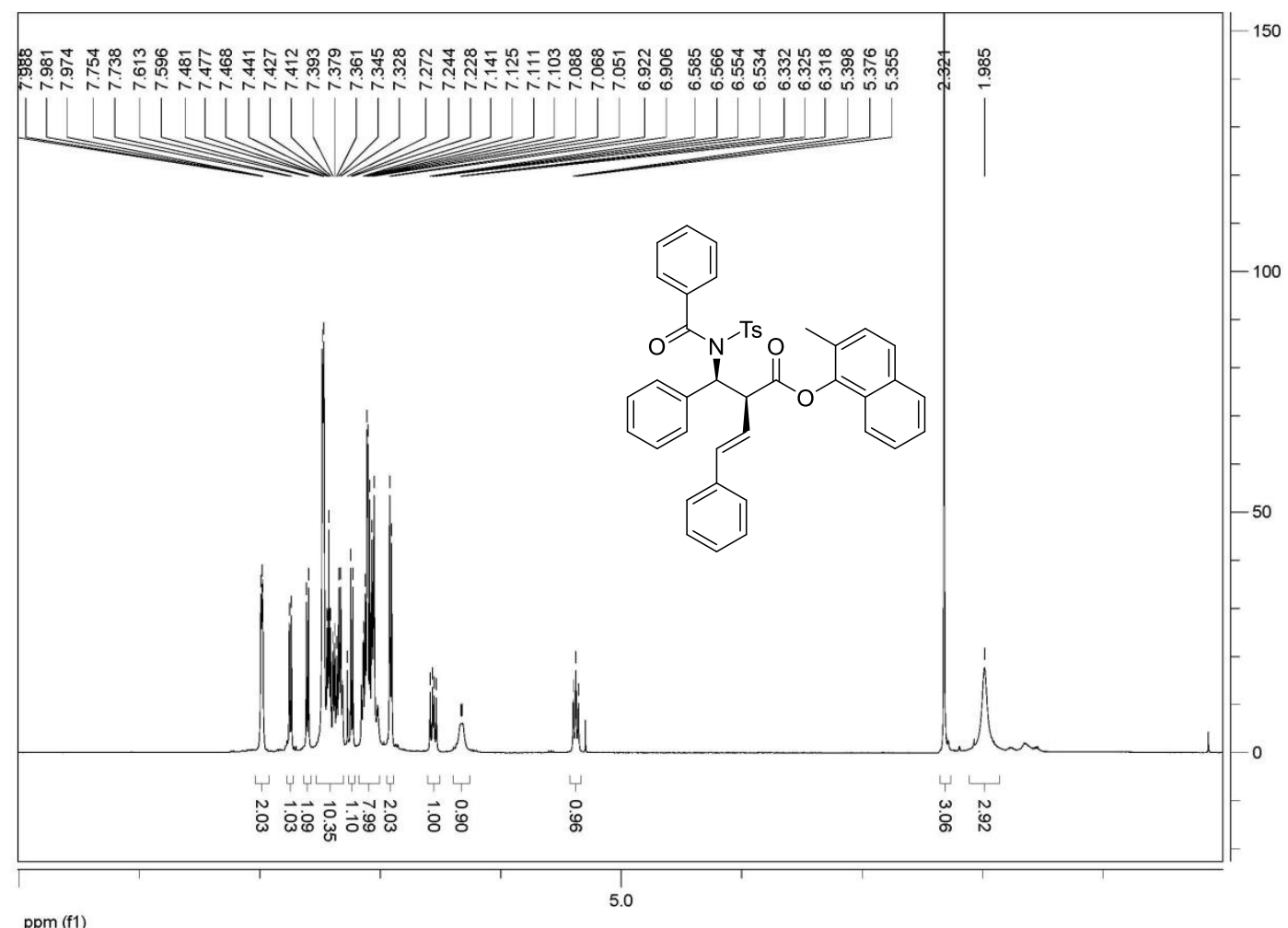

${ }^{1} \mathrm{H}$ NMR of $\mathbf{1 0}$

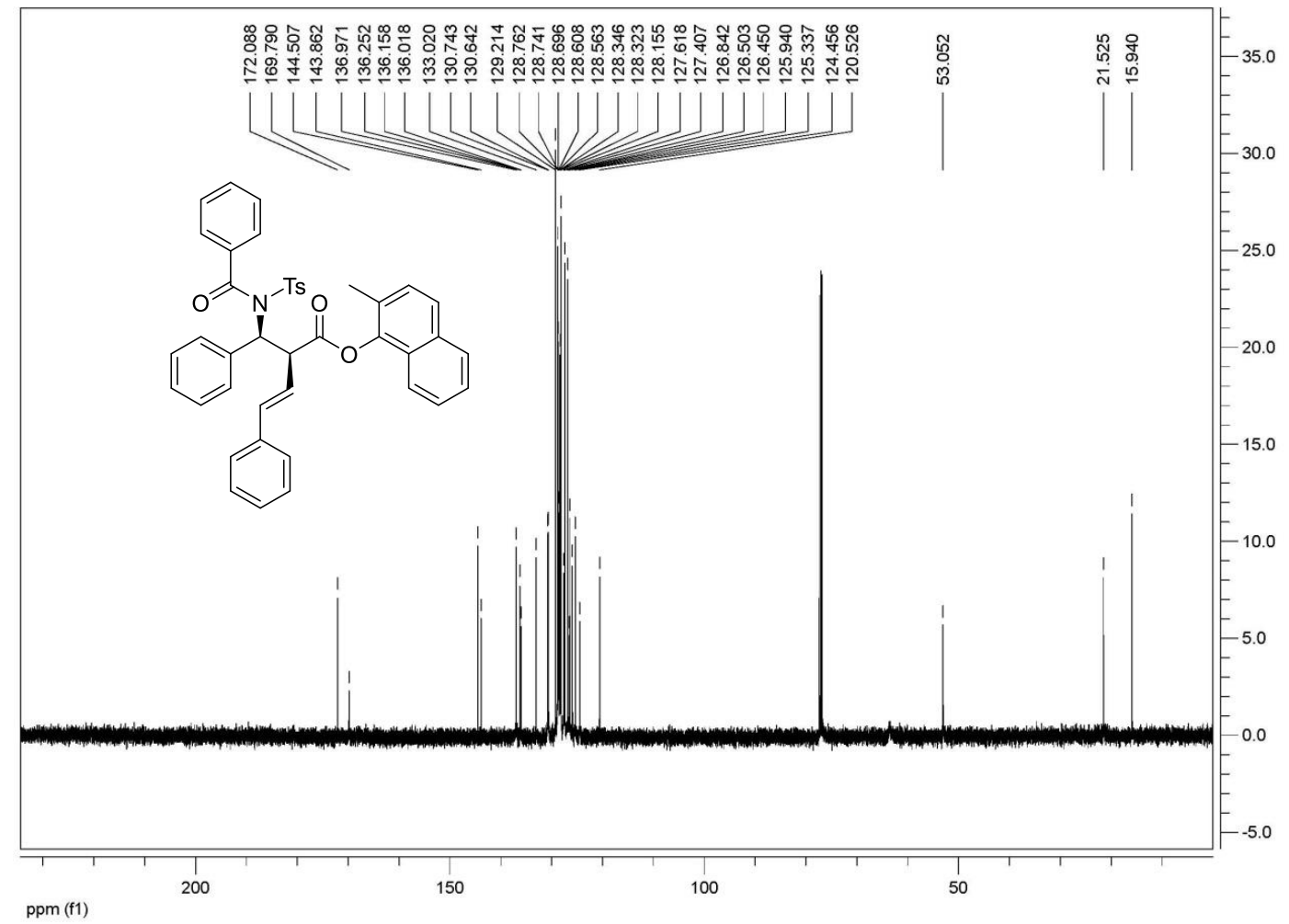

${ }^{13} \mathrm{C}$ NMR of $\mathbf{1 0}$ 


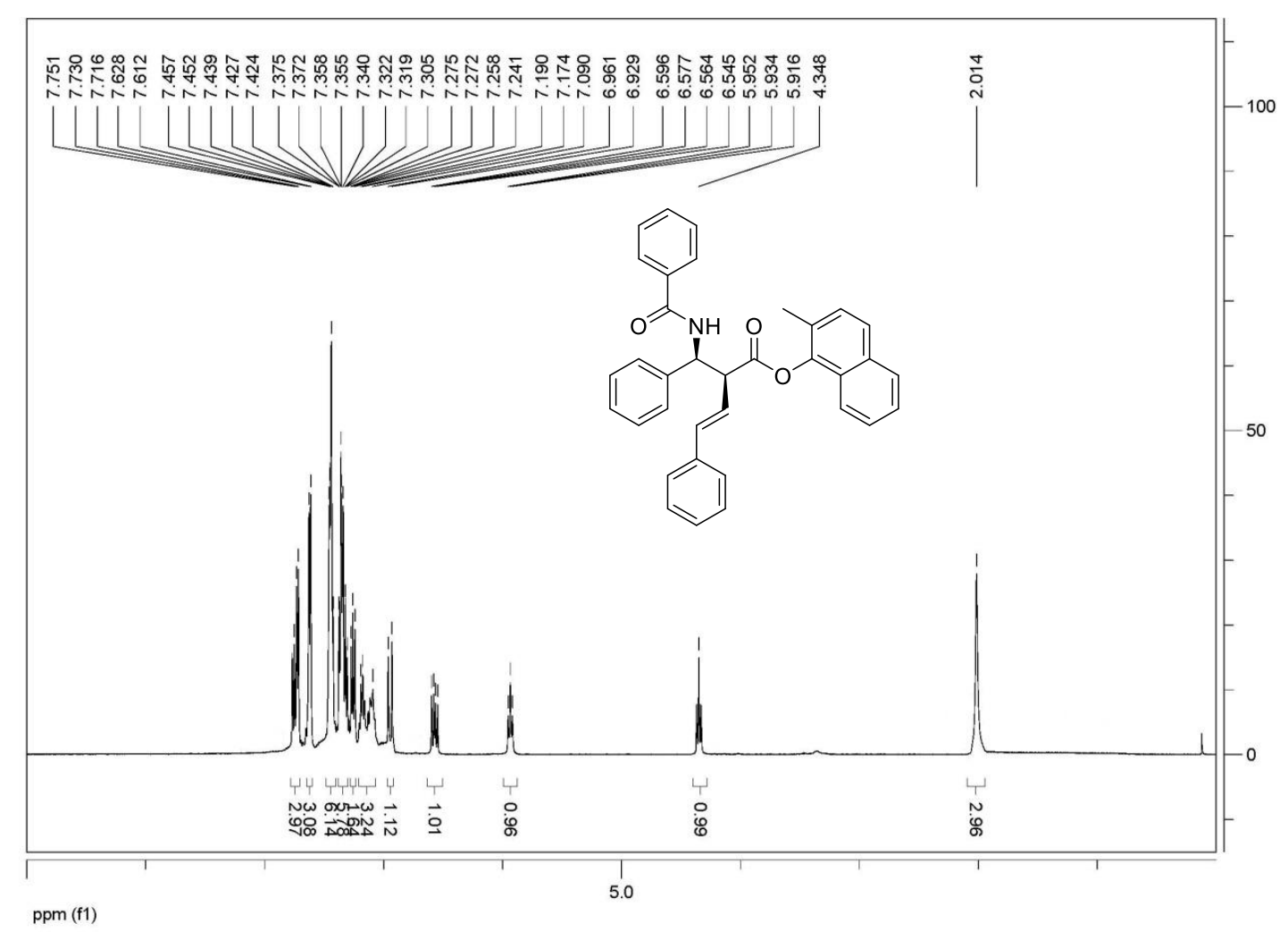

${ }^{1} \mathrm{H}$ NMR of 11

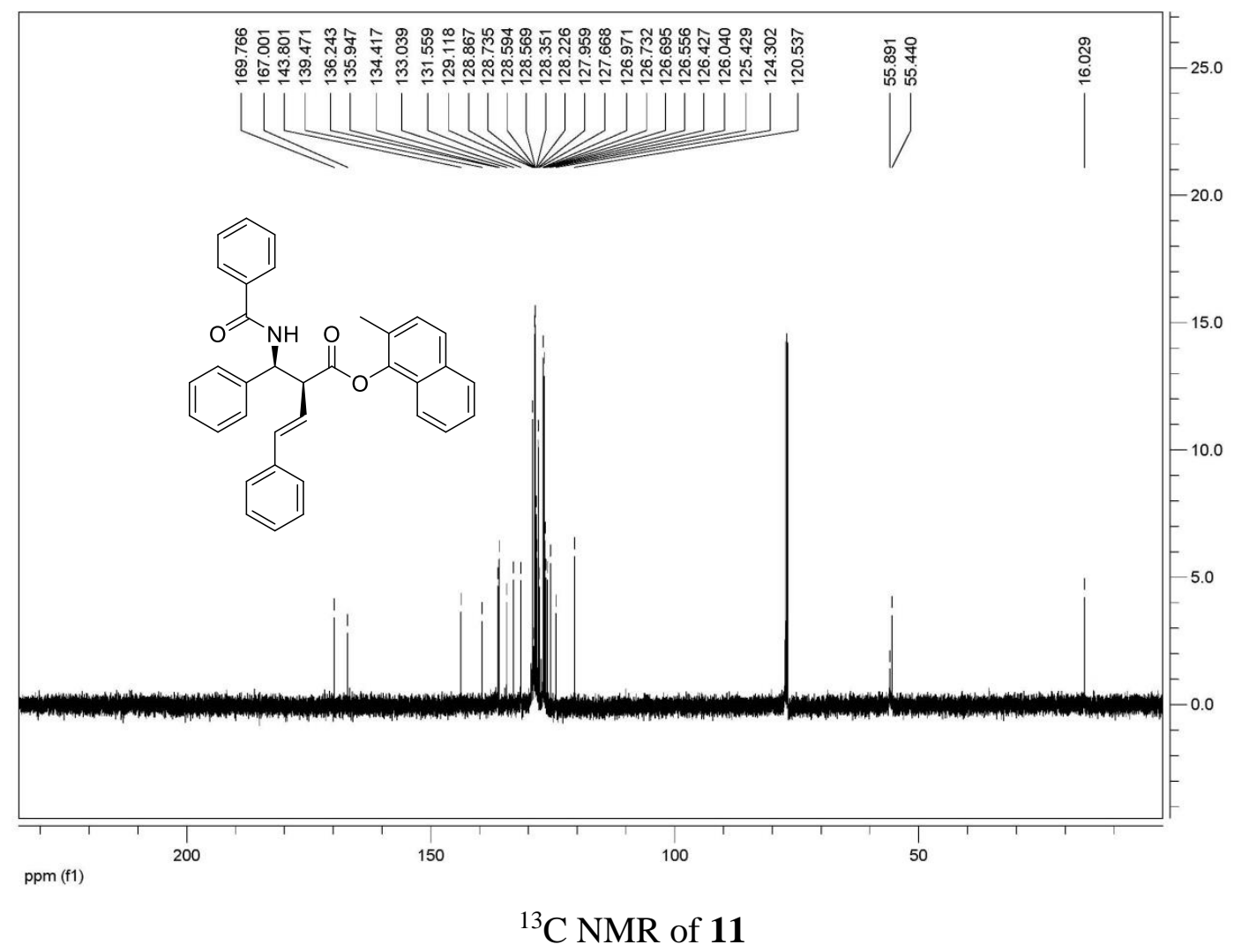




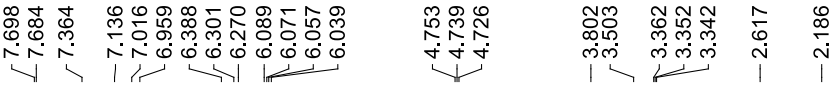

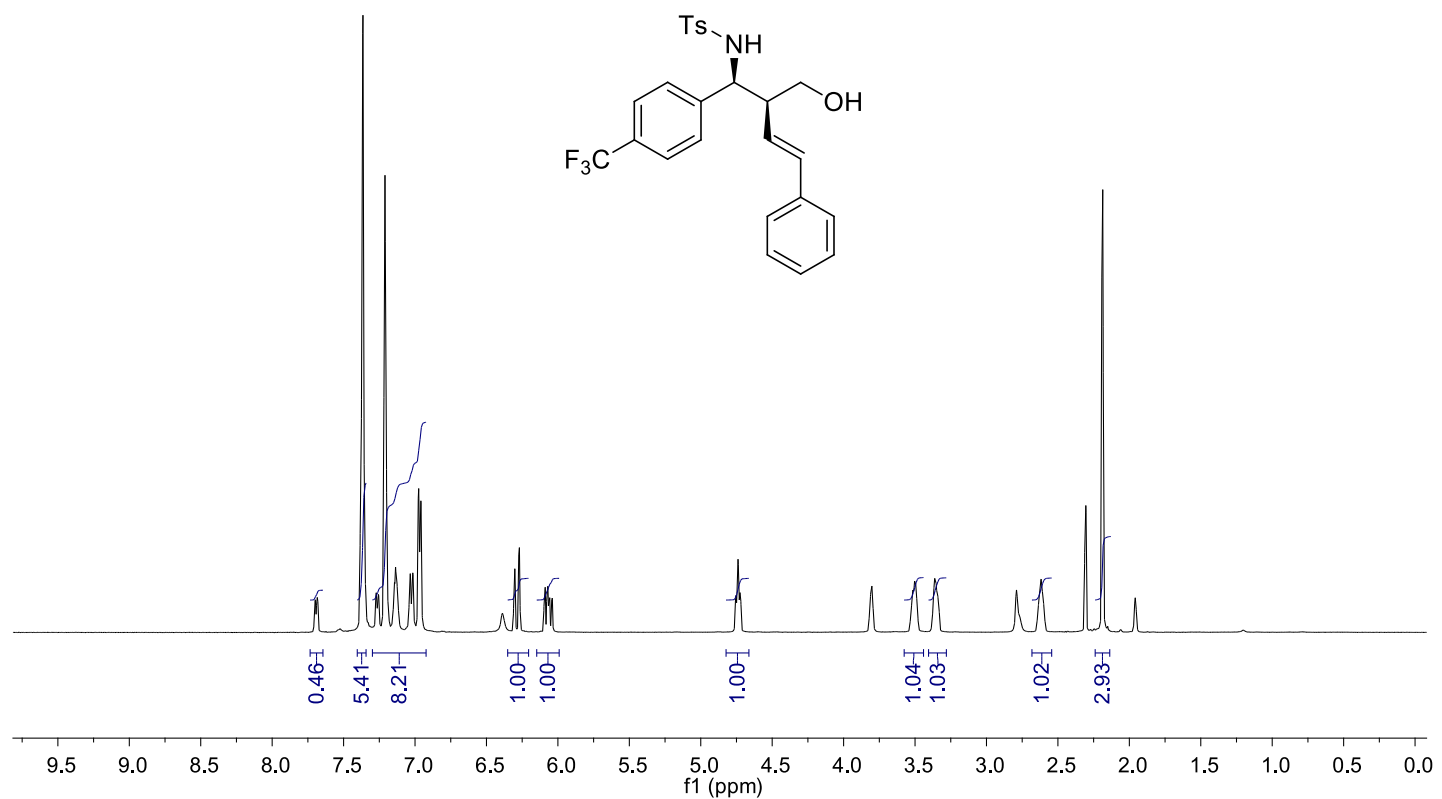

${ }^{1} \mathrm{H}$ NMR of 12

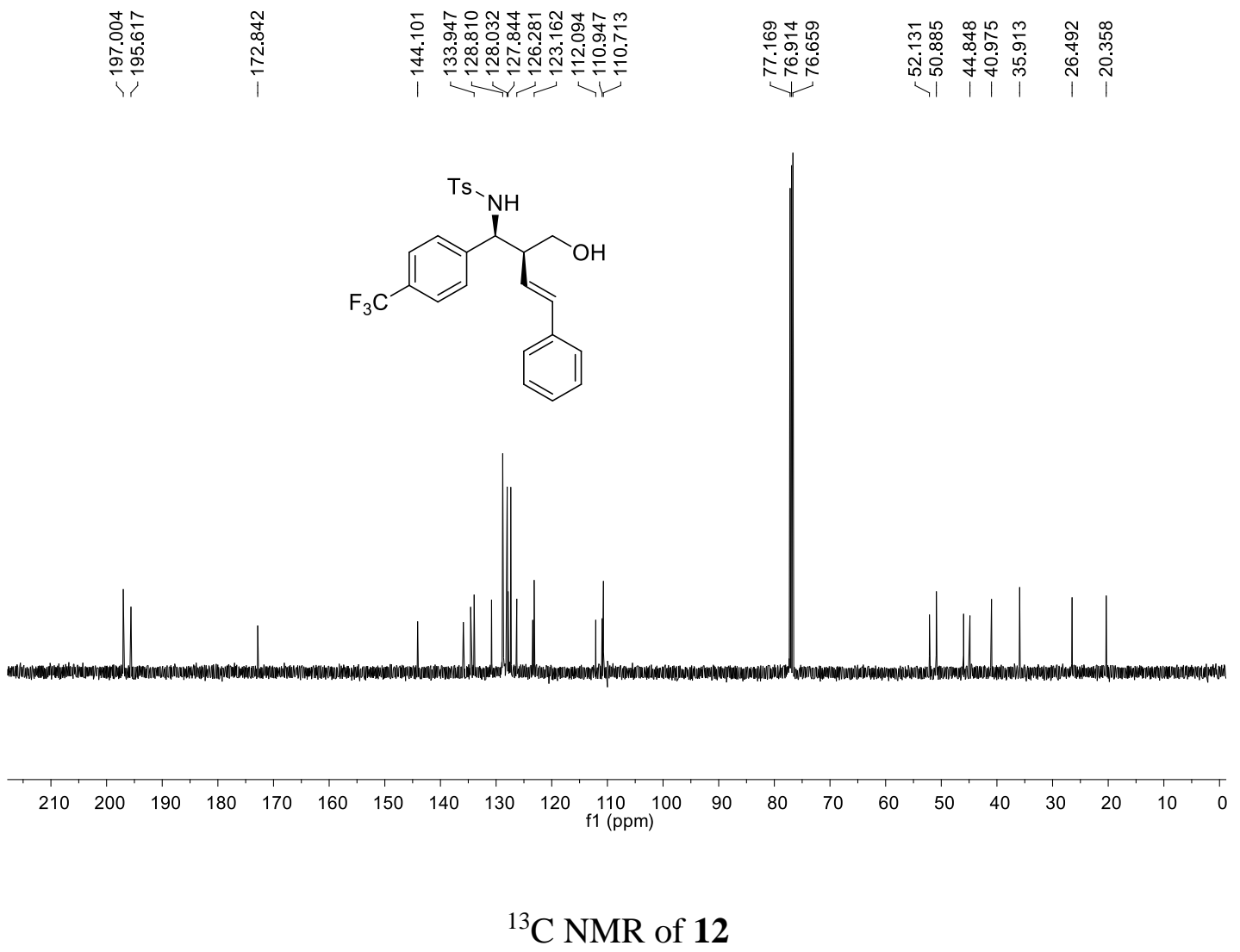



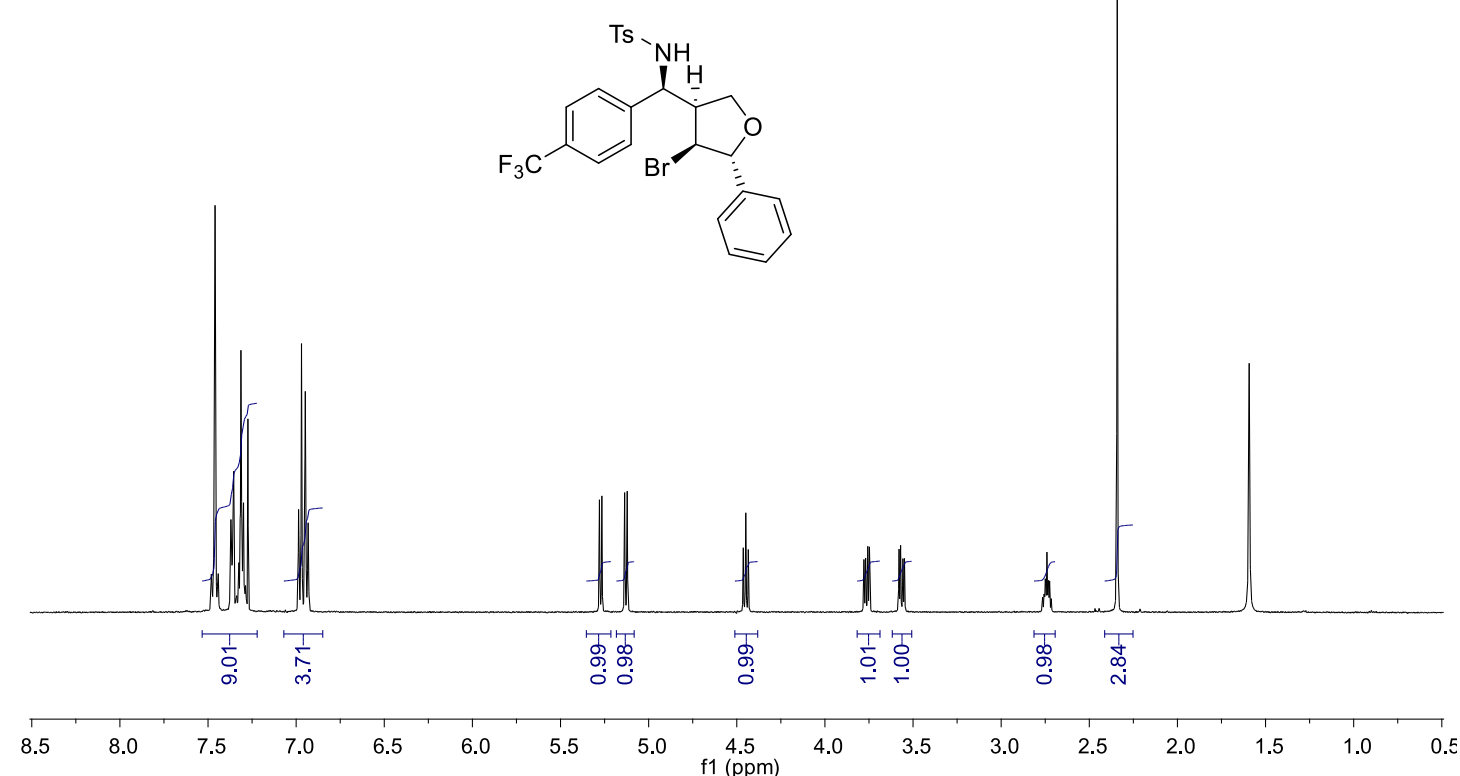

${ }^{1} \mathrm{H}$ NMR of $\mathbf{1 3}$

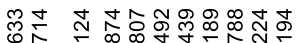

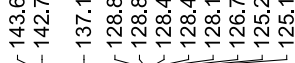

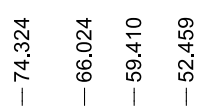

$\stackrel{\stackrel{0}{m}}{\stackrel{i}{i}}$
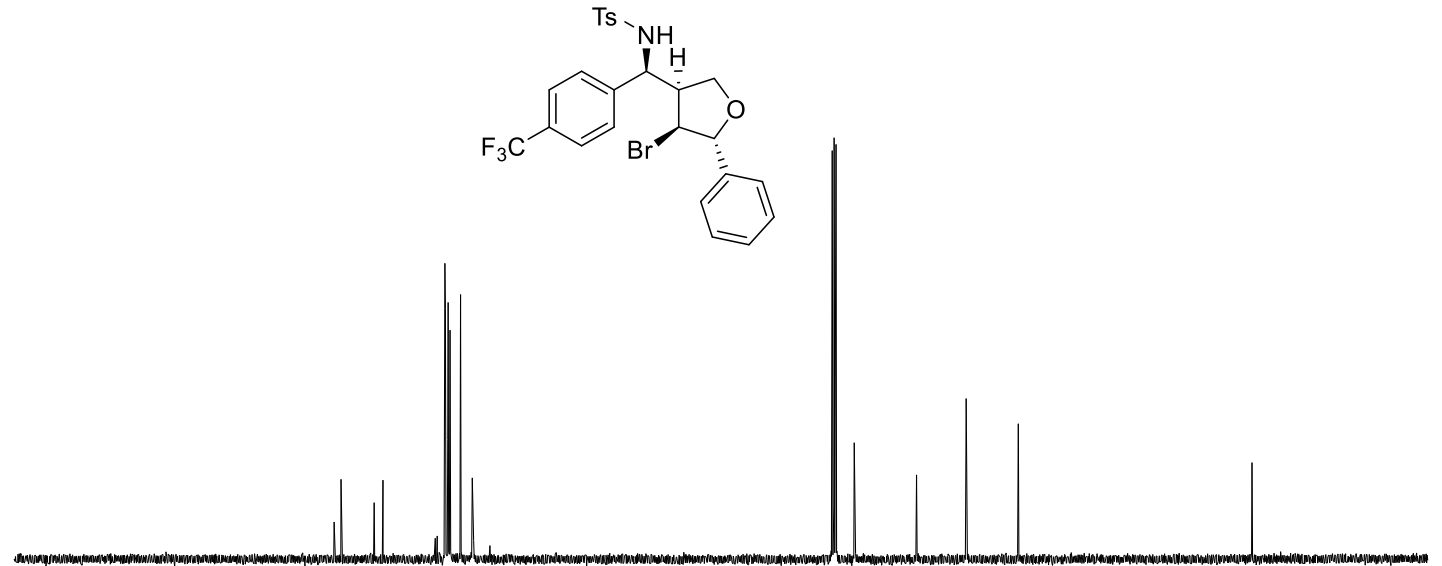

${ }^{13} \mathrm{C}$ NMR of 13 


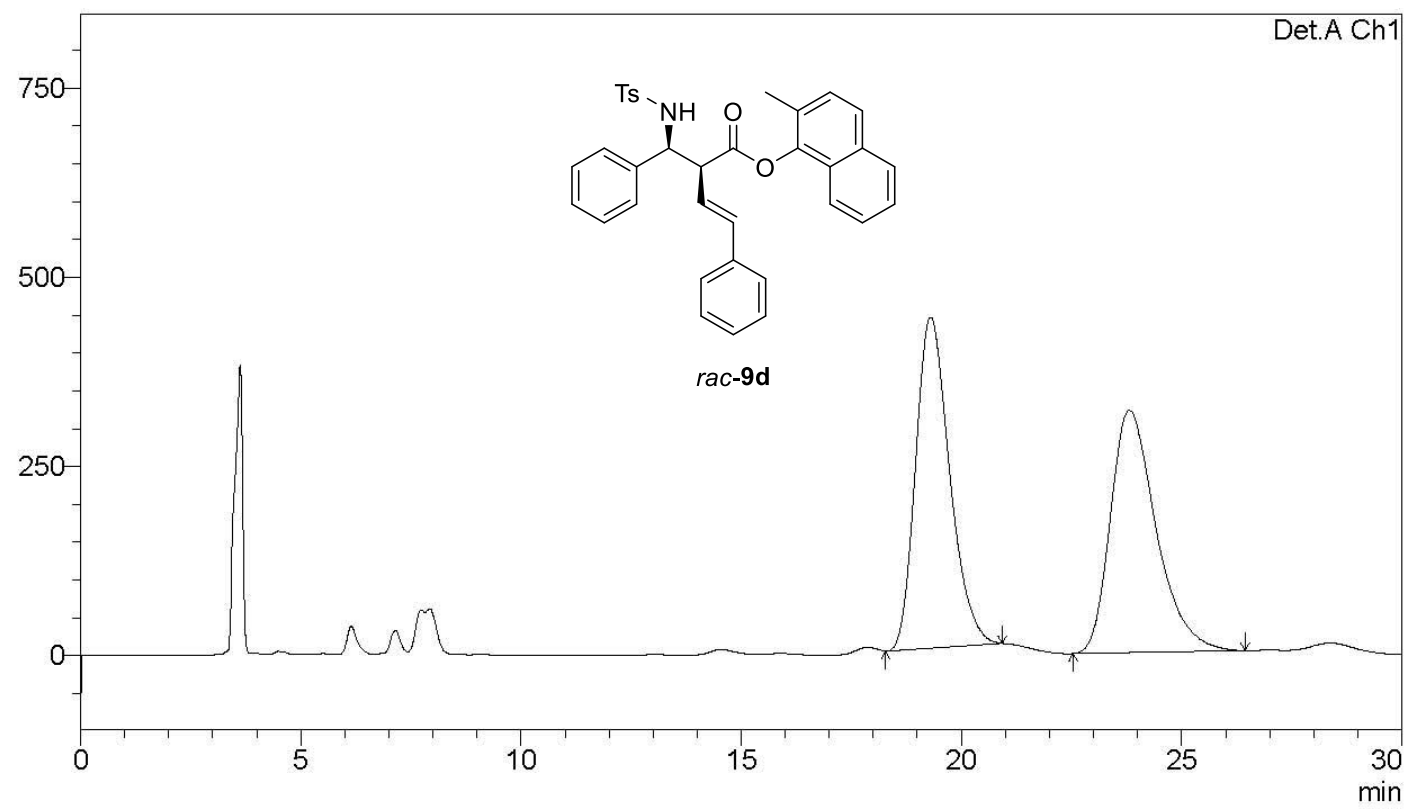

Detector A Ch1 220nm
\begin{tabular}{|r|r|r|r|r|r|}
\hline Peak\# & Ret. Time & Area & Height & \multicolumn{1}{|c|}{ Area \% } & \multicolumn{1}{|c|}{ Height \% } \\
\hline 1 & 19.297 & 23414638 & 437310 & 50.507 & 57.758 \\
\hline 2 & 23.812 & 22944786 & 319829 & 49.493 & 42.242 \\
\hline Total & & 46359424 & 757139 & 100.000 & 100.000 \\
\hline
\end{tabular}

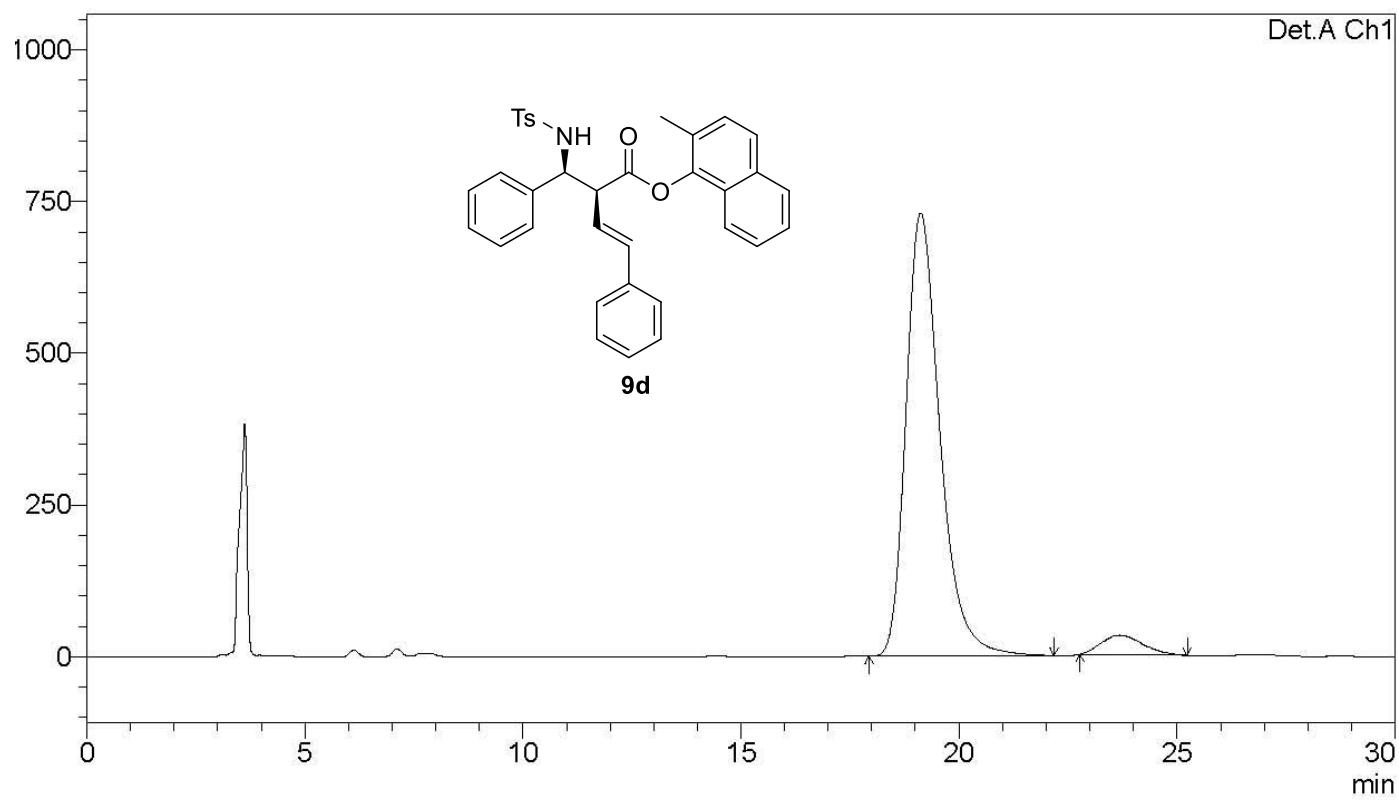

PeakTable

Detector A Chl 220nm
\begin{tabular}{|r|r|r|r|r|r|}
\hline \multicolumn{1}{|c|}{ Peak\# } & Ret. Time & \multicolumn{1}{|c|}{ Area } & Height & Area \% & Height \% \\
\hline 1 & 19.117 & 39341548 & 729533 & 94.897 & 95.777 \\
\hline 2 & 23.679 & 2115764 & 32167 & 5.103 & 4.223 \\
\hline Total & & 41457312 & 761700 & 100.000 & 100.000 \\
\hline
\end{tabular}


==== Shimadzu LCsolution LC Data Image ====

$<$ Chromatogram>
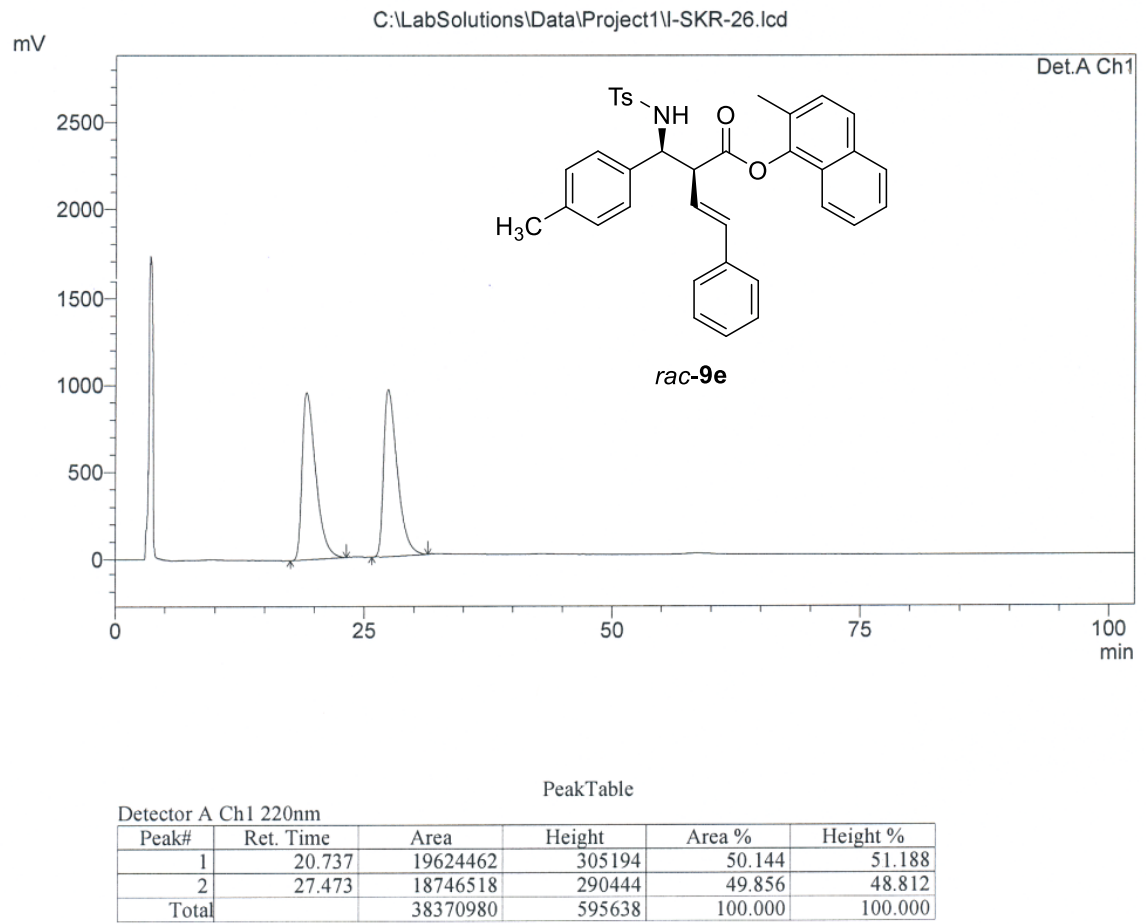

$====$ Shimadzu LCsolution LC Data Image ====

$<$ Chromatogram $>$

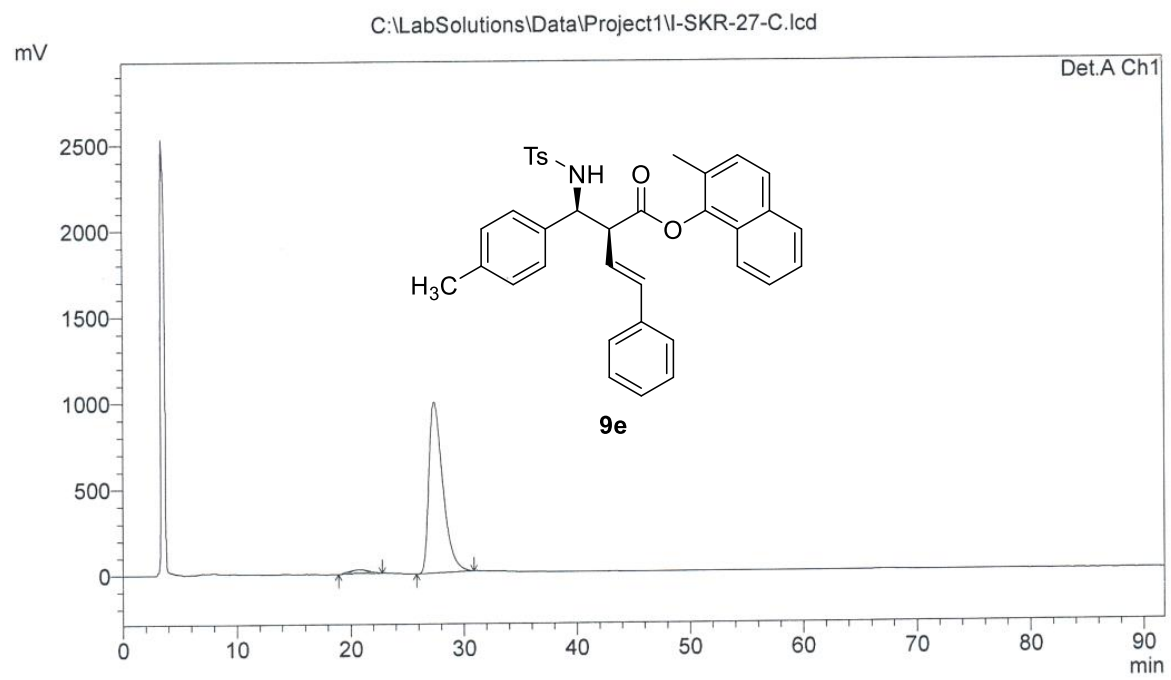

\begin{tabular}{|c|c|c|c|c|c|}
\hline \multirow{2}{*}{\multicolumn{6}{|c|}{ Detector A Ch1 $220 \mathrm{~nm}$}} \\
\hline & & & & & \\
\hline Peak\# & Ret. Time & Area & Height & Area \% & Height \% \\
\hline 1 & 20.841 & 48133 & 1028 & 7.056 & 6.103 \\
\hline 2 & 27.377 & 85728083 & 993408 & 93.944 & 93.897 \\
\hline Total & & 85776216 & 994436 & 100.000 & 100.000 \\
\hline
\end{tabular}




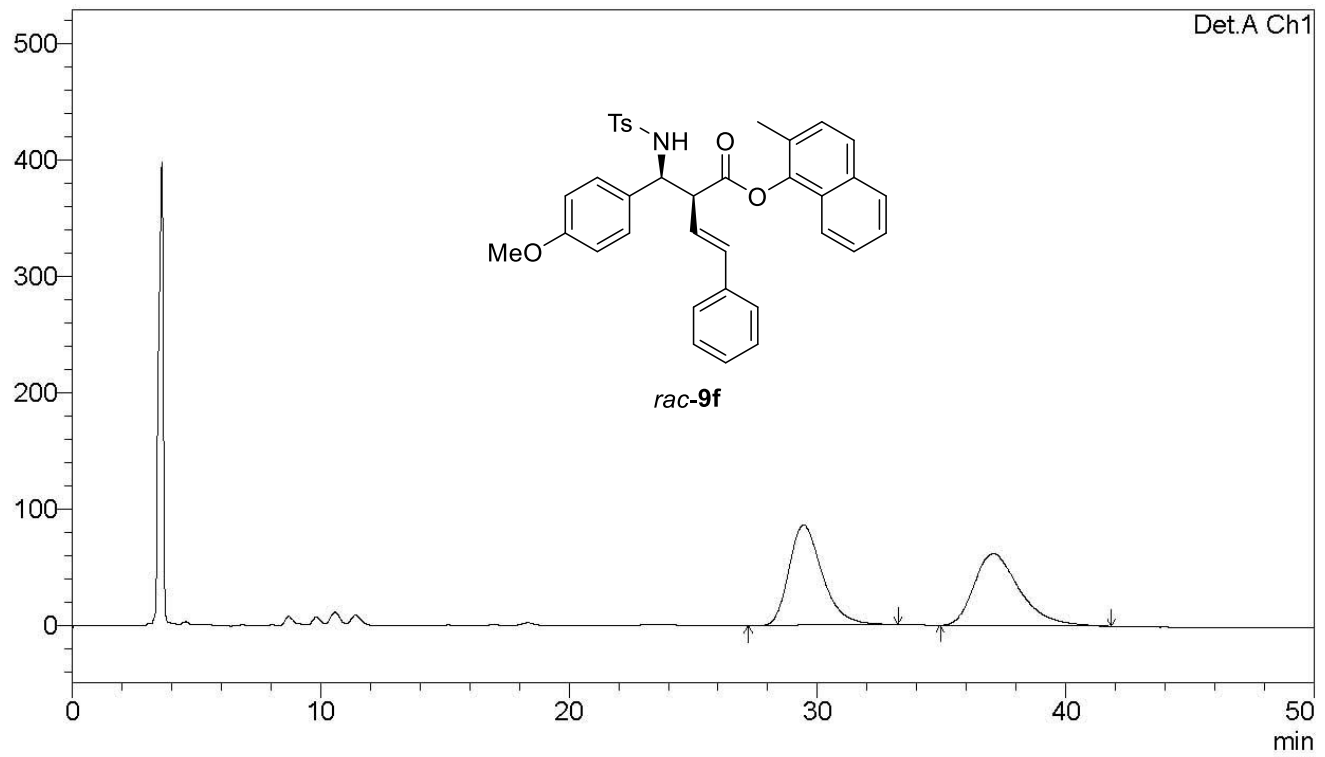

Detector A Ch1 220nm
\begin{tabular}{r|r|r|r|r|r|}
\hline \multicolumn{1}{|c|}{ Peak\# } & Ret. Time & Area & Height & Area \% & \multicolumn{1}{|c|}{ Height \% } \\
\hline 1 & 29.426 & 7930034 & 86000 & 50.341 & 58.266 \\
\hline 2 & 37.068 & 7822494 & 61599 & 49.659 & 41.734 \\
\hline Total & & 15752528 & 147599 & 100.000 & 100.000 \\
\hline
\end{tabular}

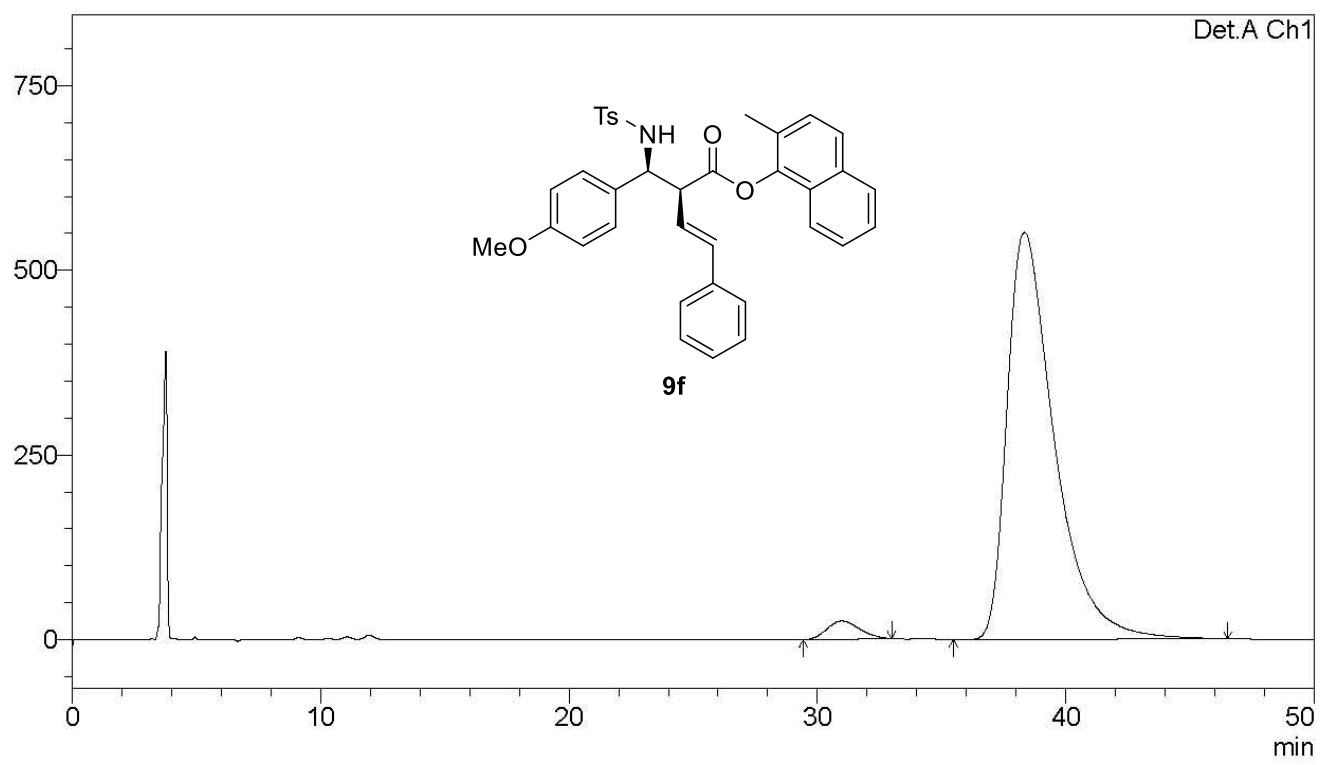

PeakTable

Detector A Ch1 220nm
\begin{tabular}{|r|r|r|r|r|r|}
\hline Peak\# & Ret. Time & Area & Height & Area \% & Height \% \\
\hline 1 & 30.980 & 2249041 & \multicolumn{1}{c|}{24712} & 2.999 & 4.294 \\
\hline 2 & 38.321 & 72732411 & 550781 & 97.001 & 95.706 \\
\hline Total & & 74981452 & 575493 & 100.000 & 100.000 \\
\hline
\end{tabular}




\section{Area \% Report}

Page 1 of 1

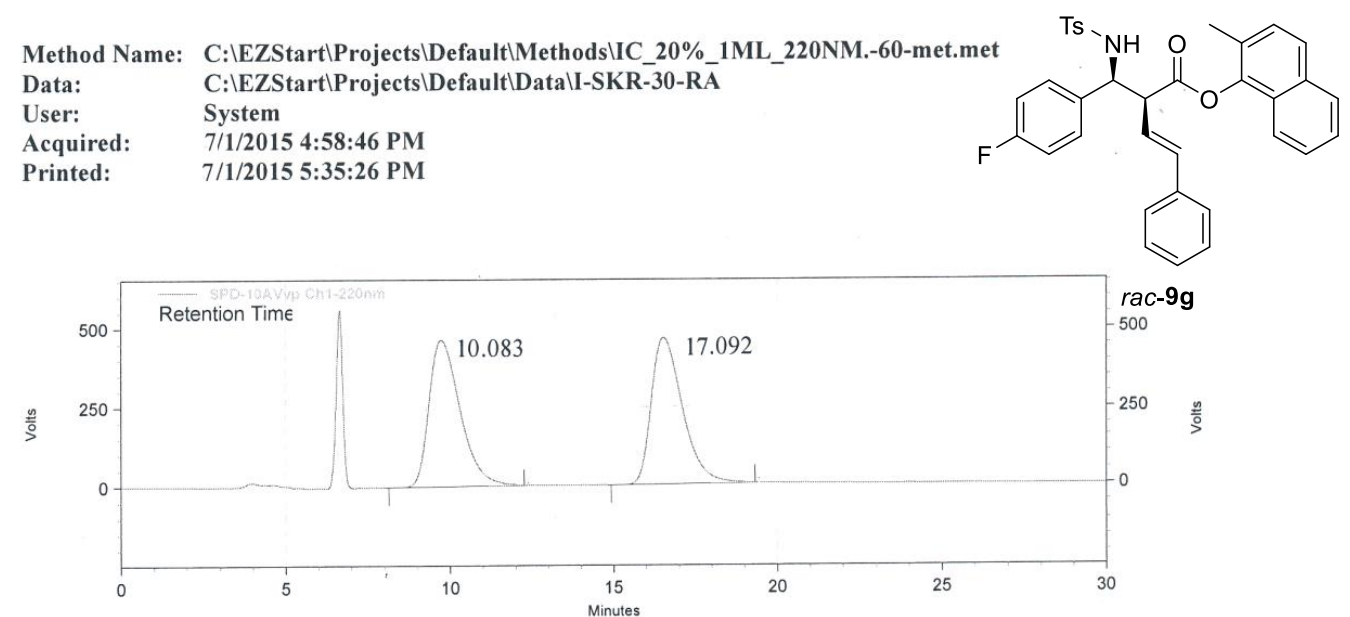

SPD-10AVvp

Ch1-220nm

Results

\begin{tabular}{rrrrrr}
$\begin{array}{c}\text { Results } \\
\text { Retention Time }\end{array}$ & Area & Area \% & Height & Height \% \\
\hline 10.083 & 2292235 & 50.41 & 47744 & 59.69 \\
17.092 & 2255187 & 49.59 & 32244 & 40.31 \\
\hline Totals & 4547422 & 100.00 & 79988 & 100.00 \\
\hline
\end{tabular}

Page 1 of 1

\section{Area \% Report}

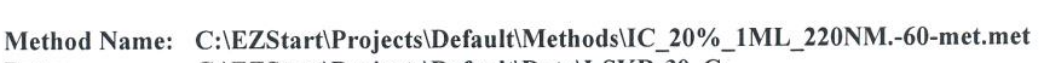

Data: C:IEZStart $\backslash$ Projects $\backslash$ Default $\backslash$ Data $\backslash I-S K R-30-C$

User: System

Acquired: $\quad$ 4/1/2015 4:58:46 PM

Printed: $\quad$ 4/1/2015 5:35:26 PM
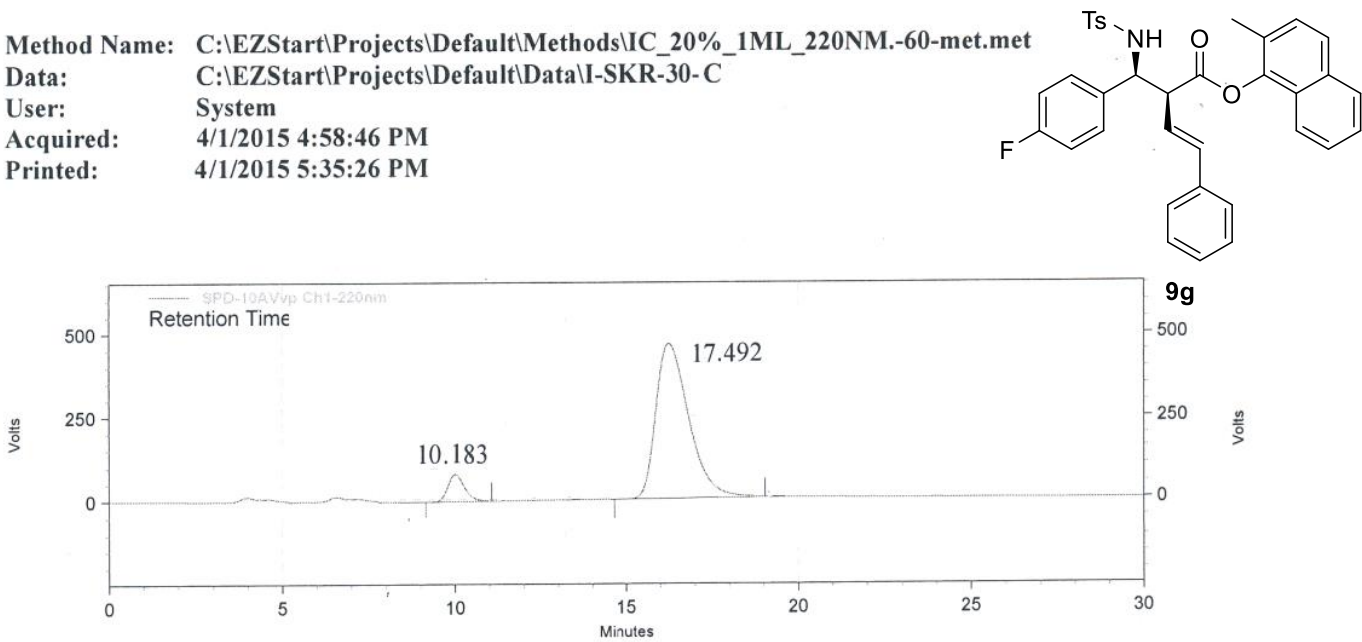

SPD-10AVvp

Ch1-220nm

Results

\begin{tabular}{rrrrrr|}
$\begin{array}{r}\text { Retention Time } \\
\text { Area }\end{array}$ & Area \% & Height & Height \% \\
\hline 10.183 & 3073928 & 7.14 & 46223 & 10.12 \\
17.492 & 39991674 & 92.86 & 410398 & 89.88 \\
\multicolumn{2}{|c|}{ Totals } & 43065602 & 100.00 & 456621 & 100.00 \\
\hline
\end{tabular}




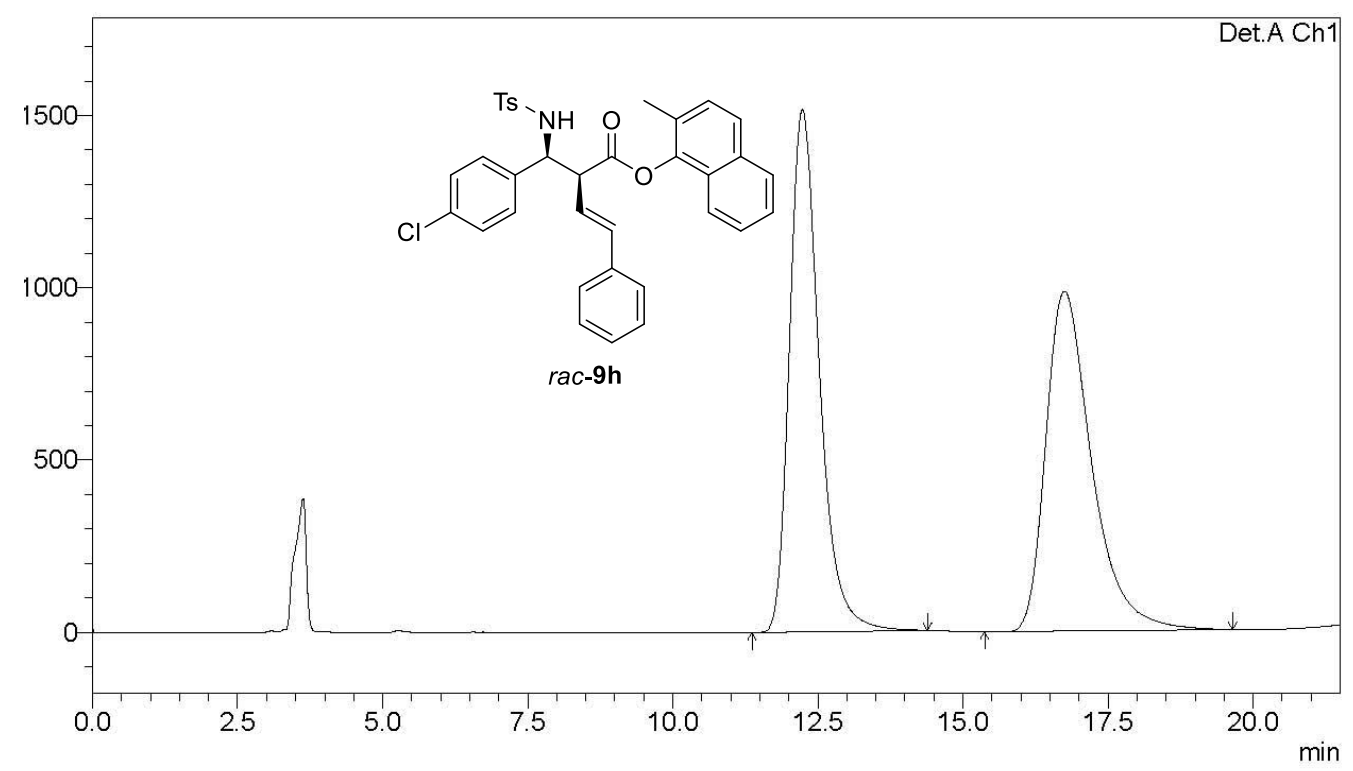

PeakTable

Detector A Ch1 220nm
\begin{tabular}{|r|r|r|r|r|r|}
\hline Peak\# & Ret. Time & \multicolumn{1}{|c|}{ Area } & \multicolumn{1}{c|}{ Height } & Area \% & Height \% \\
\hline 1 & 12.230 & 54126434 & 1516527 & 50.010 & 60.602 \\
\hline 2 & 16.744 & 54104707 & 985893 & 49.990 & 39.398 \\
\hline Total & & 108231141 & 2502420 & 100.000 & 100.000 \\
\hline
\end{tabular}

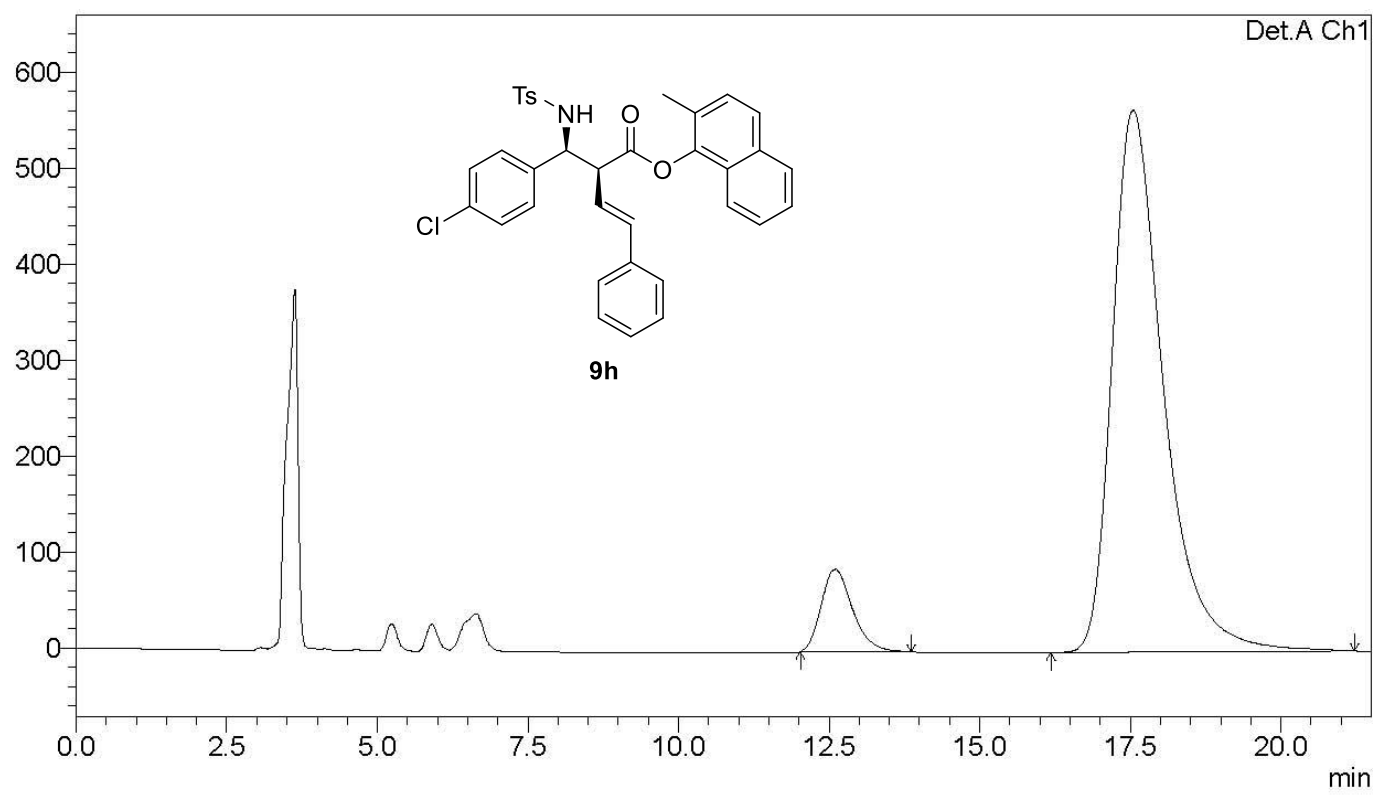

PeakTable

Detector A Chl $220 \mathrm{~nm}$
\begin{tabular}{|r|r|r|r|r|r|}
\hline Peak\# & Ret. Time & Area & Height & Area $\%$ & Height $\%$ \\
\hline 1 & 12.595 & 3088555 & 85895 & 8.492 & 13.202 \\
\hline 2 & 17.542 & 33280335 & 564712 & 91.508 & 86.798 \\
\hline Total & & 36368890 & 650608 & 100.000 & 100.000 \\
\hline
\end{tabular}




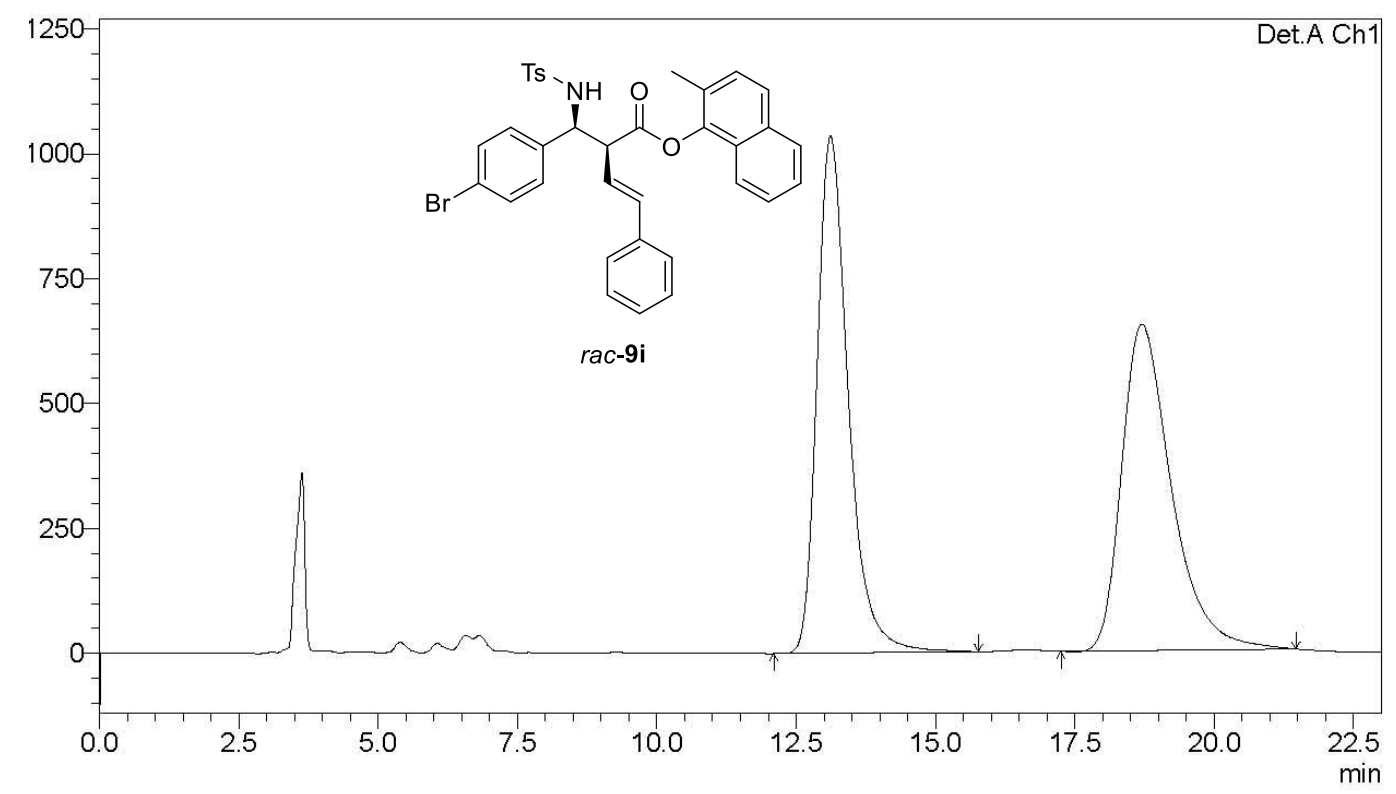

PeakTable

Detector A Ch1 220nm

\begin{tabular}{|r|r|r|r|r|r|}
\hline Peak\# & Ret. Time & \multicolumn{1}{|c|}{ Area } & \multicolumn{1}{|c|}{ Height } & Area \% & Height \% \\
\hline 1 & 13.112 & 40182503 & 1034893 & 49.840 & 61.323 \\
\hline 2 & 18.697 & 40440128 & 652710 & 50.160 & 38.677 \\
\hline Total & & 80622631 & 1687603 & 100.000 & 100.000 \\
\hline
\end{tabular}

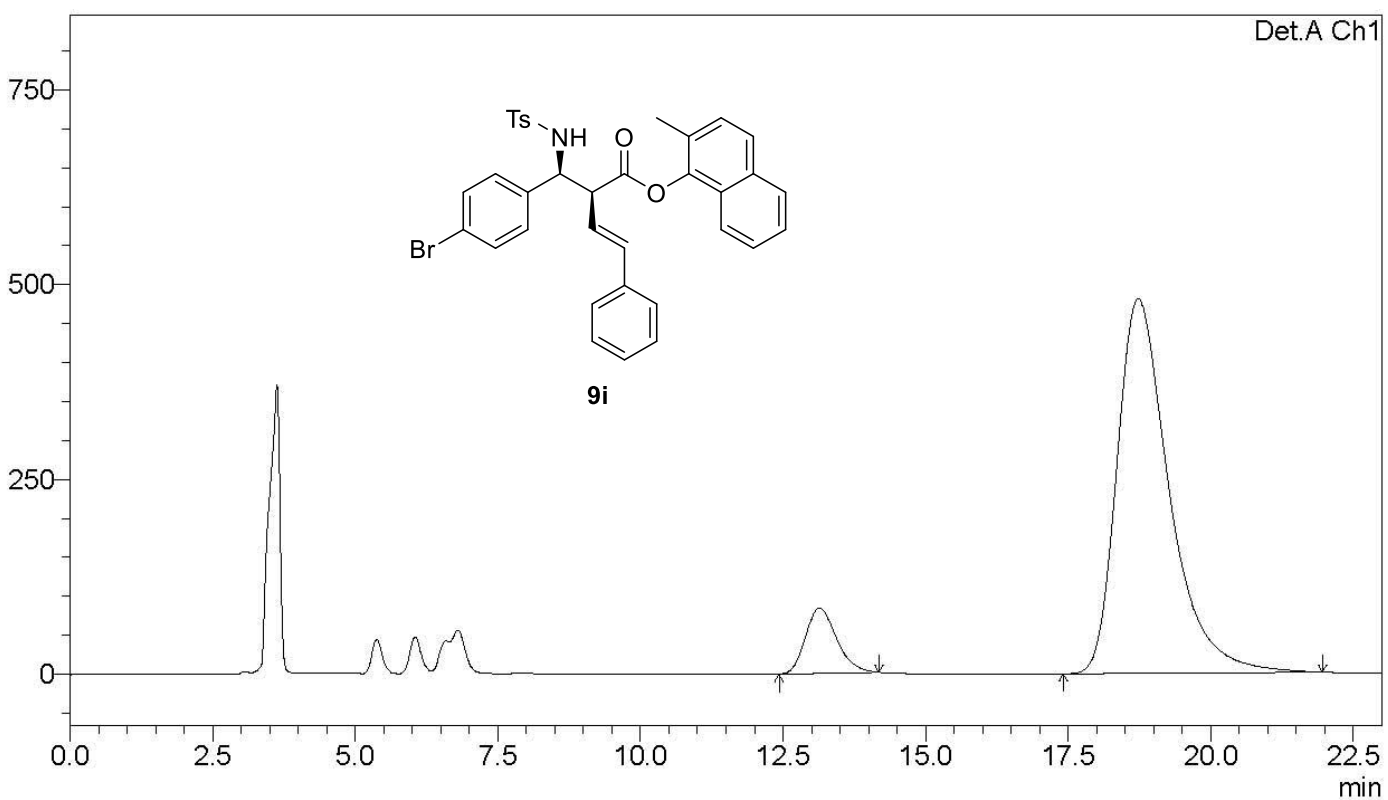

Detector A Ch1 220nm
\begin{tabular}{|r|r|r|r|r|r|}
\hline Peak\# & Ret. Time & Area & Height & Area \% & Height \% \\
\hline 1 & 13.136 & 3151157 & 83419 & 9.386 & 14.800 \\
\hline 2 & 18.724 & 30422869 & 480228 & 90.614 & 85.200 \\
\hline Total & & 33574026 & 563646 & 100.000 & 100.000 \\
\hline
\end{tabular}




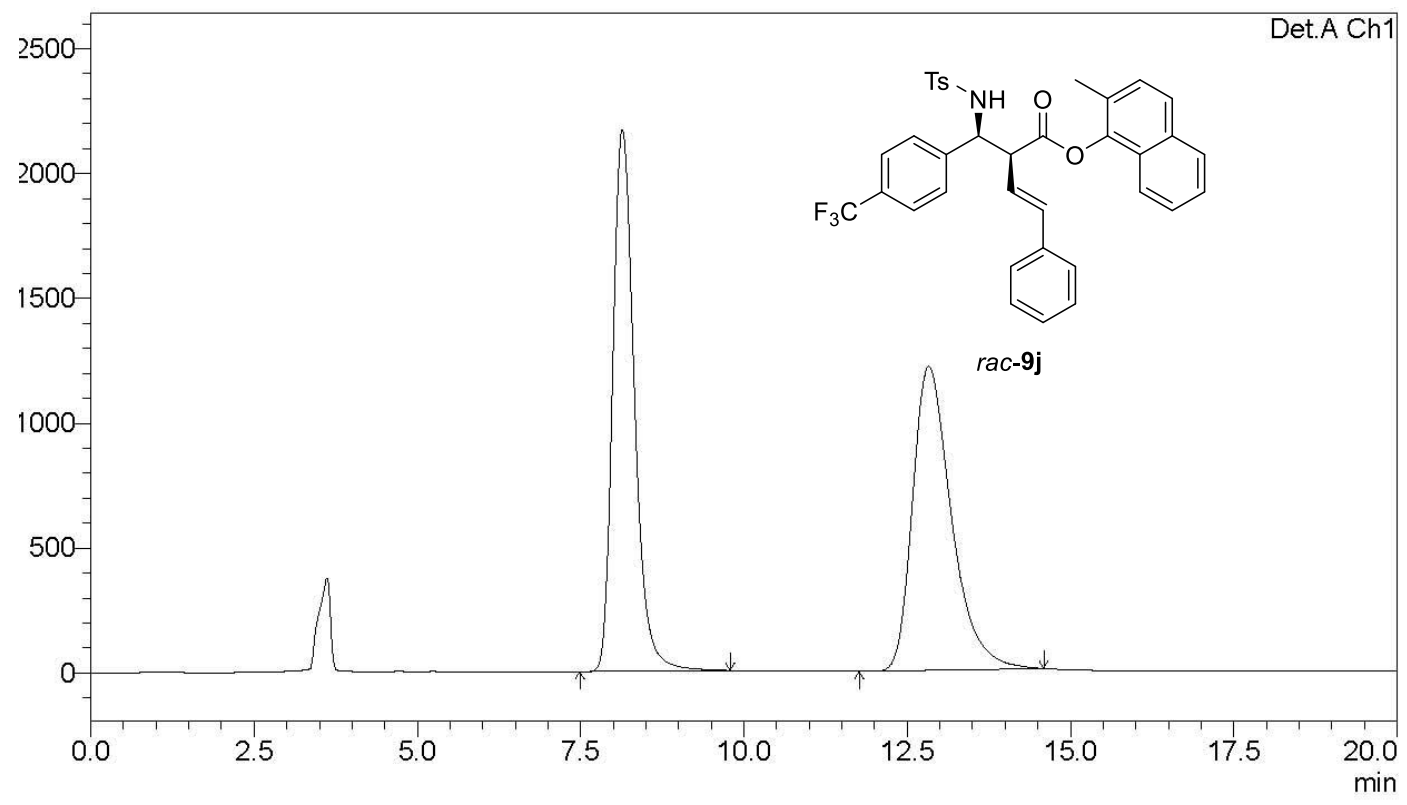

PeakTable

Detector A Ch1 220nm
\begin{tabular}{|r|r|r|r|r|r|}
\hline \multicolumn{1}{|c|}{ Peak\# } & Ret. Time & \multicolumn{1}{c|}{ Area } & Height & \multicolumn{1}{c|}{ Area \% } & \multicolumn{1}{|c|}{ Height \% } \\
\hline 1 & 8.135 & 49873841 & 2167924 & 49.854 & 64.039 \\
\hline 2 & 12.824 & 50166060 & 1217374 & 50.146 & 35.961 \\
\hline Total & & 100039900 & 3385298 & 100.000 & 100.000 \\
\hline
\end{tabular}

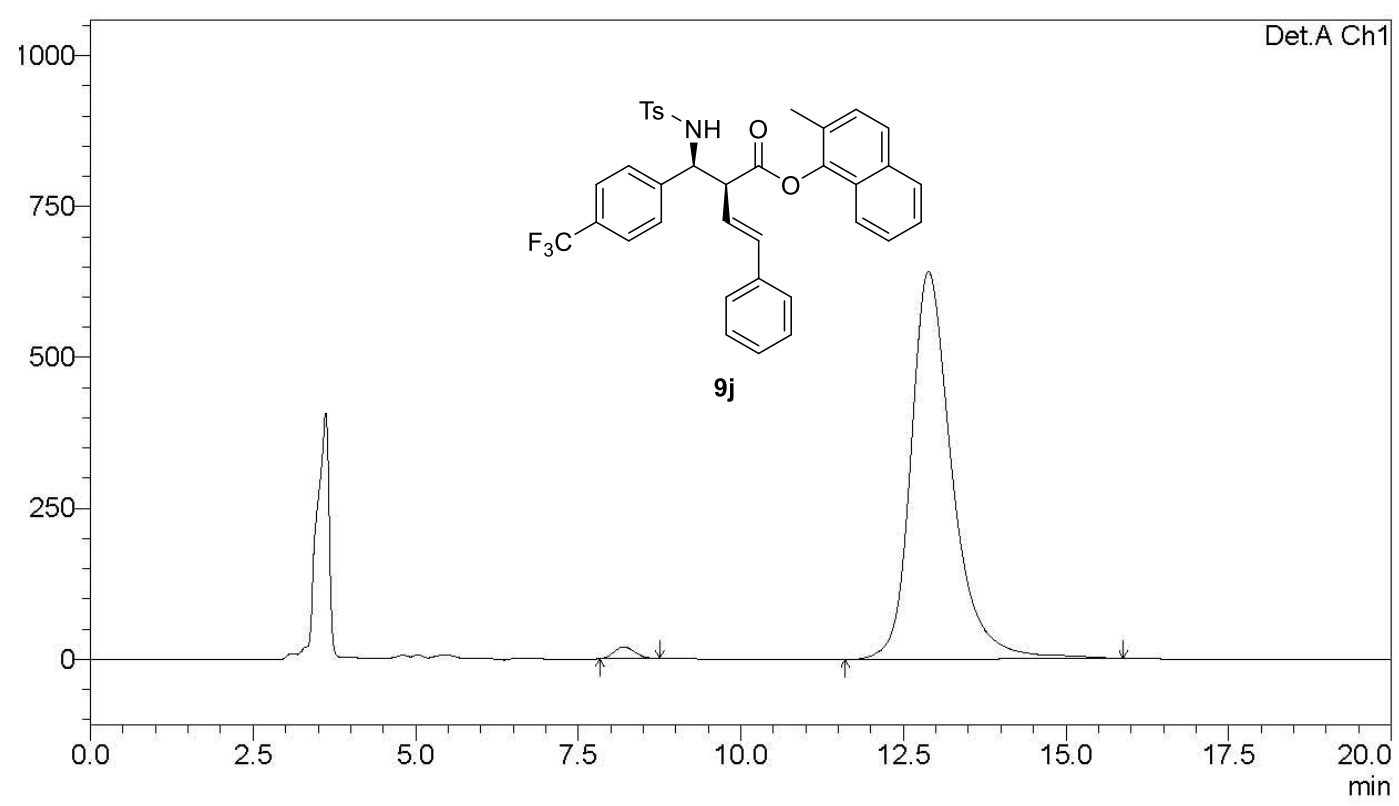

Detector A Chl $220 \mathrm{~nm}$
\begin{tabular}{|r|r|r|r|r|r|}
\hline \multicolumn{1}{|c|}{ Peak\# } & Ret. Time & Area & Height & Area \% & Height \% \\
\hline 1 & 8.199 & 409827 & 19332 & 1.436 & 2.927 \\
\hline 2 & 12.883 & 28137346 & 641104 & 98.564 & 97.073 \\
\hline Total & & 28547173 & 660436 & 100.000 & 100.000 \\
\hline
\end{tabular}




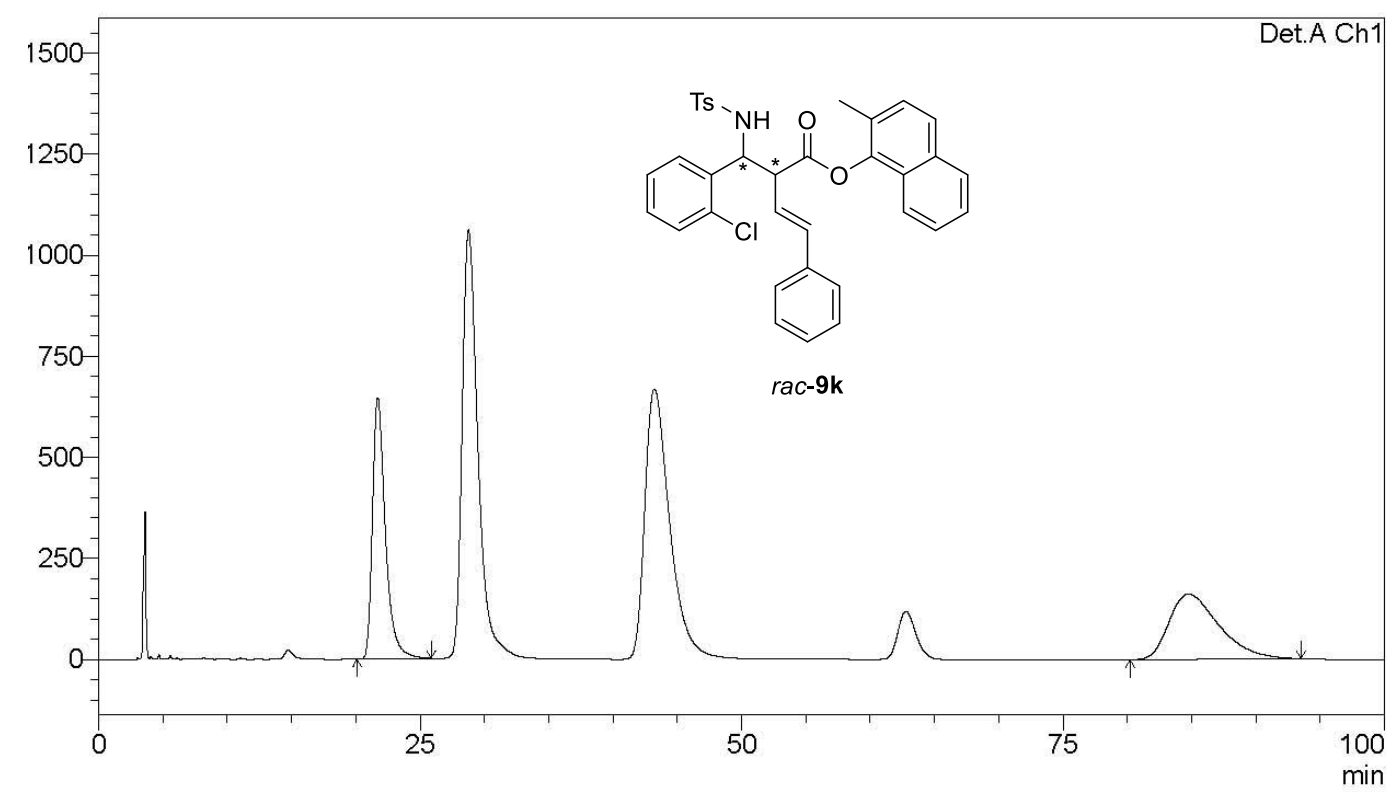

Detector A Ch1 220nm
\begin{tabular}{|r|r|r|r|r|r|}
\hline Peak\# & Ret. Time & \multicolumn{1}{|c|}{ Area } & Height & Area \% & \multicolumn{1}{c|}{ Height \% } \\
\hline 1 & 21.703 & 44955900 & 646423 & 50.697 & 79.995 \\
\hline 2 & 84.751 & 43720160 & 161660 & 49.303 & 20.005 \\
\hline Total & & 88676060 & 808083 & 100.000 & 100.000 \\
\hline
\end{tabular}

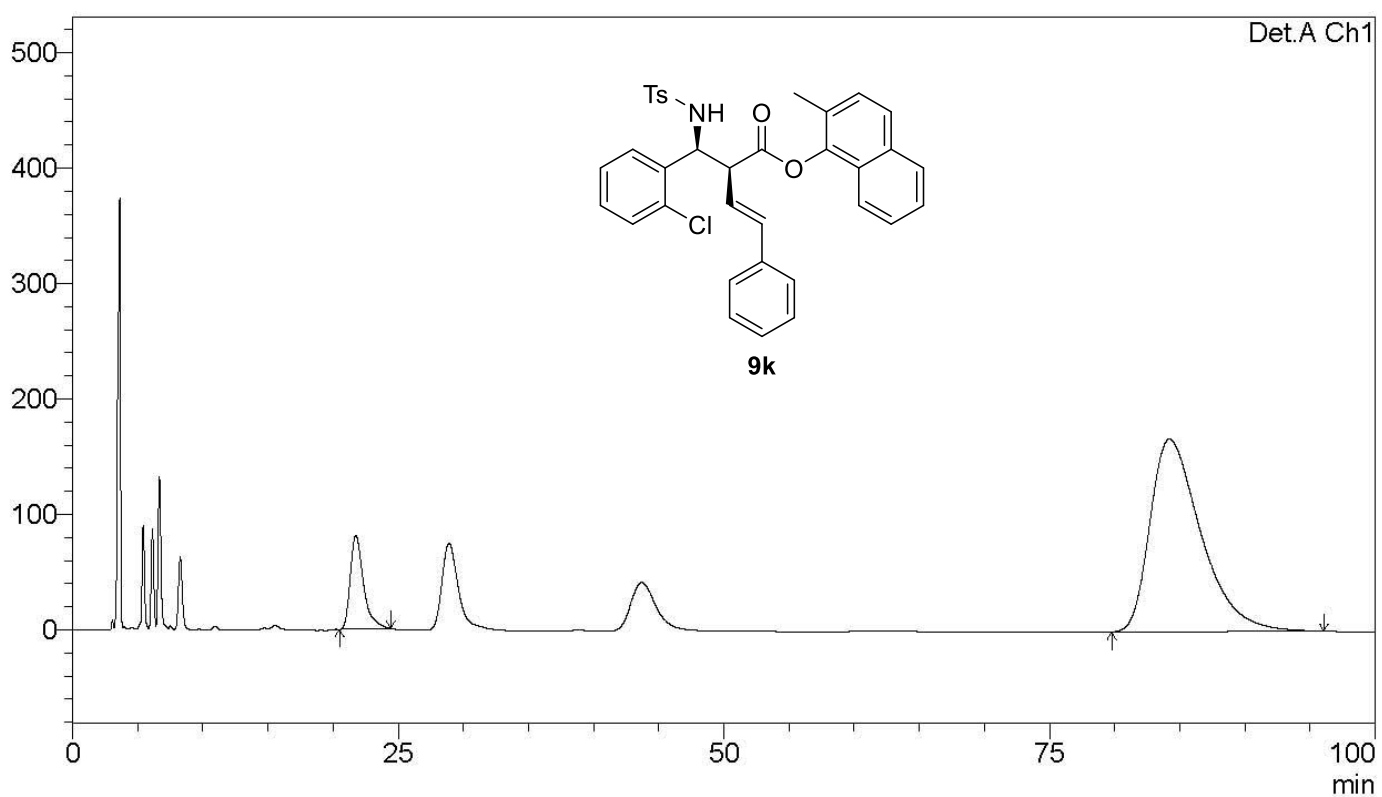

PeakTable

Detector A Ch1 220nm
\begin{tabular}{|r|r|r|r|r|r|}
\hline Peak\# & Ret. Time & \multicolumn{1}{|c|}{ Area } & Height & Area $\%$ & \multicolumn{1}{|c|}{ Height \% } \\
\hline 1 & 21.742 & 5718777 & 80894 & 11.194 & 32.647 \\
\hline 2 & 84.218 & 45368288 & 166890 & 88.806 & 67.353 \\
\hline Total & & 51087065 & 247783 & 100.000 & 100.000 \\
\hline
\end{tabular}




\section{Area \% Report}

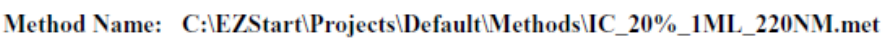

Data: $\quad$ C:IEZStart $\quad$ Projects $\backslash$ Default $\backslash$ Data\BLAN1111122

User: System

Acquired: $\quad 5 / 1 / 2015$ 11:37:38 AM

Printed: $\quad$ 5/1/2015 12:38:36 PM
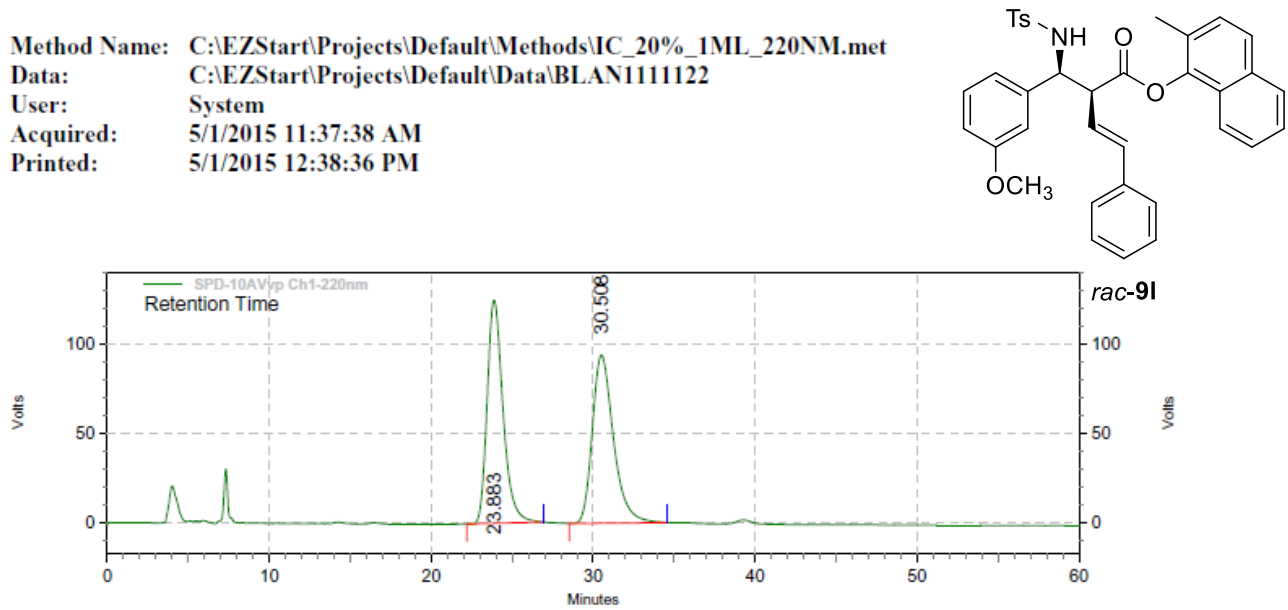

SPD-10AVvp

Ch1-220nm

Results

Retention Time

\begin{tabular}{rrrrr} 
& Area & Area \% & Height & Height \% \\
\hline 23.883 & 8496192 & 49.85 & 124680 & 57.03 \\
30.508 & 8545650 & 50.15 & 93960 & 42.97
\end{tabular}

Totals

17041842

100.00

218640

100.00

\section{Area \% Report}

Method Name: C: $\backslash$ EZStart $\backslash$ Projects $\backslash$ Default $\backslash$ Methods $\backslash$ IC 20\% 1ML 220NM.met

Data: $\quad$ C: $\backslash$ EZStart $\backslash$ Projects $\backslash$ Default $\backslash$ Data $\backslash$ BLAN11111222

User: System

Acquired: $\quad$ 5/1/2015 12:41:02 PM

Printed: $\quad$ 5/1/2015 1:43:07 PM

Page 1 of 1

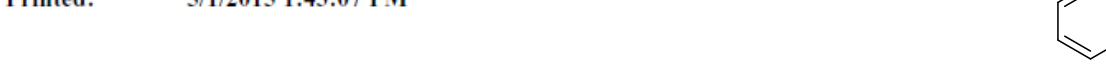
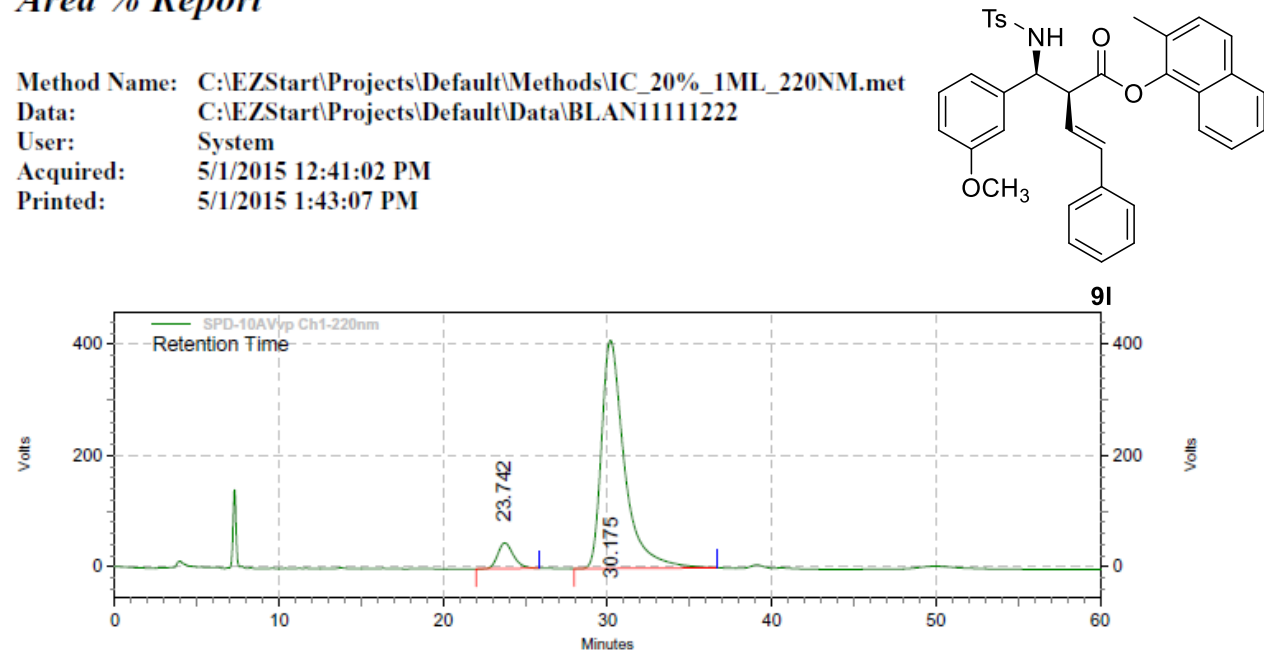

SPD-10AVvp

Ch1-220nm

Results

Retention Time

\begin{tabular}{rrr}
7.14 & Height & Height $\%$ \\
\hline & 46223 & 10.12
\end{tabular}

30.175

39991674

410398

89.88

Totals

43065602

100.00

456621

100.00 
$====$ Shimadzu LCsolution LC Data Image $====$ <Chromatogram>

$\mathrm{mV}$

C: ILabSolutionsIDataIProject1YI-SKR-18

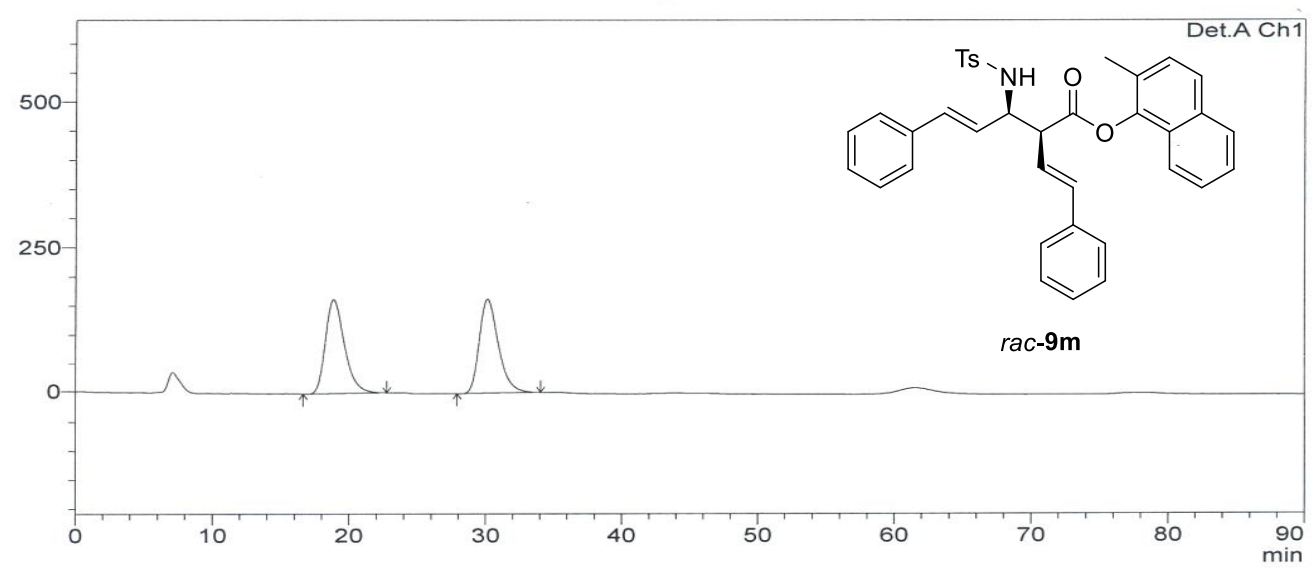

PeakTable

Detector A Ch1 $220 \mathrm{~nm}$
\begin{tabular}{|r|r|r|r|r|r|}
\hline Peak\# & Ret. Time & Arrea & Height & Area \% & \multicolumn{1}{|c|}{ Height \% } \\
\hline 1 & 20.500 & 19624462 & 305194 & 50.144 & 51.188 \\
\hline 2 & 30.108 & 18746518 & 290444 & 49.856 & 48.812 \\
\hline Total & & 38370980 & 59563.8 & 100.000 & 100.000 \\
\hline
\end{tabular}

$====$ Shimadzu LCsolution LC Data Image $====$

<Chromatogram>

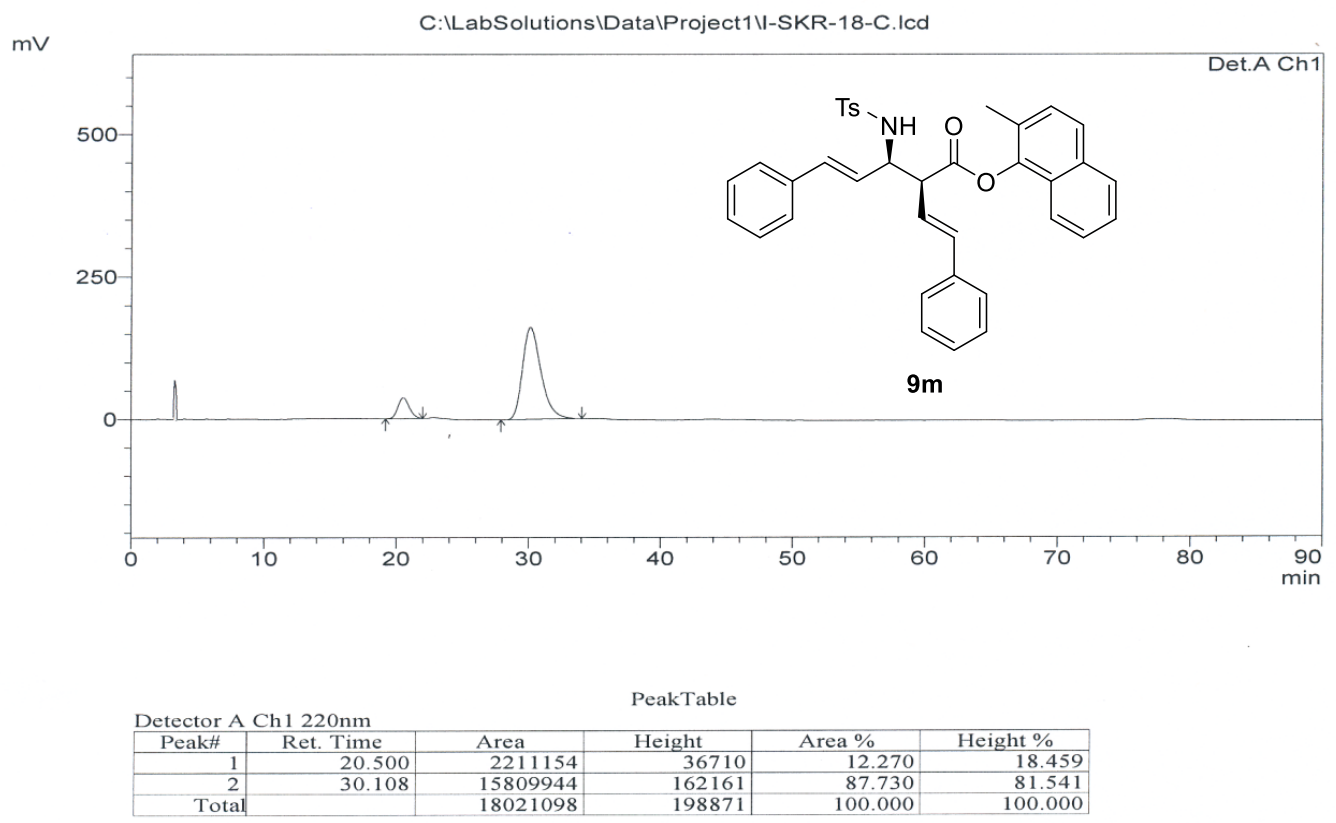




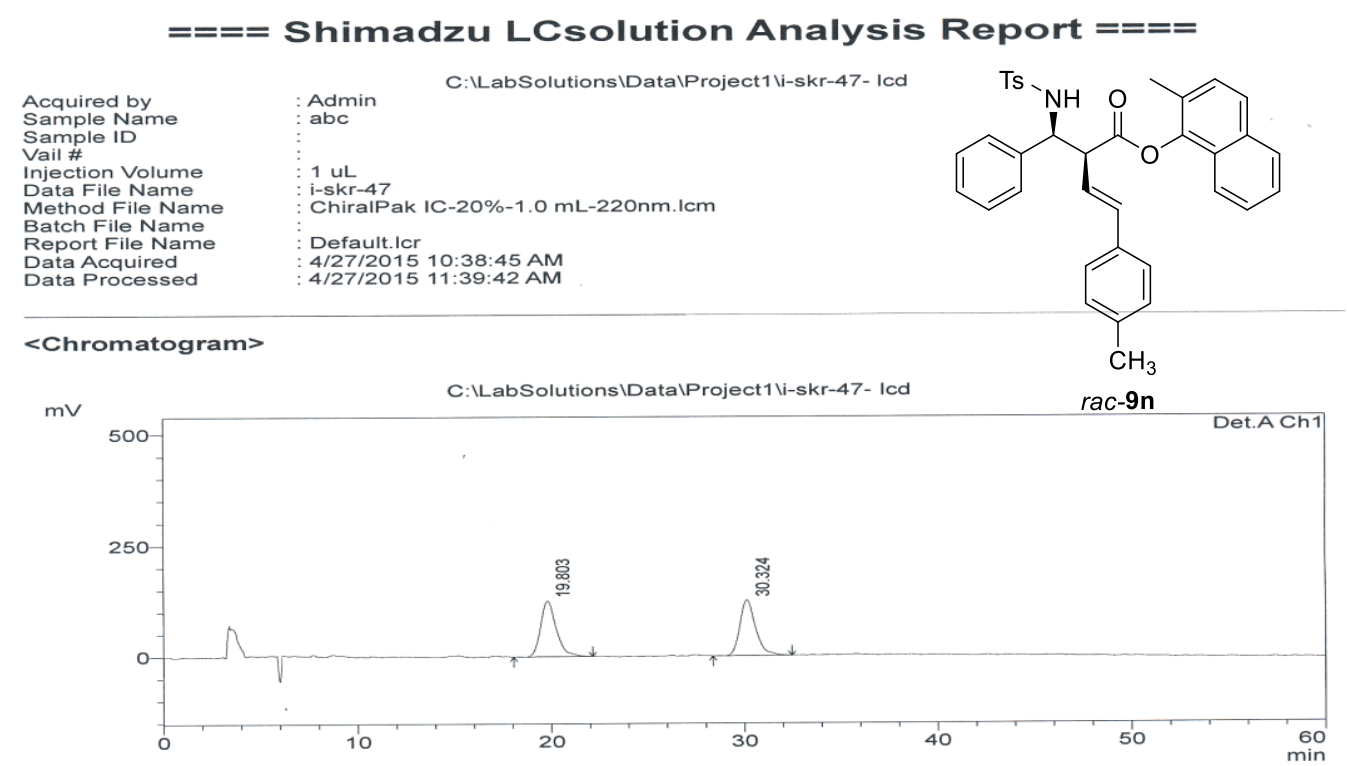

1 Det.A Ch1/220nm

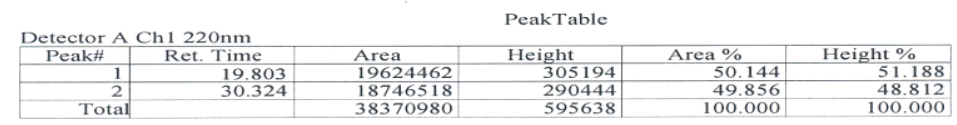

$====$ Shimadzu LCsolution Analysis Report $===$

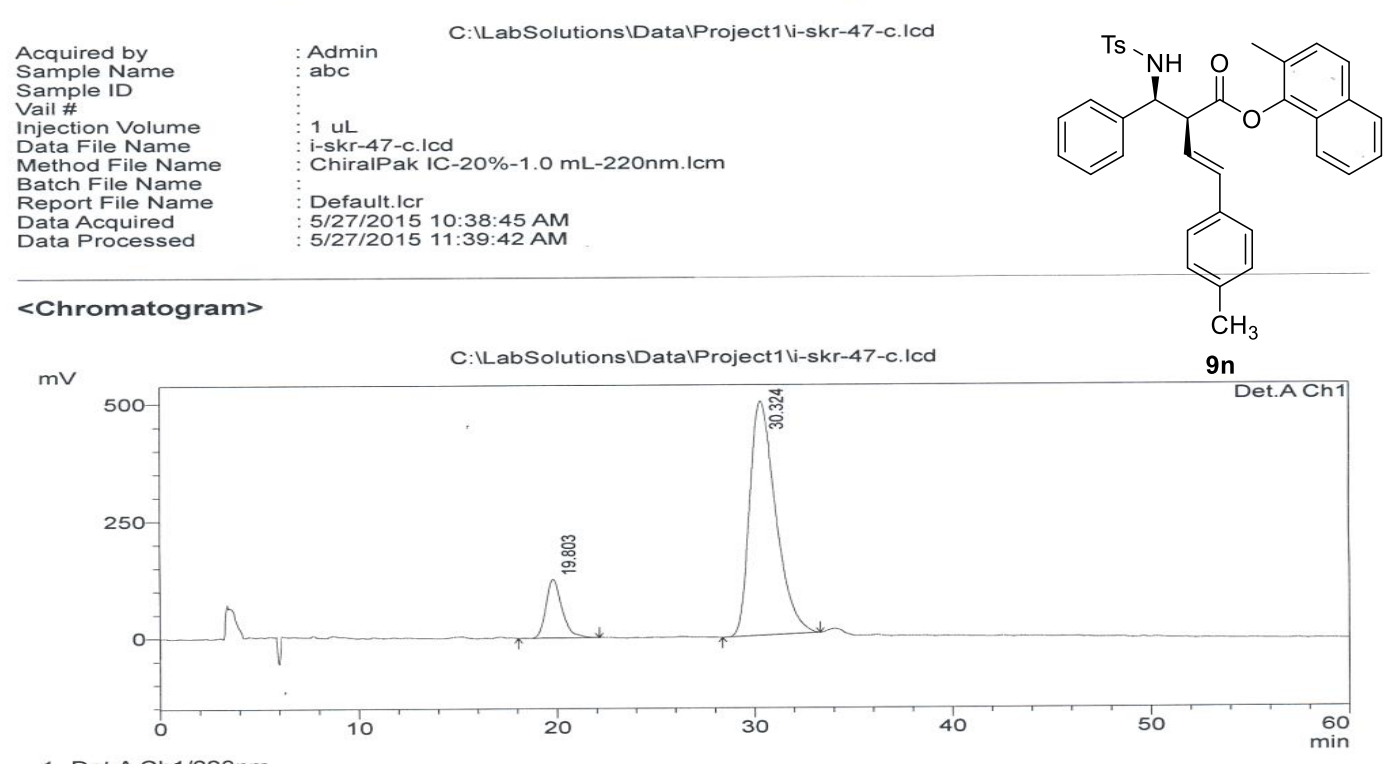

1 Det.ACh1/220nm

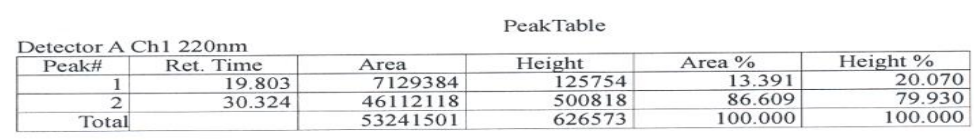


Method Name: C:\EZStart $\backslash P$ Projects $\backslash$ Default $\backslash$ Methods $\backslash C$ IC $0 \%$ 1ML_220NM.-60-met.met

Data: $\quad C: \backslash E Z S t a r t \backslash P r o j e c t s \backslash D e f a u I t \backslash D a t a \backslash I-S K R-50-R A$

User: $\quad$ System

Acquired: $\quad$ 5/1/2015 4:58:46 PM

Printed: $\quad$ 5/1/2015 5:35:26 PM

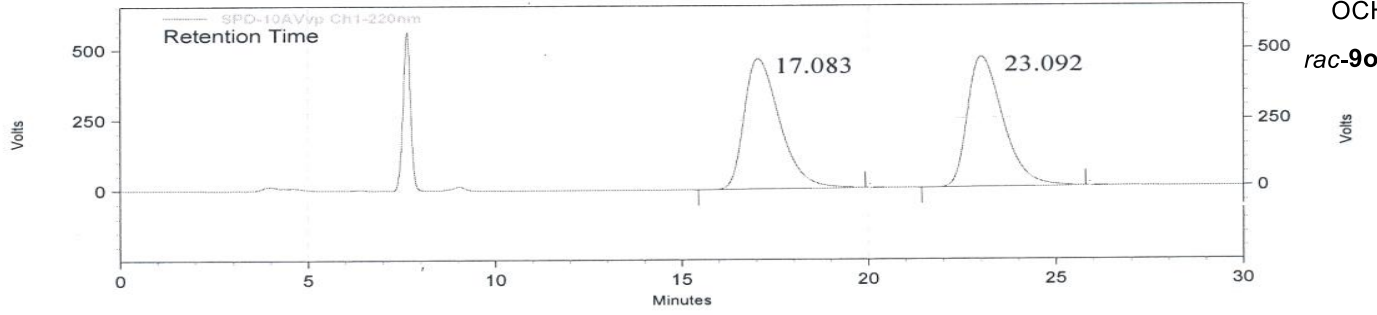

SPD-10AVVP

Ch1-220nm

Results

\begin{tabular}{rrrrrr}
$\begin{array}{c}\text { Retention Time } \\
\text { Retrea }\end{array}$ & Area & Height & Height \% \\
\hline 17.083 & 2292235 & 50.41 & 47744 & 59.69 \\
23.092 & 2255187 & 49.59 & 32244 & 40.31 \\
\hline \multicolumn{2}{|c|}{ Totals } & 4547422 & 100.00 & 79988 & 100.00 \\
\hline
\end{tabular}

\section{Area \% Report}

Method Name: C: $\backslash$ EZStart $\backslash$ Projects $\backslash$ Default $\backslash$ Methods $\backslash$ IC_20\%_1ML_220NM.-60-met.met

Data:

User: C: $\backslash$ EZStart $\backslash$ Projects $\backslash$ Default $\backslash D a t a \backslash I-S K R-50$

Printed:
Page 1 of 1

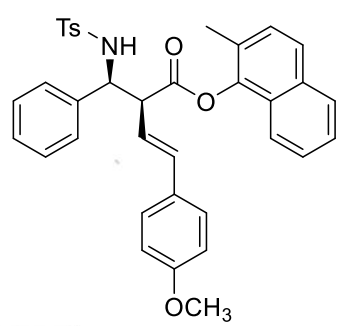

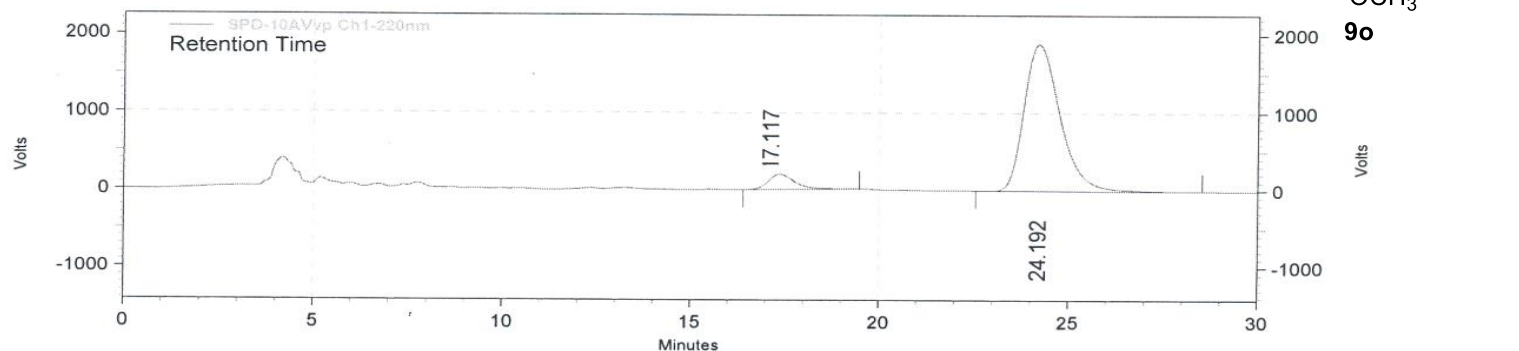

SPD-10AVvp

Ch1-220nm

Results

\begin{tabular}{rrrrrr|}
$\begin{array}{r}\text { Retention Time } \\
\text { R.117 }\end{array}$ & Area & Area \% & Height & Height \% \\
\hline 24.192 & 3073928 & 7.14 & 46223 & 10.12 \\
\multicolumn{2}{|c|}{39991674} & 92.86 & 410398 & 89.88 \\
\hline Totals & 43065602 & 100.00 & 456621 & 100.00 \\
\hline
\end{tabular}




\section{Area \% Report}

Method Name: C: $\backslash$ EZStart $\backslash P$ Projects $\backslash$ Default $\backslash$ Methods $\backslash C$ IC 20\%_1ML_220NM.met

Data: C: $\backslash$ EZStart $\backslash$ Projects $\backslash$ Default $\backslash$ Data $\backslash$ BLAN11111

User: System

Acquired: $\quad 3 / 1 / 2015$ 10:12:51 AM

Printed: $\quad 3 / 1 / 2015$ 11:23:39 AM

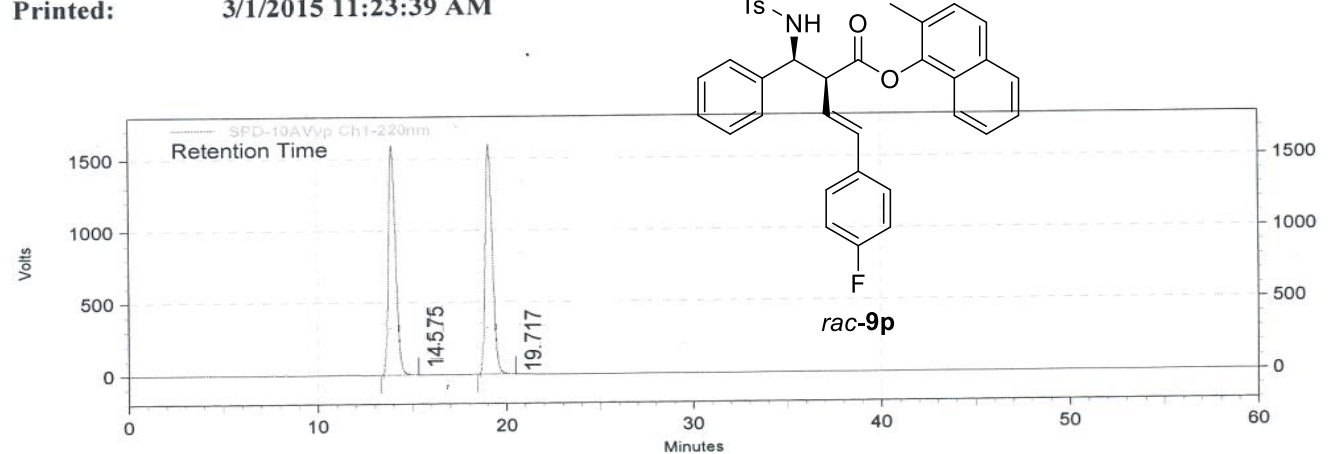

SPD-10AVvp

Ch1-220nm

Results

\begin{tabular}{rrrrrr}
$\begin{array}{c}\text { Retention Time } \\
\text { Reten }\end{array}$ & Area \% & Height & Height \% \\
\hline 14.575 & 22105161 & 50.72 & 599274 & 59.50 \\
19.917 & 21480541 & 49.28 & 407925 & 40.50 \\
\hline Totals & 43585702 & 100.00 & 1007199 & 100.00 \\
\hline
\end{tabular}

Method Name: C: $\backslash$ EZStart $\backslash$ Projects $\backslash$ Default $\backslash$ Methods $\backslash$ IC_20\%_1ML_220NM.met

Data: $\quad C: \backslash$ EZStart $\backslash$ Projects $\backslash$ Default|Data $\backslash$ BLAN11111

User: System

Acquired: $\quad$ 5/1/2015 10:12:51 AM

Printed: $\quad$ 5/1/2015 11:23:39 AM

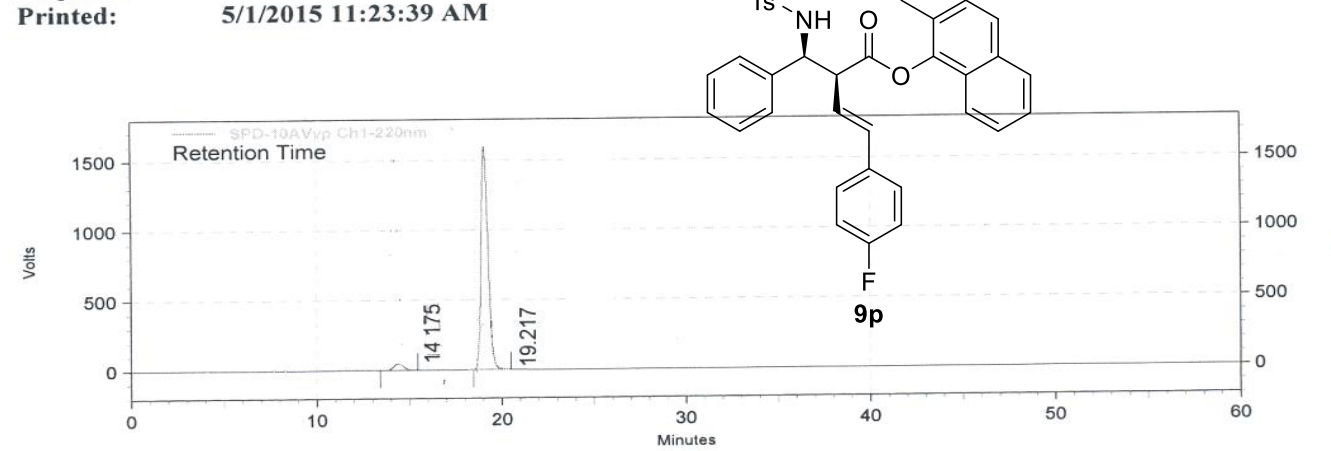

SPD-10AVvp

Ch1-254nm

Results

\begin{tabular}{r|rrrrr|}
$\begin{array}{c}\text { Results } \\
\text { Retention Time }\end{array}$ & Area & Area \% & Height & Height \% \\
\hline 14.175 & 1656378 & 3.81 & 46267 & 2.82 \\
19.217 & 41763616 & 96.19 & 1593176 & 97.18 \\
\hline Totals & 43419994 & 100.00 & 1639443 & 100.00 \\
\hline
\end{tabular}


$<$ Chromatogram $>$

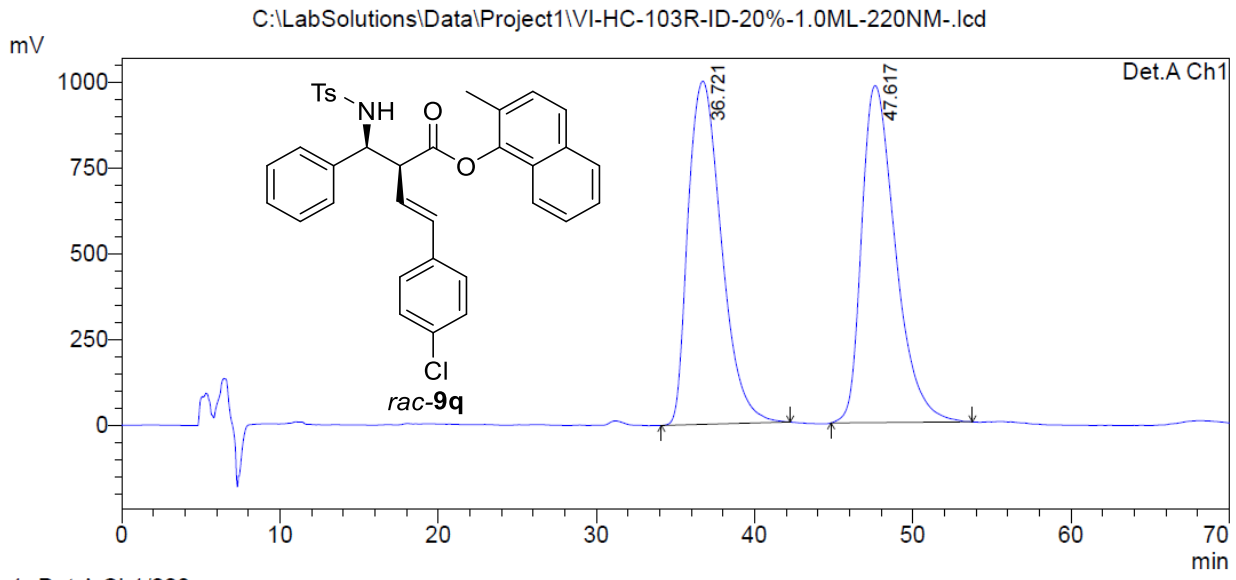

1 Det.A Ch1/220nm

Detector A Ch1 $220 \mathrm{~nm}$
\begin{tabular}{|r|r|r|r|r|r|}
\hline Peak\# & Ret. Time & Area & Height & Area $\%$ & Height $\%$ \\
\hline 1 & 36.721 & 146549089 & 1000845 & 50.057 & 50.450 \\
\hline 2 & 47.617 & 146216494 & 982990 & 49.943 & 49.550 \\
\hline Total & & 292765583 & 1983835 & 100.000 & 100.000 \\
\hline
\end{tabular}

\section{$<$ Chromatogram>}

$\mathrm{mV}$

C:LabSolutions IDatalProject1IVI-HC-103-ID-20\%-1.0ML-220NM.Icd

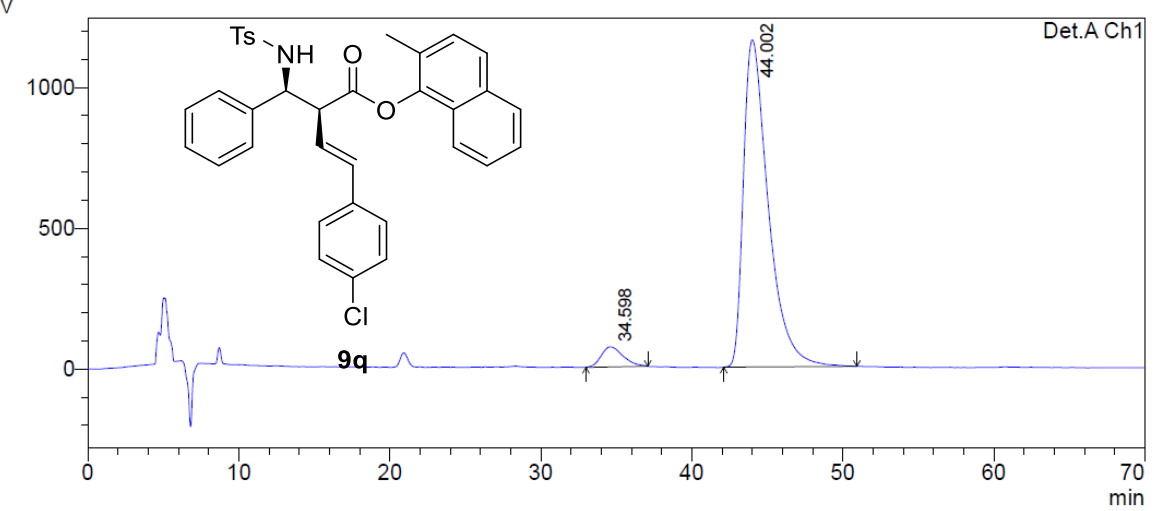

1 Det.A Ch1/220nm

Detector A Ch1 220nm
\begin{tabular}{|r|r|r|r|r|r|}
\hline Peak\# & Ret. Time & \multicolumn{1}{c|}{ Area } & Height & Area $\%$ & \multicolumn{1}{c|}{ Height $\%$} \\
\hline 1 & 34.598 & 7215883 & 71289 & 5.146 & 5.783 \\
\hline 2 & 44.002 & 133020195 & 1161378 & 94.854 & 94.217 \\
\hline Total & & 140236079 & 1232667 & 100.000 & 100.000 \\
\hline
\end{tabular}




\section{<Chromatogram>}

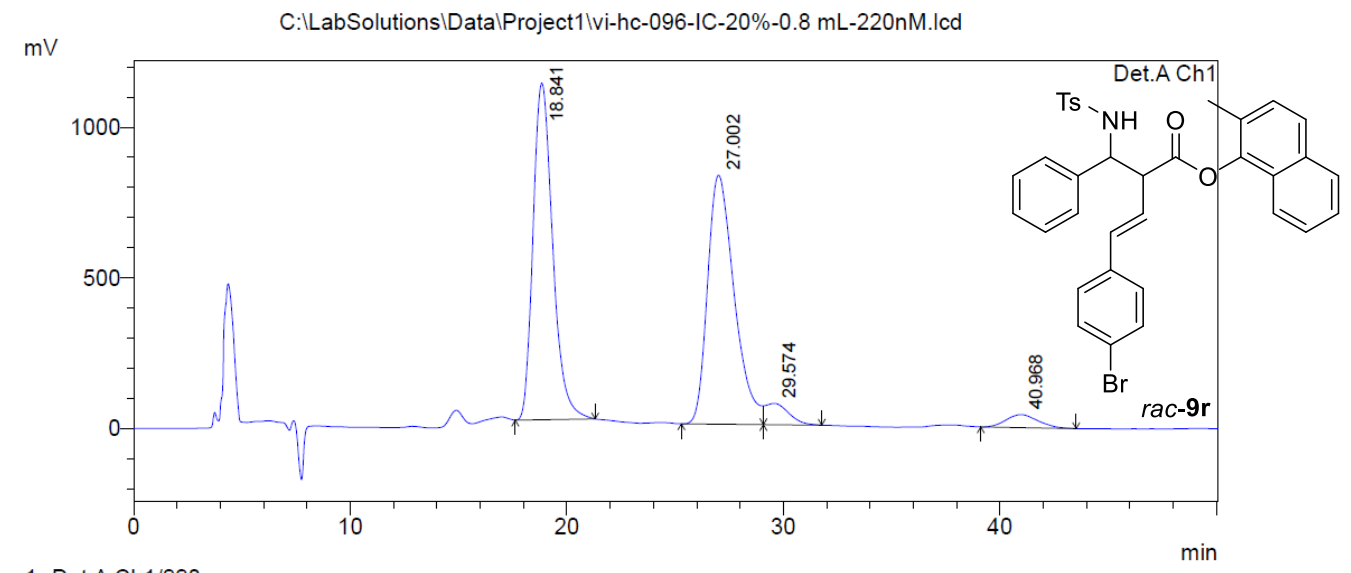

1 Det.A Ch1/220nm

Detector A Ch1 220nm
\begin{tabular}{|r|r|r|r|r|r|}
\hline \multicolumn{1}{|r|}{ Peak\# } & Ret. Time & \multicolumn{1}{c|}{ Area } & \multicolumn{1}{c|}{ Height } & Area $\%$ & \multicolumn{1}{c|}{ Height $\%$} \\
\hline 1 & 18.841 & 70421010 & 1118163 & 46.546 & 54.303 \\
\hline 2 & 27.002 & 71061113 & 826598 & 46.969 & 40.143 \\
\hline 3 & 29.574 & 5359077 & 71081 & 3.542 & 3.452 \\
\hline 4 & 40.968 & 4452862 & 43292 & 2.943 & 2.102 \\
\hline Total & & 151294062 & 2059134 & 100.000 & 100.000 \\
\hline
\end{tabular}

\section{<Chromatogram>}

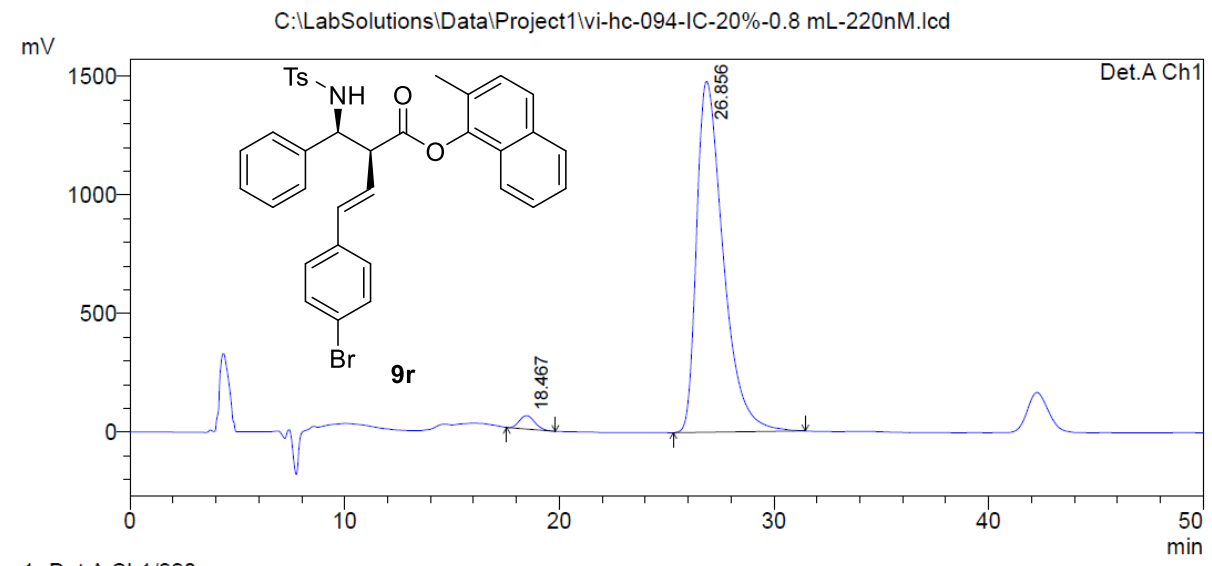

1 Det.A Ch1/220nm

PeakTable

Detector A Ch1 220nm
\begin{tabular}{|r|r|r|r|r|r|}
\hline Peak\# & Ret. Time & \multicolumn{1}{c|}{ Area } & Height & Area $\%$ & \multicolumn{1}{c|}{ Height $\%$} \\
\hline 1 & 18.467 & 2844127 & 56686 & 2.178 & 3.691 \\
\hline 2 & 26.856 & 127736176 & 1479285 & 97.822 & 96.309 \\
\hline Total & & 130580303 & 1535971 & 100.000 & 100.000 \\
\hline
\end{tabular}


$\mathrm{mV}$

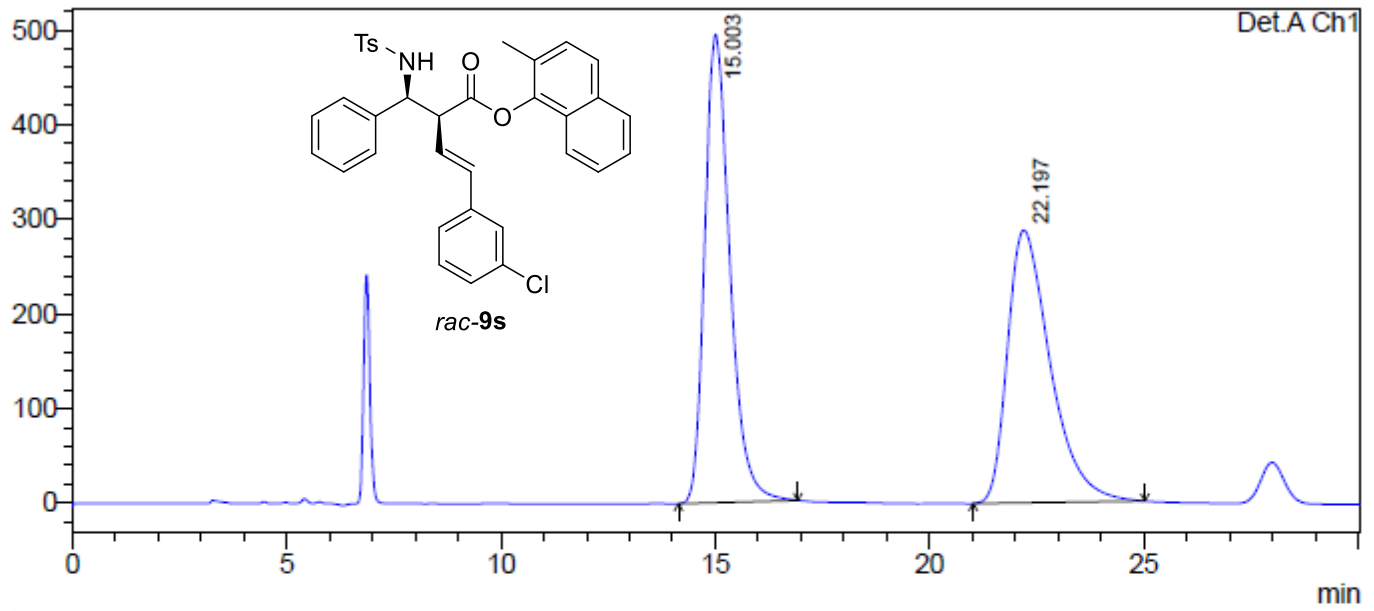

1 Det.A Ch1/254nm

PeakTable

Detector A Ch1 $254 \mathrm{~nm}$
\begin{tabular}{|r|r|r|r|r|r|}
\hline Peak\# & Ret. Time & Area & Height & Area $\%$ & Height \% \\
\hline 1 & 15.003 & 20227980 & 494486 & 50.250 & 63.203 \\
\hline 2 & 22.197 & 20026538 & 287886 & 49.750 & 36.797 \\
\hline Total & & 40254518 & 782372 & 100.000 & 100.000 \\
\hline
\end{tabular}

$\mathrm{mV}$

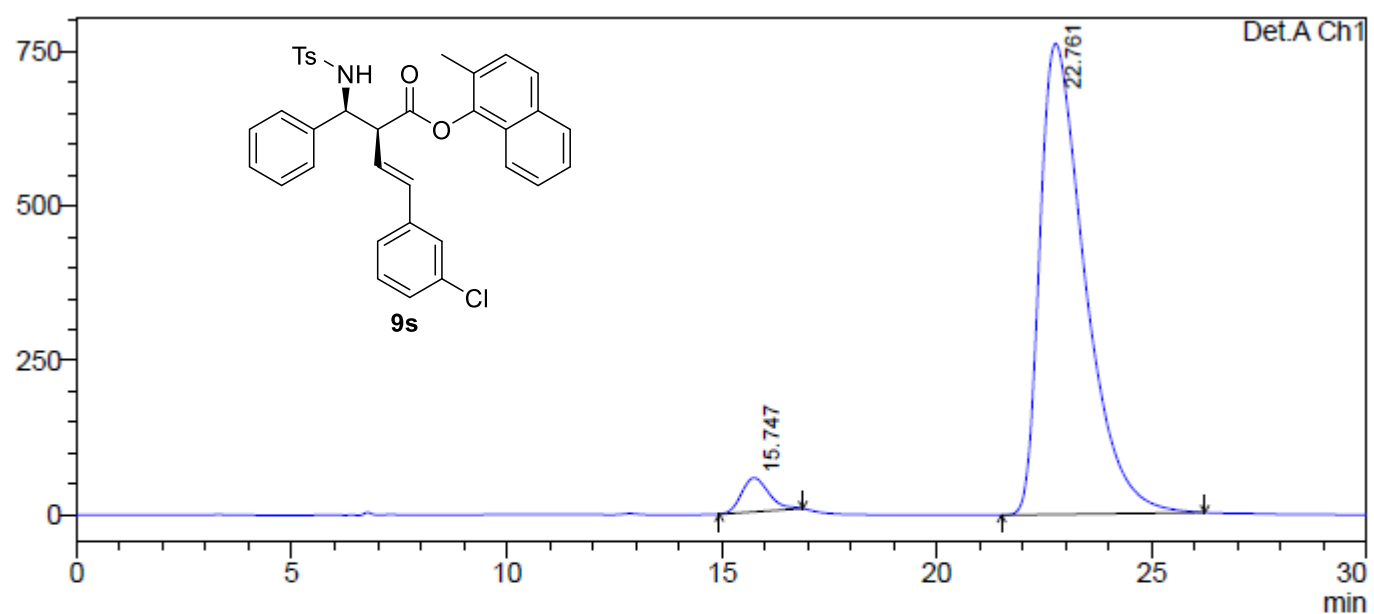

1 Det.A Ch1/254nm

PeakTable

Detector A Ch1 $254 \mathrm{~nm}$
\begin{tabular}{|r|r|r|r|r|r|}
\hline Peak\# & Ret. Time & \multicolumn{1}{|c|}{ Area } & Height & Area \% & \multicolumn{1}{c|}{ Height \% } \\
\hline 1 & 15.747 & 2414512 & 54875 & 4.178 & 6.721 \\
\hline 2 & 22.761 & 55383152 & 761648 & 95.822 & 93.279 \\
\hline Total & & 57797665 & 816523 & 100.000 & 100.000 \\
\hline
\end{tabular}




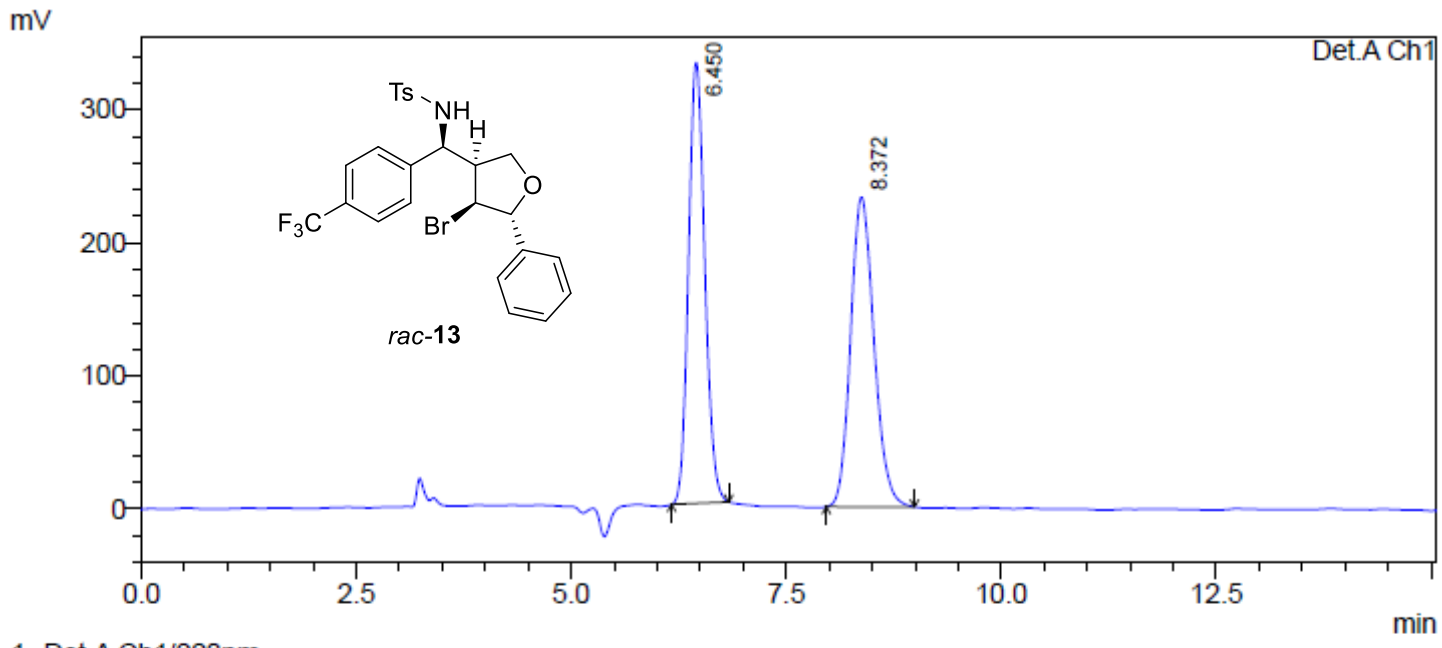

1 Det.A Ch $1 / 220 \mathrm{~nm}$

Detector A Ch1 220nm
\begin{tabular}{|r|r|r|r|r|r|}
\hline \multicolumn{1}{|c|}{ Peak\# $\#$} & Ret. Time & Area & Height & Area \% & Height \% \\
\hline 1 & 6.450 & 4366936 & 331105 & 49.750 & 58.724 \\
\hline 2 & 8.372 & 4410817 & 232728 & 50.250 & 41.276 \\
\hline Total & & 8777753 & 563832 & 100.000 & 100.000 \\
\hline
\end{tabular}

$\mathrm{mV}$

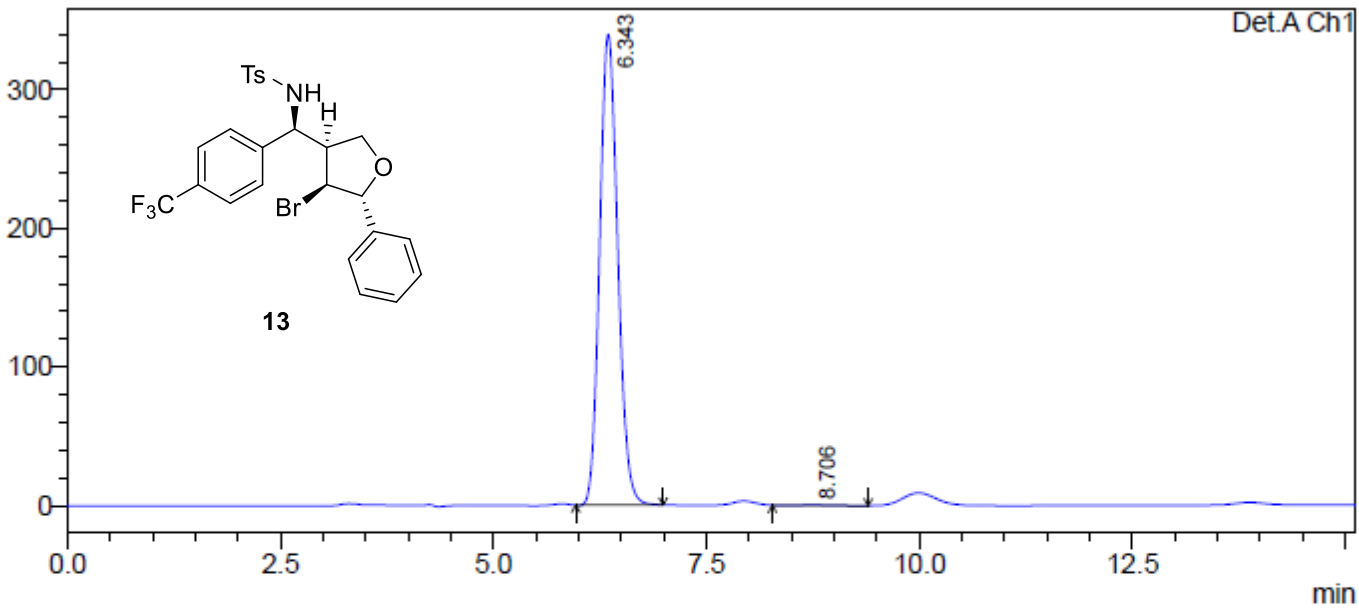

1 Det.A Ch1/254nm

PeakTable

Detector A Ch1 $254 \mathrm{~nm}$
\begin{tabular}{|r|r|r|r|r|r|}
\hline Peak\# & Ret. Time & \multicolumn{1}{|c|}{ Area } & \multicolumn{1}{c|}{ Height } & Area \% & \multicolumn{1}{c|}{ Height \% } \\
\hline 1 & 6.343 & 5019279 & 339394 & 99.957 & 99.925 \\
\hline 2 & 8.706 & 2155 & 256 & 0.043 & 0.075 \\
\hline Total & & 5021434 & 339650 & 100.000 & 100.000 \\
\hline
\end{tabular}

